\title{
Radiation driven winds of hot luminous stars
}

\section{Line statistics and radiative driving}

\author{
J. Puls, U. Springmann, and M. Lennon \\ Universitäts-Sternwarte München, Scheinerstr. 1, D-81679 München, Germany
}

Received March 18; accepted September 28, 1999

\begin{abstract}
This paper analyzes the inter-relation between line-statistics and radiative driving in massive stars with winds (excluding Wolf-Rayets) and provides insight into the qualitative behaviour of the well-known force-multiplier parameters $k_{\mathrm{CAK}}, \alpha$ and $\delta$, with special emphasis on $\alpha$.

After recapitulating some basic properties of radiative line driving, the correspondence of the local exponent of (almost) arbitrary line-strength distribution functions and $\alpha$, which is the ratio of optically thick to total line-force, is discussed. Both quantities are found to be roughly equal as long as the local exponent is not too steep.

We compare the (conventional) parameterization applied in this paper with the so-called $\bar{Q}$-formalism introduced by Gayley (1995) and conclude that the latter can be applied alternatively in its most general form. Its "strongest form", however (requiring the Ansatz $\bar{Q}=Q_{\text {o }}$ to be valid, with $Q_{\mathrm{o}}$ the line-strength of the strongest line), is justified only under specific conditions, typically for Supergiants with $T_{\text {eff }} \gtrsim 35000 \mathrm{~K}$.

The central part of this paper considers the question concerning the shape of the line-strength distribution function, with line-strength $k_{\mathrm{L}}$ as approximate depth independent ratio of line and Thomson opacity. Since $k_{\mathrm{L}}$ depends on the product of oscillator strength, excitationand ionization fraction as well as on elemental abundance, all of these factors have their own, specific influence on the final result.
\end{abstract}

At first, we investigate the case of hydrogenic ions, which can be treated analytically. We find that the exponent of the differential distribution is $-4 / 3$ corresponding to $\alpha=2 / 3$, as consequence of the underlying oscillator strength distribution. Furthermore, it is shown that for trace ions one stage below the major one (e.g., Hi in hot winds) the equality $\alpha+\delta \approx 1$ is valid throughout the wind.

For the majority of non-hydrogenic ions, we follow the statistical approach suggested by Allen (1966), refined in

Send offprint requests to: J. Puls

e-mail: uh101aw@usm.uni-muenchen.de a number of ways which allow, as a useful by-product, the validity of the underlying data bases to be checked. Per ion, it turns out that the typical line-strength distribution consists of two parts, where the first, steeper one is dominated by excitation effects and the second one follows the oscillator strength distribution of the specific ion.

By summing up the contributions of all participating ions, this direct influence of the oscillator strength distribution almost vanishes. It turns out, however, that there is a second, indirect influence controlling the absolute line numbers and thus $k_{\mathrm{CAK}}$. From the actual numbers, we find an average exponent of order $-1.2 \ldots-1.3$, similar to the value for hydrogen.

Most important for the shape of the total distribution is the difference in line-statistics between iron group and light ions as well as their different (mean) abundance. Since the former group comprises a large number of meta-stable levels, the line number from iron group elements is much higher, especially at intermediate and weak line-strengths. Additionally, this number increases significantly with decreasing temperature (more lines from lower ionization stages). In contrast, the line-strength distribution of light ions remains rather constant as function of temperature.

Since the line-strength depends linearly on the elemental abundance, this quantity controls the relative influence of the specific distributions on the total one and the overall shape. For solar composition, a much more constant slope is found, compared to the case if all abundances were equal.

In result, we find (for solar abundances) that iron group elements dominate the distribution at low and intermediate values of line-strength (corresponding to the acceleration in the inner wind part), whereas light ions (including hydrogen under A-star conditions) dominate the high $k_{\mathrm{L}}$ end (outer wind). Typically, this part of the distribution is steeper than the rest, due to excitation effects. 
Finally, the influence of global metallicity $z$ is discussed. We extend already known scaling relations (regarding mass-loss, terminal velocity and wind-momentum rate) with respect to this quantity. In particular, we demonstrate that, besides the well-known direct effect $\left(k_{\mathrm{CAK}} \propto z^{1-\alpha}\right)$, the curvature of the line-strength distribution at its upper end induces a decrease of $\alpha$ for low metallicity and/or low wind density.

Summarizing the different processes investigated, the force-multiplier parameter $\alpha$ becomes a decreasing function of decreasing $T_{\text {eff }}$, increasing $k_{1}=\mathrm{d} v / \mathrm{d} r / \rho$ and decreasing global metallicity $z$, consistent with the findings of earlier and present empirical results and observations.

Key words: atomic data - stars: atmospheres — stars: early type — stars: mass-loss

\section{Introduction}

Over the last three decades the idea of radiative driving by metal-line absorption/scattering has been applied with increasing success. Besides the original motivation of explaining the supersonic outflows of OB-star winds and predicting their strengths (Lucy \& Solomon 1970; Castor et al. 1975 (CAK)), a much wider domain of sophisticated physical processes is considered now. To name only a few recent and exciting developments, there is, e.g., the possibility of compressing wind material into the stellar equatorial zone and even creating a disk due to the combined effects of (radial) line and centrifugal force in case of large rotational rates (Bjorkman \& Cassinelli 1993) and the counteracting role of non-radial line-forces which might inhibit this process (Owocki et al. 1996). Another example (although originating from the very beginning: again Lucy \& Solomon 1970) would be the theory of line-force instability (Owocki \& Rybicki 1984, 1985) and its ongoing refinements (Owocki \& Puls 1996, 1999), which still awaits direct observational proof.

Going extragalactic, it turned out that the observed wind-momentum rate of supergiant winds allows for a determination of distances (Kudritzki et al. 1995) and may finally become an independent alternative to using Cepheids as distance indicators on the intermediate distance scale up to the Virgo Cluster. Even further out, the theory of radiative line driving and its possible instability is providing a first step towards explaining the physics of BAL-QSOs (Arav \& Li 1994; Arav et al. 1994; Feldmeier et al. 1997).

All of these investigations and theories have one thing in common: Although the specific line transfer for obtaining the radiation force per line is treated differently to meet the required physics, the total line force arising from thousands of contributing lines is calculated - either directly or indirectly - by means of a certain statistical description of the line distribution.

This procedure still follows the ingenious Ansatz by CAK and improvements obtained by Abbott (1982), which to its end requires the knowledge of only three "numbers" $k_{\mathrm{CAK}}, \alpha$ and $\delta$, the so-called force-multiplier parameters, in order to extrapolate from the behaviour of one line to the effects of the total line ensemble.

Unfortunately, the physical explanation of their origin and the discussion of their behaviour under various physical conditions is rather unsatisfactory. The only exception (to our knowledge) is the publication by Gayley (1995), which provides a profound insight into the efficiency of line driving (compared to electron scattering) and suggests a modified parameterization of the line-force, which however does not give any further clue concerning the involved line-statistics itself.

Nevertheless and especially in view of the wide use of the (improved) CAK- parameterization, the present situation is mostly unclear. This even more so, since it is the actual numerical value of one or more of the forcemultiplier parameters which may allow for certain effects to arise or rather to inhibit the process.

We want to mention here only two examples: The theoretical basis of the wind-momentum luminosity relation relies on the parameter $\alpha$ (actually $\alpha-\delta$ ) to be close to $2 / 3$, and a consistent calculation of a wind-compressed disk (neglecting non-radial forces) requires a (maybe too) large value of $\delta$.

Recently, Kudritzki et al. (1998) have presented a method and first results on how to obtain an improved parameterization of the line-force by allowing for a depth dependence of the force-multiplier parameters. However, also this paper is based on a purely descriptive approach, namely by calculating line-forces under various conditions and then by fitting the result to the modified parameterized expression. Although the final outcome of this project will prove to be useful for many applications, the question about the underlying physics still remains.

Since radiation driven wind theory has proven to work under different conditions and the physics of radiation driving is not only a black box process requiring the collection (or guess!) of various values for $k_{\mathrm{CAK}}, \alpha$ and $\delta$ in order to solve the hydro-equations, we feel that a more thorough investigation of the underlying statistics, physics and consequences has to be performed. The present paper is intended to answer at least some of the obvious questions and is organized as follows. In Sect. 2, we review the problem of how to calculate the line-force arising from an ensemble of lines by means of the so-called line-strength distribution function, where in a first step the "standard" representation is used. Additionally, however, we investigate also different distribution functions and interpret the ensemble line-force in a more general way. In Sect. 3, we turn to "reality" and present our method to derive NLTE 
line-distribution functions valid for the considered wind plasma conditions. We show typical examples and comment on Gayley's (1995) $\bar{Q}$ - formalism. In Sect. 4 , we try to understand the fundamental physics which controls the slope of the line-strength distribution function and hence the actual value of $\alpha$. Section 5 focuses on the consequences if the plasma conditions are changed, especially due to a different metallicity and/or mean wind density, and Sect. 6 summarizes our results and gives some caveats regarding the limits of our investigation.

\section{The line-force from an ensemble of lines and the line-strength distribution function}

In this section, we review the basic physics of radiative driving of an ensemble of lines and interpret the final outcome in terms of a so-called "line-strength distribution function". To this end, we allow for a number of approximations which will turn out to be of either minor importance or will be relaxed during further proceedings. Specifically,

- we will neglect the finite-disk effect and consider only radially streaming photons. The corresponding modification to the total line-force (cf. Friend \& Abbott 1986 and Pauldrach; Puls \& Kudritzki 1986) can be easily incorporated afterwards;

- We will use the Sobolev approximation for calculating the line-force of a single line, neglecting line-overlap, the diffuse radiation field and curvature terms of the velocity law. We will briefly comment on these restrictions and their consequences in Sect. 6. Since these approximations are generally used in the considered framework and may influence the results only under very specific conditions, we accept them until further discussion. Note already here, however, that by neglecting the influence of line-overlap we can and will describe only the situation for "normal" stars, whereas the case of WRs requires additional considerations (e.g., Lucy \& Abbott 1993; Schmutz 1997; Springmann \& Puls 1998 and Owocki \& Gayley 1999);

- The classification of lines to be either optically thin or thick with interaction probabilities of $\tau$ or unity, respectively, instead of using the "exact" expression $(1-\exp (-\tau)$, see below) leads to an only marginal error. We prefer this procedure throughout the major part of our discussion since it yields more insight into the relevant physical processes;

- Finally, we consider only stationary and onedimensional winds, since our reasoning is directed to understanding the reaction of an ensemble of lines projected from the behaviour of a single line, and the usual procedure to allow for this projection is similar in cases of time-dependent (e.g., Owocki \& Rybicki 1984) or multidimensional (e.g., Owocki et al. 1994) calculations.

\subsection{The radiative acceleration provided by one line}

Within these approximations and assumptions, the radiative acceleration provided by scattering of photons in a single line (transition frequency $\nu_{\mathrm{i}}$ ) to the material in a spherically expanding shell of size $\mathrm{d} r$, mass $\mathrm{d} m=4 \pi \rho r^{2} \mathrm{~d} r$ and velocity $v(r) \ldots v(r)+\mathrm{d} v$ is given by the average transferred momentum per unit time and $\mathrm{d} m$, i.e.,

$$
\begin{aligned}
g_{\mathrm{rad}}^{\mathrm{i}} & =\frac{\langle\Delta P\rangle}{\Delta t \mathrm{~d} m}= \\
& =\frac{1}{4 \pi \rho r^{2} \mathrm{~d} r} N_{\nu} \mathrm{d} \nu \frac{h \nu}{c}\left(1-\exp \left(-\tau_{\mathrm{S}}\right)\right)= \\
& =\frac{1}{4 \pi r^{2} c^{2}} L_{\nu} \nu_{\mathrm{i}} \frac{1}{\rho} \frac{\mathrm{d} v}{\mathrm{~d} r}\left(1-\exp \left(-\tau_{\mathrm{S}}\right)\right)
\end{aligned}
$$

where $N_{\nu} \mathrm{d} \nu$ is the number of photospherically emitted photons per unit time in the frequency range $\nu \ldots \nu+\mathrm{d} \nu$, $L_{\nu}$ the stellar luminosity and

$$
\begin{aligned}
\langle\Delta P\rangle & =N_{\nu} \mathrm{d} \nu \frac{h \nu}{c}= \\
& =\frac{L_{\nu} \mathrm{d} \nu}{h \nu} \frac{h \nu}{c}
\end{aligned}
$$

the average transferred momentum by line absorption or scattering (radially streaming photons provided and accounting for the cancellation of the foreaft-symmetric reemission processes). Finally,

$\mathrm{d} \nu=\nu_{\mathrm{i}} \frac{\mathrm{d} v}{c}$

is the frequency range which can actually contribute to line scattering inside the shell via the Doppler effect, and $\left(1-\exp \left(-\tau_{\mathrm{S}}\right)\right)$ is the interaction probability for a line optical depth (in Sobolev approximation)

$$
\begin{aligned}
& \tau_{\mathrm{S}}=\frac{\bar{\chi}_{\mathrm{i}} \lambda_{\mathrm{i}}}{\mathrm{d} v / \mathrm{d} r} \\
& \bar{\chi}_{\mathrm{i}}=\frac{\pi e^{2}}{m_{\mathrm{e}} c} g f_{\mathrm{i}}\left(\frac{n_{\mathrm{l}}}{g_{\mathrm{l}}}-\frac{n_{\mathrm{u}}}{g_{\mathrm{u}}}\right)
\end{aligned}
$$

with $\bar{\chi}_{\mathrm{i}}$ the frequency integrated line opacity, $g f$-value $g f_{\mathrm{i}}$ and $n_{1}, n_{\mathrm{u}}, g_{\mathrm{l}}, g_{\mathrm{u}}$ the occupation numbers and statistical weights of the participating lower and upper level.

\subsection{Line-strength and optical depth}

To proceed further, we have to sum up the contribution of all individual lines to obtain the total radiative acceleration. To this end, we define the dimensionless line-strength of a single line as

$$
\begin{aligned}
& k_{\mathrm{L}}=\frac{\chi_{\nu}\left(\nu_{\mathrm{i}}\right) \sqrt{\pi}}{\sigma_{\mathrm{e}}}=\frac{\bar{\chi}_{\mathrm{i}}}{\Delta \nu_{\mathrm{D}} \sigma_{\mathrm{e}}}=\frac{\bar{\chi}_{\mathrm{i}} \lambda_{\mathrm{i}}}{\rho} \frac{1}{s_{\mathrm{E}} v_{\mathrm{th}}} \\
& \chi_{\nu}=\bar{\chi}_{\mathrm{i}} \Phi_{\nu} ; \Phi_{\nu}=\frac{1}{\sqrt{\pi} \Delta \nu_{\mathrm{D}}} \mathrm{e}^{-\left(\frac{\nu-\nu_{\mathrm{i}}}{\Delta \nu_{\mathrm{D}}}\right)^{2}} .
\end{aligned}
$$


$\sigma_{\mathrm{e}}=: s_{\mathrm{E}} \rho$ is the Thomson scattering opacity, $\Delta \nu_{\mathrm{D}}$ the Doppler width of the line and $v_{\text {th }}$ the ionic thermal velocity. From Eq. (6), the line-strength can be interpreted twofold. On the one side, it is, except from a factor $\sqrt{\pi}$, the maximum opacity of the considered line in units of the minimum continuum opacity present in the wind (=Thomson). Alternatively, it can be considered, except a factor of $2 N, N=2 \ldots 4$, as the ratio of frequency integrated line opacity $\bar{\chi}_{\mathrm{i}}$ to minimum frequency integrated continuum opacity $2 N \Delta \nu_{\mathrm{D}} \sigma_{\mathrm{e}}$.

One comment is necessary here: The incidence of $v_{\text {th }}$ at this stage of reasoning seems to be "natural" in terms of understanding the physical meaning of line-strength. Moreover, it is actually needed if we require the latter to be dimensionless, which is important for our future statistical analysis. As it will turn out, however, $v_{\text {th }}$ will reappear in various combinations with other quantities after summing up the line-strength contributions of different metals, i.e., $v_{\text {th }}$ would be no longer unique due to its dependence on atomic mass. Thus, from now on we will concentrate on the most important aspect of $v_{\text {th }}$ in this context, namely that it has a dimension (also noting that its value is smaller than the sound-speed), however use a value independent of atomic mass, in particular, the value for hydrogen.

The relation of the so defined line-strength to Sobolev optical depth is given by

$\tau_{\mathrm{S}}=\frac{\sigma_{\mathrm{e}} v_{\mathrm{th}}}{\mathrm{d} v / \mathrm{d} r} k_{\mathrm{L}}=t k_{\mathrm{L}}$

where $t$ is the optical depth parameter defined by CAK. The advantage of using $k_{\mathrm{L}}$ instead of opacities or optical depths is that $k_{\mathrm{L}}$ is a quantity which remains rather constant throughout the wind (at least in the typical case of frozen-in ionization), and whose distribution can be described in an almost depth independent statistical way. Before doing this, however, we will sum up the contributions of all individual lines, in the spirit outlined above, i.e., by dividing lines into two categories. Optically thick lines are those with $\tau_{\mathrm{S}} \geq 1$ and interaction probability "1", whereas optically thin lines shall have $\tau_{\mathrm{S}}<1$ and interaction probability $\tau_{\mathrm{S}}$. Defining

$k_{1}=k_{\mathrm{L}}\left(\tau_{\mathrm{S}}=1\right)=\frac{\mathrm{d} v / \mathrm{d} r}{\sigma_{\mathrm{e}} v_{\mathrm{th}}}=\frac{1}{s_{\mathrm{E}} v_{\mathrm{th}}} \frac{\mathrm{d} v / \mathrm{d} r}{\rho}=t^{-1}$

as the line-strength where the division $\tau_{\mathrm{S}}=1$ is reached, we find for the total line acceleration

$g_{\text {rad }}^{\text {tot }}=\frac{s_{\mathrm{E}} v_{\text {th }}}{4 \pi r^{2} c^{2}}\left\{k_{1} \sum_{k_{\mathrm{L}}^{\mathrm{i}} \geq k_{1}} L_{\nu_{\mathrm{i}}} \nu_{\mathrm{i}}+\sum_{k_{\mathrm{L}}^{\mathrm{i}}<k_{1}} k_{\mathrm{L}}^{\mathrm{i}} L_{\nu_{\mathrm{i}}} \nu_{\mathrm{i}}\right\}$.

The first term inside the bracket gives the contribution by optically thick lines and depends only on the hydrodynamical structure via $k_{1} \propto(\mathrm{d} v / \mathrm{d} r) / \rho$, whereas the second term gives the optically thin line contribution and is independent of the hydro-structure, however depends on the specific line-strengths, i.e., atomic properties and level population.

\subsection{Line-strength distribution function and total acceleration}

\subsubsection{Perfect power-law distribution}

Before going into further detail and in concert with most previous investigations related to this topic, we will assume that the number of lines in a frequency interval $\nu, \nu+\mathrm{d} \nu$ and line-strength $k_{\mathrm{L}}, k_{\mathrm{L}}+\mathrm{d} k_{\mathrm{L}}$ can be represented by a power-law

$\mathrm{d} N\left(\nu, k_{\mathrm{L}}\right)=-N_{\mathrm{o}} f_{\nu}(\nu) k_{\mathrm{L}}^{\alpha-2} \mathrm{~d} \nu \mathrm{d} k_{\mathrm{L}}$,

with $0<\alpha<1$, where the frequential distribution shall be independent from the line-strength distribution. The negative sign accounts for the fact that the number of lines increases for decreasing line-strength. So far, the normalization "constant" $N_{\mathrm{o}}$ is allowed to have some additional depth dependence. With (10) and substituting the sums in (9) by appropriate (double-) integrals with frequencies from $0 \ldots \infty$ and line-strengths from $k_{1} \ldots \infty$ and $0 \ldots k_{1}$, respectively, we find

$$
\begin{aligned}
g_{\mathrm{rad}}^{\mathrm{tot}} & =\frac{s_{\mathrm{E}} v_{\mathrm{th}}}{4 \pi r^{2} c^{2}}\left\{k_{1} \int_{0}^{\infty} \int_{k_{1}}^{\infty} L_{\nu} \nu\left|\mathrm{d} N\left(\nu, k_{\mathrm{L}}\right)\right|+\right. \\
& \left.+\int_{0}^{\infty} \int_{0}^{k_{1}} k_{\mathrm{L}} L_{\nu} \nu\left|\mathrm{d} N\left(\nu, k_{\mathrm{L}}\right)\right|\right\}= \\
& =\frac{s_{\mathrm{E}} v_{\mathrm{th}} N_{\mathrm{o}} \int_{0}^{\infty} L_{\nu} \nu f_{\nu}(\nu) \mathrm{d} \nu}{4 \pi r^{2} c^{2}}\left\{\frac{1}{1-\alpha} k_{1}^{\alpha}+\frac{1}{\alpha} k_{1}^{\alpha}\right\}
\end{aligned}
$$

Note, that in case of $\alpha<0$ the contribution from optically thin lines (second term) would diverge at its lower boundary. From Eq. (12), two points are obvious: Both the line-force provided by optically thick and by optically thin lines scales with the same power $\alpha$, and we can interpret this exponent as the ratio of line-force from optically thick lines to total force,

$\alpha=\frac{g_{\mathrm{rad}}^{\mathrm{thick}}}{g_{\mathrm{rad}}^{\text {tot }}}$,

a result, which we will later on discuss carefully. Collecting terms and using the radiative acceleration provided by Thomson-scattering as a scaling factor,

$g_{\mathrm{rad}}^{\mathrm{TH}}=\frac{s_{\mathrm{E}} L}{4 \pi r^{2} c}$

with stellar luminosity $L$, we can express the total line acceleration in terms of the so-called force-multiplier f.m. $(r)$,

f.m. $(r)=\frac{g_{\mathrm{rad}}^{\mathrm{tot}}}{g_{\mathrm{rad}}^{\mathrm{TH}}}=k_{\mathrm{CAK}} k_{1}^{\alpha}=k_{\mathrm{CAK}} t^{-\alpha}$,

where the force-multiplier parameter $k_{\mathrm{CAK}}$ is defined by

$k_{\mathrm{CAK}}=\frac{\int_{0}^{\infty} L_{\nu} \nu f_{\nu}(\nu) \mathrm{d} \nu}{L} \frac{v_{\mathrm{th}}}{c} \frac{N_{\mathrm{o}}}{\alpha(1-\alpha)}$.

This result was given firstly by CAK. Note, however, that they additionally assumed $f_{\nu}(\nu) \mathrm{d} \nu=\mathrm{d} \nu / \nu$, in which case the first factor in (16) is unity. 
"Exact" result. Let us now consider the error we have made by dividing the lines into optically thick and thin ones exclusively. Accounting for the definition of the linestrength and its relation to the Sobolev optical depth, we can reformulate the radiative acceleration provided by one line, Eq. (1),

$$
\begin{aligned}
g_{\text {rad }}^{\mathrm{i}} & =\frac{s_{\mathrm{E}} v_{\mathrm{th}} L_{\nu} \nu_{\mathrm{i}}}{4 \pi r^{2} c^{2}} k_{\mathrm{L}}^{\mathrm{i}} \frac{1-\exp \left(-\tau_{\mathrm{S}}\right)}{\tau_{\mathrm{S}}}= \\
& =\frac{s_{\mathrm{E}} v_{\mathrm{th}} L_{\nu} \nu_{\mathrm{i}}}{4 \pi r^{2} c^{2}} k_{1}\left(1-\exp \left(-\frac{k_{\mathrm{L}}^{\mathrm{i}}}{k_{1}}\right)\right) .
\end{aligned}
$$

The first equation above can be interpreted as the product of the radiative acceleration if the line were optically thin, multiplied by the correction for self-shadowing due to the actual optical depth $\tau_{\mathrm{S}}$ (cf. also Gayley 1995). Integrating over the line-strength distribution function without performing the division at $\tau_{\mathrm{S}}=1$, we obtain directly the "exact" version of Eq. (12),

$g_{\mathrm{rad}}^{\mathrm{tot}}=\frac{s_{\mathrm{E}} v_{\mathrm{th}} N_{\mathrm{o}} \int_{0}^{\infty} L_{\nu} \nu f_{\nu}(\nu) \mathrm{d} \nu}{4 \pi r^{2} c^{2}} \frac{\Gamma(\alpha)}{1-\alpha} k_{1}^{\alpha}$

with Gamma-function $\Gamma(\alpha)$. Thus, since $0<\alpha<1$ (by assumption), the error introduced by our approximation is given by $1 /(\alpha \Gamma(\alpha))=1 /(\Gamma(1+\alpha))$, i.e., an overestimation of at most $13 \%$.

\subsubsection{Distribution functions with arbitrary dependence} on line-strength

In the previous section, we have shown that the assumption of power-law distributed line-strengths with exponent $\alpha-2$ directly leads to an ensemble line-force being proportional to $k_{1}^{\alpha}$, and that in this case $\alpha$ turns out to represent the ratio of optically thick to total line acceleration, provided that $0<\alpha<1$.

In so far, it seems quite natural to consider line strength distribution function and line force as interchangeable quantities and to identify the line-force exponent instantaneously as a manifestation of the underlying line-statistics. Although this perspective is widely spread, a closer inspection of the above procedure immediately necessitates a major caveat:

Due to the different weighting of line-strength in the expression for the line-force (Eq. 11), $g_{\mathrm{rad}}^{\text {thin }} \propto \int k_{\mathrm{L}} \mathrm{d} N\left(k_{\mathrm{L}}\right)$ and $g_{\text {rad }}^{\text {thick }} \propto \int \mathrm{d} N\left(k_{\mathrm{L}}\right)$, a strict correspondence between the exponent of line-strength distribution and forcemultiplier parameter can be expected a priori only if the distribution follows a power-law over a large range of line-strengths. If the distribution is curved in the log, this equality remains questionable and has to be considered with caution, even if one accounts for locally defined values $\alpha=\alpha\left(k_{\mathrm{L}}=k_{1}\right)$.

To investigate this problem, we consider a generalized line-strength distribution function

$\mathrm{d} N\left(\nu, k_{\mathrm{L}}\right)=-f\left(k_{\mathrm{L}}, \nu\right) \mathrm{d} \nu \mathrm{d} k_{\mathrm{L}}$, with arbitrary (positive) function $f\left(k_{\mathrm{L}}, \nu\right)$, where we even allow for a dependence of line-strength on frequency. Then, by realizing that

$\left\langle N\left(k_{\mathrm{L}}\right)\right\rangle=\int_{k_{\mathrm{L}}}^{\infty} \mathrm{d} k_{\mathrm{L}}^{\prime}\left(\int_{0}^{\infty} \frac{L_{\nu} \nu}{L} f\left(k_{\mathrm{L}}^{\prime}, \nu\right) \mathrm{d} \nu\right)$

is the flux (times frequency) weighted cumulative number of lines stronger than $k_{\mathrm{L}}$, we can integrate the optically thin contribution in (11) by parts and obtain, after adding the optically thick contribution

$g_{\text {rad }}^{\text {tot }} \propto \int_{0}^{k_{1}}\left\langle N\left(k_{\mathrm{L}}\right)\right\rangle \mathrm{d} k_{\mathrm{L}}$.

Here we have assumed that the total (flux weighted) number of lines $\langle N(0)\rangle$ remains finite. (Actually, the much weaker requirement that $k_{\mathrm{L}}\left\langle N\left(k_{\mathrm{L}}\right)\right\rangle \rightarrow 0$ for $k_{\mathrm{L}} \rightarrow 0$ is sufficient.)

Equation (21) can be alternatively expressed as

$g_{\text {rad }}^{\text {tot }}=\frac{L s_{\mathrm{E}} v_{\text {th }}}{4 \pi r^{2} c^{2}}\left\{k_{1}\left\langle N_{\text {thick }}\right\rangle+\int_{0}^{k_{1}}\left\langle N_{\text {thin }}\left(k_{\mathrm{L}}\right)\right\rangle \mathrm{d} k_{\mathrm{L}}\right\}$,

with $\left\langle N_{\text {thick }}\right\rangle=\left\langle N\left(k_{1}\right)\right\rangle$ the weighted number of lines stronger than $k_{1}$ (which are then optically thick by definition) and $\left\langle N_{\text {thin }}\left(k_{\mathrm{L}}\right)\right\rangle$ the cumulative number of optically thin lines $\left(=\left\langle N\left(k_{\mathrm{L}}\right)\right\rangle-\left\langle N\left(k_{1}\right)\right\rangle\right)$. Figure 1 displays this result graphically. Note, that in order to derive this result, we have again used our approximation of replacing the interaction probability $1-\exp \left(-\tau_{\mathrm{S}}\right)$ by $\left(1, \tau_{\mathrm{S}}\right)$, respectively.

Before we proceed further, let us mention that Eq. (22) and Fig. 1 allow for an useful visualization of two extreme cases. At first, assume that either the velocity gradient is so small or the density so high that all lines are effectively stronger than/equal to $k_{1}$. In this optically thick case, $k_{1}$ is situated in the left part of the previous figure, and $\left\langle N\left(k_{\mathrm{L}}\right)\right\rangle=\left\langle N\left(k_{1}\right)\right\rangle=$ const for all $k_{\mathrm{L}}<k_{1}$ by definition (no lines weaker than $k_{1}$ present). The acceleration is then found from the rectangular area between $k_{\mathrm{L}}=0 \ldots k_{1}$, i.e., $g_{\mathrm{rad}}^{\text {tot }} \propto k_{1}$ and thus $\alpha=1$. From the figure, it is also clear that the resulting acceleration is enormously reduced compared to the case of all lines being optically thin (cf. also Eq. (17)), which is the other extreme. In the latter case (arising for large velocity gradients or low densities), $k_{1}$ lies at the rightmost point of the abscissa, and all lines have strengths lower than $k_{1}$. Hence, the integral becomes independent of $k_{1}$, and the radiative acceleration obtains its maximum value with $\alpha=0$. In all other cases, the $\alpha$-parameter of the line-force corresponds to the ratio of "dark" area (optically thick force) and the sum of light and dark area (total force), cf. Eq. (13) for the case of a strict power-law and Sect. 2.4 otherwise.

On the basis of our alternative and general expression (21) for the line-force, we can now answer the question raised above, namely under which conditions this line-force can be represented by the CAK law $g_{\text {rad }}^{\text {tot }} \propto k_{1}^{\alpha}$, where $\alpha$ corresponds at least to some local exponent of our arbitrary line-strength distribution function. 


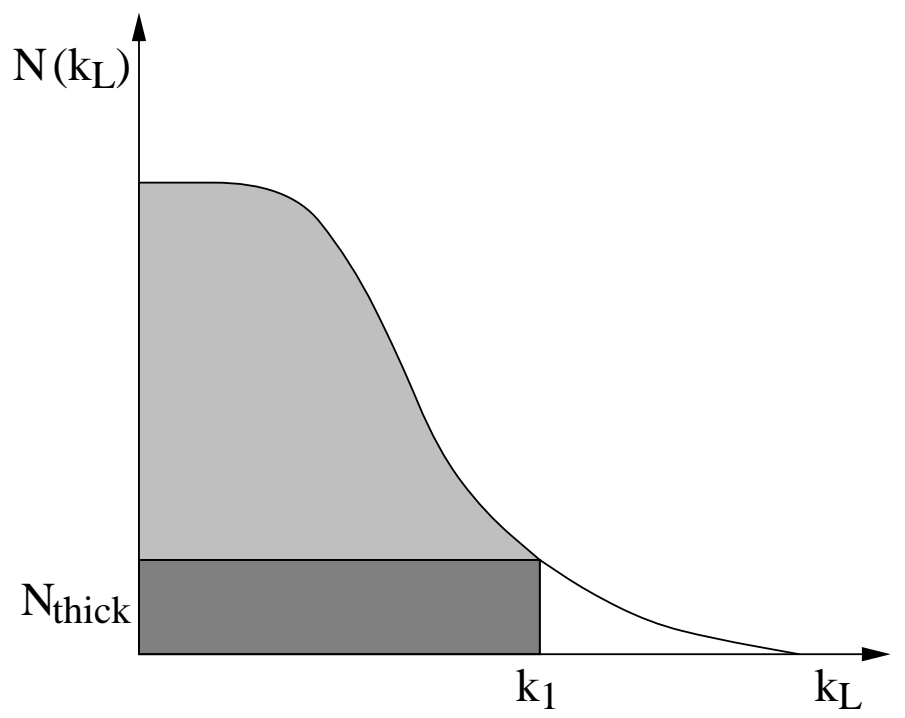

Fig. 1. The line-force as integral of (flux weighted) cumulative line number over line-strength; contribution by optically thin (light) and optically thick lines (dark). Compare Eq. (22) and text

To this end, we define two line-strengths $k_{-}<k_{1}<$ $k_{+}$, where $k_{-}$and $k_{+}$are chosen in such a way that $N\left(k_{\mathrm{L}}\right)$ follows roughly a power-law in between, i.e., is roughly linear with slope $\alpha-1$ in the log-log representation. Actually, this is almost always possible if the range $k_{-} \ldots k_{+}$is not too large, say of order two dex. (Here and in the following we assume that the wind is not too thin, so that $k_{1}$ lies well below the maximum line-strength.)

The number of lines at $k_{+}$is then $N_{+}, N_{-}$is $N\left(k_{-}\right)$ (flux weighting always provided, however brackets suppressed to simplify notation) and the distribution function in between can be approximated by

$$
\begin{aligned}
N\left(k_{\mathrm{L}}\right) & =N_{+} k_{+}^{1-\alpha} k_{\mathrm{L}}^{\alpha-1} ; k_{-}<k_{\mathrm{L}}<k_{+} \\
\alpha & =1+\frac{\log \left(N_{+} / N_{-}\right)}{\log \left(k_{+} / k_{-}\right)} .
\end{aligned}
$$

Since by definition $N_{-} \geq N_{+}$and $k_{+}>k_{-}$, the maximum value of $\alpha$ is constrained to be unity, which occurs in those cases when the maximum line number is reached at a certain $k_{\mathrm{L}}$-value. $\alpha$-values below zero are not excluded from now on in our local description.

Under these conditions and using (22), the total line acceleration is given by

$$
\begin{aligned}
& g_{\text {rad }}^{\text {tot }} \propto \int_{0}^{k_{-}} N\left(k_{\mathrm{L}}\right) \mathrm{d} k_{\mathrm{L}}+\int_{k_{-}}^{k_{1}} N_{+} k_{+}^{1-\alpha} k_{\mathrm{L}}^{\alpha-1} \mathrm{~d} k_{\mathrm{L}} \\
& =\left\{\bar{N}_{\left\{0, k_{-}\right\}}-\frac{N_{+}}{\alpha}\left(\frac{k_{+}}{k_{-}}\right)^{1-\alpha}\right\} k_{-}+\left(\frac{N_{+} k_{+}^{1-\alpha}}{\alpha}\right) k_{1}^{\alpha}
\end{aligned}
$$

( $\alpha \neq 0$ ), where $\bar{N}_{\left\{0, k_{-}\right\}}$is the appropriate average of $N\left(k_{\mathrm{L}}\right)$ in the range $0 \ldots k_{-}$

$\bar{N}_{\left\{0, k_{-}\right\}} k_{-}=: \int_{0}^{k_{-}} N\left(k_{\mathrm{L}}\right) \mathrm{d} k_{\mathrm{L}}$.
From Eq. (24), it is obvious that the ensemble line-force can be represented by $k_{1}^{\alpha}$ with local $\alpha$, if and only if the first term is small compared to the second one, where the former is just the difference between actual and "fitted" area (i.e., acceleration) in the range $0 \ldots k_{-}$.

In Appendix A, we show that under fairly general assumptions this is actually the case if $\alpha>0$, i.e., as long as the local slope of the flux-weighted cumulative line-strength distribution (in the log-log representation) is larger than -1 . In contrast, line distributions with a steep slope over a large $k_{\mathrm{L}}$ range will decouple from the line-force parameterization (cf. also Sect. 4.2.8), leading to effective $\alpha$ values in the line-force (see below) different from those defining the line-strength statistics.

\subsection{Effective value of $\alpha$}

Usually, the force multiplier parameters are not derived from the line-strength distribution function, however from the line-force itself, i.e., accouting explicitely for the additional weighting with $k_{\mathrm{L}}$ mentioned above.

Thus, in the following we postulate the line force to be a function of $k_{1}^{\alpha}$ with no a priori knowledge of $\alpha$. Instead, we define an effective value $\hat{\alpha}^{1}$ by

$\hat{\alpha}=\frac{\mathrm{d} \ln g_{\mathrm{rad}}^{\mathrm{tot}}}{\mathrm{d} \ln k_{1}}=\frac{\mathrm{d} g_{\mathrm{rad}}^{\mathrm{tot}}}{\mathrm{d} k_{1}} \frac{k_{1}}{g_{\mathrm{rad}}^{\mathrm{tot}}}$

From this definition and using ensemble line-forces calculated by summing up their individual components (e.g., Abbott 1982; Pauldrach et al. 1994), we obtain by straightforward differentiation (cf. Eq. 17)

$\frac{\mathrm{d} g_{\mathrm{rad}}^{\mathrm{tot}}}{\mathrm{d} k_{1}}=\frac{s_{\mathrm{E}} v_{\mathrm{th}}}{4 \pi r^{2} c^{2}} \frac{\mathrm{d}}{\mathrm{d} k_{1}} \sum_{i} k_{1}\left(1-\exp \left(-\frac{k_{\mathrm{L}}^{\mathrm{i}}}{k_{1}}\right)\right) L_{\nu_{\mathrm{i}}} \nu_{\mathrm{i}}=$

$=\frac{s_{\mathrm{E}} v_{\mathrm{th}}}{4 \pi r^{2} c^{2}} \sum_{i_{\mathrm{t}}}\left(1-\exp \left(-\frac{k_{\mathrm{L}}^{\mathrm{i}}}{k_{1}}\right)-\frac{k_{\mathrm{L}}^{\mathrm{i}}}{k_{1}} \exp \left(-\frac{k_{\mathrm{L}}^{\mathrm{i}}}{k_{1}}\right)\right) L_{\nu_{\mathrm{i}}} \nu_{\mathrm{i}}$

$\approx \frac{g_{\mathrm{rad}}^{\mathrm{tot}}}{k_{1}}-\frac{g_{\mathrm{rad}}^{\mathrm{thin}}}{k_{1}}=\frac{g_{\mathrm{rad}}^{\mathrm{thick}}}{k_{1}}$

where we have performed our approximation $1-\exp \left(-\tau_{\mathrm{S}}\right)=\left(1, \tau_{\mathrm{S}}\right)$ for $k_{\mathrm{L}}^{\mathrm{i}}>k_{1}, k_{\mathrm{L}}^{\mathrm{i}}<k_{1}$, respectively, and neglected 2nd order terms. Hence, from Eq. (26)

$\hat{\alpha} \approx \frac{g_{\mathrm{rad}}^{\mathrm{thick}}}{g_{\mathrm{rad}}^{\mathrm{tot}}}$

we find the same result as in Eq. (13), however independent of any underlying line statistics! Especially, this result does not rely on any separability of frequency and line-strength ${ }^{2}$.

\footnotetext{
${ }^{1}$ From here on, all line-force parameters derived from a given or calculated force itself will be denoted with an additional circumflex, in contrast to those parameters related or resulting from a specific line statistics.

${ }^{2}$ The same result can be found by applying definition (26) to our alternative expression for the
} 
In a first interpretation of $\hat{\alpha}$, Abbott (1980, his Eq. 10) found a result different from our Eq. (28). This difference, however, bases on Abbott's implicit assumption that the average (flux times frequency weighted) line-strength of optically thin lines,

$\bar{k}_{\text {thin }}=\frac{\sum_{k_{\mathrm{L}}^{\mathrm{i}}<k_{1}} k_{\mathrm{L}}^{\mathrm{i}} L_{\nu_{\mathrm{i}}} \nu_{\mathrm{i}}}{\sum_{k_{\mathrm{L}}^{\mathrm{i}}<k_{1}} L_{\nu_{\mathrm{i}}} \nu_{\mathrm{i}}}$

does not depend on the hydro variable $k_{1}$. If one relaxes this assumption and accounts also for the variation of $\bar{k}_{\text {thin }}$ as function of $k_{1}$, the nominator in Abbott's Eq. (10) becomes unity and the same result for $\hat{\alpha}$ is recovered as given in (28).

From this expression, it is obvious that $\hat{\alpha}$ must lie in the range $0 \ldots 1$. Combined with our previous notion that the line-force can be parameterized in the form $k_{1}^{\alpha}$ if the local $\alpha$ value of the distribution function is larger than zero, this leads to the result that we should have $\hat{\alpha}\left(k_{1}\right) \approx \alpha\left(k_{1}\right)$ for a large range of line-strengths as long as this condition is met. If the distribution has a significant steepness locally, $\hat{\alpha}\left(k_{1}\right)>\alpha\left(k_{1}\right)$ is to be expected.

Furthermore, the value of $\hat{\alpha}$ is independent of $k_{1}$ if and only if the flux-weighted line-strength distribution follows an exact power law with exponent $\alpha-2$. In this case then, $\hat{\alpha}=\alpha$ globally. Otherwise, $\hat{\alpha}$ becomes a function of $k_{1}$ and thus a function of depth. An instructive example is given in Appendix B.

\subsection{The interpretation of $\hat{\alpha}$ : Acceleration vs. line-number ratio}

It has often been argued that $\hat{\alpha}$ is closely related to the number ratio of optically thick to thin lines, contrasted to the above formulated acceleration ratio. In the following, however, we will show that the former gives little (if any) insight into the behaviour of $\hat{\alpha}$.

At first note that in view of Eq. (11) the optically thin line-force can be expressed by

$g_{\text {rad }}^{\text {thin }} \propto\left\langle N_{\text {thin }}\right\rangle \bar{k}_{\text {thin }}$.

Thus, the product of (flux weighted) number of optically thin lines times average line-strength $\left\langle N_{\text {thin }}\right\rangle \bar{k}_{\text {thin }}$ remains bounded, although the number itself may formally diverge, e.g. for a typical power-law index $\alpha-2<-1$. In this case then, the average line-strength of optically thin lines $\bar{k}_{\text {thin }}$ approaches zero!

To avoid this possible divergence and also to keep the computational effort as small as possible, one usually defines a minimum line-strength $k_{\min }<k_{1}$ as a lower boundary for the contributing lines, resulting in a modified acceleration

$g_{\mathrm{rad}}^{\mathrm{thin}}\left(k_{\min }\right) \propto \frac{k_{1}^{\alpha}}{\alpha}\left(1-\left(\frac{k_{\min }}{k_{1}}\right)^{\alpha}\right)$.

line acceleration (21), again independent from any assumption for the line statistics. Note also, that Gayley (1995) defined the effective $\alpha$ value at the critical point by the same expression.
If one allows for a relative error $\epsilon$ in the line-force and supposes the power-law distribution function to be valid throughout the range $0<k<\infty$, one finds as a constraint for $k_{\min }$

$\frac{k_{\min }}{k_{1}}=\left(\frac{\epsilon}{1-\alpha}\right)^{\frac{1}{\alpha}}$.

Hence and under "normal" conditions $\left(k_{1}=\mathrm{O}(1000)\right)$, it is sufficient to account for lines stronger than $k_{\min }=1$, i.e., stronger than Thomson-scattering, if one calculates the line-force (this was done, e.g., in Fig. A1). However, in cases of high wind densities, this limit has to be lowered, since the contribution of weaker lines with $k_{\mathrm{L}}<1$ becomes considerable then.

Using this cutoff, the number ratio of optically thick to thin lines is given by

$\frac{N_{\text {thick }}}{N_{\text {thin }}}=\frac{1}{\left(k_{1} / k_{\min }\right)^{1-\alpha}-1} \rightarrow 0$ for $k_{\min } \rightarrow 0$.

Thus, it depends strongly on $k_{1}$ (usually increasing throughout the wind) as well as on the value chosen for $k_{\min }$, contrasted to the accordingly modified acceleration ratio

$\frac{g_{\mathrm{rad}}^{\text {thick }}}{g_{\mathrm{rad}}^{\text {thin }}}=\frac{\alpha}{1-\alpha} \frac{1}{1-\left(k_{\min } / k_{1}\right)^{\alpha}} \rightarrow \frac{\alpha}{1-\alpha}$ for $k_{\min } \rightarrow 0$

The latter limit, of course, is only valid for $\alpha>0$. In consequence, even for the simple picture of a perfect power-law, the "knowledge" or anticipated behaviour of $N_{\text {thick }} / N_{\text {thin }}$ gives only little (if any) insight into the value of $\hat{\alpha}$. E.g., the expectation that $N_{\text {thick }} / N_{\text {thin }} \ll 1$ implies $\hat{\alpha} \rightarrow 0$ is, in view of Eq. (33), by no means justified. As one example of this kind of misinterpretation, we want to mention the argument given by Kudritzki et al. (1987) to explain the lower $\hat{\alpha}$-values resulting from NLTE-calculations for winds with reduced metallicity. It was argued that this effect can be "easily understood in terms of the metallicity", since the "ratio of strong to weak lines must decrease accordingly" if the metallicity is lowered. With respect to Eq. (33), this argument is simply wrong, since the plain number ratio becomes inevitably smaller for a reduced wind density ( $k_{1}$ larger) and thus cannot be used to give any predictions concerning $\hat{\alpha}$. What really matters - if one prefers a discussion in terms of line numbers - is the ratio of optically thick lines to the average number of lines in the range $k_{\mathrm{L}}=0 \ldots k_{1}$,

$\hat{\alpha}=\frac{\left\langle N_{\text {thick }}\right\rangle}{\left\langle\bar{N}_{\left\{0, k_{1}\right\}}\right\rangle}$,

by means of Eq. (21). Further comments on the behaviour of $\hat{\alpha}$ in a low metallicity environment are given in Sect. 5 .

\subsection{Relation to Gayley's $\bar{Q}$}

As we have mentioned in the introduction, Gayley (1995) considered the problem of radiative line driving in a concept somewhat different from the conventional approach. 
After discussing the physical origin why radiative line driving is so much more efficient than the radiative continuum acceleration, he introduces the meanwhile well known quantity $\bar{Q}$ and relates it to the alternative modified CAK approach. The reason that his approach seems to be somewhat favourable compared to the latter is his finding that $\bar{Q}$ should be much more constant than the line-force parameter $k_{\mathrm{CAK}}$, which is somewhat messed up with implicit dependencies on $\alpha$, cf. Eq. (16). By comparing with various published values of force-multipliers, he concludes that $\bar{Q}$ should be of the order 1000 to 2000 . In order to compare to his approach and since in the remaining part of the paper we are mostly concerned with the parameter $\alpha$, we will briefly relate his findings to our concept in the following, and comment on some problems if one considers realistic line-distribution functions in Sect. 3.3.

At first note that Gayley's line-strength parameter $q$ relates to our definition as

$q=\frac{v_{\mathrm{th}}}{c} k_{\mathrm{L}}$

where we have argued previously that in our notation $v_{\text {th }}$ is evaluated for hydrogen, i.e., is temperature, however not mass dependent.

Similarly, Gayley's cutoff parameter $Q_{\mathrm{o}}$ deviates from our quantity $k_{\max }$ (Eq. B1) by the same factor. Noting these correspondences and keeping our radial streaming approximation, $\bar{Q}$ is defined by

$$
\begin{aligned}
\bar{Q} & =\sum_{\text {all lines }} \frac{L_{\nu_{\mathrm{i}}} \nu_{\mathrm{i}}}{L} q_{i}=\frac{v_{\text {th }}}{c} \sum \frac{L_{\nu_{\mathrm{i}}} \nu_{\mathrm{i}}}{L} k_{\mathrm{L}}^{\mathrm{i}} \\
& \rightarrow \frac{v_{\text {th }}}{c} \int_{0}^{\infty} \int_{0}^{\infty} k_{\mathrm{L}} \frac{L_{\nu} \nu}{L}\left|\mathrm{~d} N\left(k_{\mathrm{L}}, \nu\right)\right|
\end{aligned}
$$

which is nothing else than the force-multiplier if all lines were optically thin (cf. Eq. 11). On the other hand, from Eq. (16) we find an intuitive interpretation of $k_{\mathrm{CAK}}$, which is roughly the fraction of the total stellar flux which would be blocked already in the photosphere if all lines were optically thick (assuming that each optically thick line blocks a fraction $L_{\nu} \Delta \nu_{\mathrm{D}}$ ), divided by $\alpha$. In so far, the physical upper limit of $k_{\mathrm{CAK}}$ is of order $1 / \alpha$. (Much earlier, however, the lines would overlap in the wind, and this effect would have to be accounted for, e.g. Puls 1987.)

With definition (37) and using a line-distribution function with exponential cutoff at $Q_{\mathrm{o}}$ (cf. B1) in order to prevent the number of strong lines from becoming smaller than unity, Gayley showed the correspondence (his Eq. (56))

$k_{\mathrm{CAK}}=\frac{1}{1-\alpha}\left(\frac{v_{\mathrm{th}}}{c}\right)^{\alpha} \bar{Q} Q_{\mathrm{o}}^{-\alpha}=\frac{\bar{Q}}{1-\alpha} k_{\max }^{-\alpha}$

in our notation. So far, the $\bar{Q}$ formalism seems to be of no major conceptual advantage compared to the CAK formalism. The interesting point, however, is the following: For power-law distributed line-strength distribution functions with (roughly) constant $\alpha$ and conditions valid for hot winds, one easily finds (cf. Sect. 3.3 and Appendix C) that

$\bar{Q} \approx Q_{\mathrm{o}}$

a relation which was invoked by Gayley as a generally valid Ansatz, i.e.,

$k_{\mathrm{CAK}}=\frac{1}{1-\alpha}\left(\frac{v_{\mathrm{th}}}{c}\right)^{\alpha} \bar{Q}^{1-\alpha}$.

If this Ansatz were correct and since $\bar{Q}$ is rather constant (cf. also Sect. 3.2), all scaling laws for mass-loss rates would become much easier to interpret and would especially depend only on the quantity $\alpha$ via

$\frac{\dot{M}}{L} \propto \frac{\alpha}{1-\alpha} F_{\mathrm{c}}^{\frac{1}{\alpha}}\left(\frac{\Gamma \bar{Q}}{1-\Gamma}\right)^{\frac{1}{\alpha}-1}$

(Gayley's Eq. (43), with stellar continuum flux $F_{\mathrm{c}}$ and usual Eddington $\Gamma$ ). In contrast, the standard formulation (in the same normalization) implies

$\frac{\dot{M}}{L} \propto \frac{\alpha v_{\mathrm{th}}}{c}\left(k_{\mathrm{CAK}} F_{\mathrm{c}}\right)^{\frac{1}{\alpha}}\left(\frac{(1-\alpha) \Gamma}{1-\Gamma}\right)^{\frac{1}{\alpha}-1}$

with a much more varying value of $k_{\mathrm{CAK}}$. (Actually, both expressions have to be slightly modified for the so-called $\delta$-term accounting for ionization effects, if present, cf. Abbott 1982 and Kudritzki et al. 1989). By comparing both equations, the different philosophy of $\bar{Q}$ and $k_{\mathrm{CAK}}$ is evident: the force-multiplier like quantity $\bar{Q}$ acts on the acceleration ratio $\Gamma$, whereas the flux-ratio like quantity $k_{\mathrm{CAK}}$ acts on the stellar flux!

If, on the other hand, $\bar{Q}$ differs significantly from $Q_{\mathrm{o}}$, Eq. (41) has to be modified by an additional factor $\bar{Q} / Q_{\mathrm{o}}$, and the gain of the $\bar{Q}$ formalism were lost, since then the variation in $k_{\mathrm{CAK}}$ would be found again in a variation of $Q_{\mathrm{o}}$. In how far this might be a problem will be discussed in the next section, after we have described how we calculate line-distribution functions and derive the appropriate force-multiplier parameters.

\section{Line-strength distribution functions for stellar wind conditions and the validity of Gayley's Ansatz}

In the previous sections, we have shown that under quite general conditions the summation of individual line accelerations leads to the CAK law $g_{\text {rad }}^{\text {tot }} \propto k_{1}^{\hat{\alpha}}$, where $\hat{\alpha}$ follows the local slope of the line-strength distribution function as long as this is not too steep.

Although the limiting values of $\hat{\alpha}=0 \ldots 1$ are obvious by definition, nothing has been said so far concerning its specific value. The importance of this quantity has been pointed out in the introduction and shall be stressed once more: To understand the basic wind physics and to be able to obtain quantitative predictions (as, e.g., via Eqs. (41) or (42)), a thorough discussion of the line-strength distribution and its dependence on different quantities such as wind density, metallicity etc. is inevitable. Before doing 
this in Sects. 4 and 5, we will describe our method of calculating those distribution functions, derive force-multiplier parameters and comment on the presumed equality of $\bar{Q}$ and $Q_{\mathrm{o}}$.

\subsection{Data and basic approximations}

\subsubsection{Atomic data}

The data base upon which this work is based has been compiled over the last 15 years by A. Pauldrach in collaboration with one of us (M.L.). The wavelengths, $g f$-values, photoionization cross sections and collision strengths for a total of 149 ionization stages and 2.5 million lines are stored (for the highest ionization stage of the elements see Table 1). The considered elements are hydrogen to zinc except lithium, beryllium, boron and scandium which are too rare to play a role in radiative line driving. The origin of the data has recently been described by Pauldrach et al. (1998). Note, that each model ion considered in NLTE consists of carefully chosen levels (typically of order 50), which are sufficient to define the most important occupation numbers required for calculating the line-force, as long as the line list is complete. For light ions, the highest considered level lies close to the ionization edge, whereas for the heavy elements the cutoff was chosen in such a way to include all meta-stable levels and levels above which are significantly populated.

Of course, the completeness of the data in terms of their potential contribution to radiative driving is a critical issue. Apart from the high frequency cutoff given by the highest represented ionization stages (thereby effectively limiting the usefulness of the line database for computing radiation pressure to stars with $\left.T_{\text {eff }}<100000 \mathrm{~K}\right)$ there is the question of how many weak lines have to be represented to regard the list as essentially complete. Comparisons have been made (see Springmann 1997) with the line opacity data from the Opacity Project (Seaton 1995) and the Kurucz data (Kurucz 1995). After gaps in line opacity due to missing data in the UV spectral range in our database were closed, all three data collections now agree in their spectral line opacity distribution.

Since the Kurucz data base is the most complete now in existence we conclude that we are as complete as presently possible. Furthermore, tests made by omitting the weakest lines have shown that their contribution is negligible so that further enhancements of line opacity redward of $229 \AA$ are not expected.

\subsubsection{Approximate non-LTE occupation numbers}

To determine the line-strengths for atomic transitions under stellar wind conditions one has to know the occupation numbers of the corresponding levels (see Eq. 5). To keep
Table 1. The highest ionization stage considered in our database. Carbon to Calcium: "light ions"; Titanium to Zinc: "iron group elements"

\begin{tabular}{llllll}
\hline \hline Elem. & max. ion. & Elem. & max. ion. & Elem. & m. ion. \\
\hline $\mathrm{H}$ & $\mathrm{I}$ & $\mathrm{He}$ & $\mathrm{II}$ & & \\
& & & & & \\
$\mathrm{C}$ & $\mathrm{V}$ & $\mathrm{N}$ & $\mathrm{VI}$ & $\mathrm{O}$ & $\mathrm{VI}$ \\
$\mathrm{F}$ & $\mathrm{VI}$ & $\mathrm{Ne}$ & $\mathrm{VI}$ & $\mathrm{Na}$ & $\mathrm{VI}$ \\
$\mathrm{Mg}$ & $\mathrm{VI}$ & $\mathrm{Al}$ & $\mathrm{VI}$ & $\mathrm{Si}$ & $\mathrm{VI}$ \\
$\mathrm{P}$ & $\mathrm{VI}$ & $\mathrm{S}$ & $\mathrm{VII}$ & $\mathrm{Cl}$ & $\mathrm{VI}$ \\
$\mathrm{Ar}$ & $\mathrm{VIII}$ & $\mathrm{K}$ & $\mathrm{VI}$ & $\mathrm{Ca}$ & $\mathrm{VI}$ \\
& & & & & \\
$\mathrm{Ti}$ & $\mathrm{V}$ & $\mathrm{V}$ & $\mathrm{V}$ & $\mathrm{Cr}$ & $\mathrm{VI}$ \\
$\mathrm{Mn}$ & $\mathrm{VI}$ & $\mathrm{Fe}$ & $\mathrm{VIII}$ & $\mathrm{Co}$ & $\mathrm{VII}$ \\
$\mathrm{Ni}$ & $\mathrm{VIII}$ & $\mathrm{Cu}$ & $\mathrm{VI}$ & $\mathrm{Zn}$ & $\mathrm{III}$ \\
\hline
\end{tabular}

matters simple we have employed the following assumptions (for a thorough discussion, cf. Springmann 1997):

Ionization equilibrium. The ionizing radiation field is approximated by $J_{\nu}=W I_{\nu}\left(T_{\mathrm{rad}}\right)$, where the intensity $I_{\nu}$ is taken either as Planck or from a Kurucz model atmosphere (Kurucz 1995). Since the atmospheric conditions are specified at one point only, the dilution factor is a numerical factor of order $1 \ldots 0.001$. With the electron temperature taken as a constant fraction of the effective temperature (typically 0.8) and the radiation temperature as either the effective one (Planck case) or lower (Kurucz fluxes), the ionization equilibrium reads

$$
\begin{aligned}
\frac{n_{1, j+1} n_{\mathrm{e}}}{n_{1, j}}= & W \sqrt{\frac{T_{\mathrm{e}}}{T_{\mathrm{rad}}}}\left(\frac{n_{1, j+1} n_{\mathrm{e}}}{n_{1, j}}\right)_{T_{\mathrm{rad}}}^{*} \\
& \times\{\zeta+\eta+W(1-\eta-\zeta)\}
\end{aligned}
$$

The asterisk denotes thermodynamic equilibrium values and $\zeta$ and $\eta$ are the fraction of recombination processes leading directly to the ground and meta-stable levels, respectively. The underlying assumptions leading to this equation (which goes back to unpublished notes by Leon Lucy) are: The ionization balance is dominated by radiative processes and given by the equilibrium of photoionization processes from all levels and recombination processes to all levels. Line transitions are considered as optically thin (i.e., the action of line transitions on the level populations is neglected; see below). The frequency dependence of the photoionization cross section is taken as a quadratic decline from the edge value; tests have shown that for a wide variety of parameterizations the error incurred does not exceed $10 \%$. Equation (43) has a smooth transition to the LTE-Regime for $W \rightarrow 1$. A similar equation (but without the $\eta$-terms) has been employed by Schmutz (1991) and Schaerer \& Schmutz (1994). 
Level occupation numbers. Having determined the ionization equilibrium, the distribution of the ions on the level states follows the Abbott \& Lucy (1985) prescription: meta-stable states have equilibrium populations relative to the ground state $\left(n_{m} / n_{1}=\left(n_{m} / n_{1}\right)_{T_{\text {rad }}}^{*}\right)$, other levels have a diluted population $\left(n_{i} / n_{l}=W\left(n_{i} / n_{l}\right)_{T_{\text {rad }}}^{*}\right)$ relative to either the ground state or a meta-stable state, depending on which lower state corresponds to the strongest downward transition. Excited levels which do not have a direct downward transition to either the ground or a metastable level are neglected. The transitions which have one of the three classes of levels as lower levels (i.e., resonance transitions, quasi-resonance transitions starting from a meta-stable level and 1st order subordinate transitions) contribute most of the line opacity. In this way it is possible to specify the level occupations without actually solving the rate equations.

This prescription for the the level occupations can be justified by considering a 3-level atom neglecting collisions and line optical thickness (in large distances from the star the mean intensity in optically thick lines decreases faster than in optically thin lines). This last assumption is hardly important, however, since it mainly affects the upper levels of a transition which have a negligible influence on the line optical thickness. Meta-stable levels are not affected since they are populated from higher levels (direct downward transitions are forbidden by definition). Collisions are important for high densities but here our prescription ensures a smooth transition to LTE both for the ionization and excitation structure. These assumptions are of greater importance when computing complete wind models with a radial stratification in all variables whereas for our present purposes they do not matter since we do not consider a specific model.

The end result of all approximations compares favourably with the much more detailed non-LTEcalculations by Pauldrach et al. (1994) with respect to both the ionization balance and the emergent flux (see the example for the O4If star $\zeta$-Pup in Springmann \& Puls 1998).

\subsection{Line-strength distribution functions and force-multiplier parameters for some examples}

Having calculated the occupation numbers for all involved levels, the line-strengths of all transitions in our data base can be found by means of Eq. (6) and the distribution functions derived. In the following sections, we will display either the differential form, where we bin (if not stated explicitly else) the number of lines $\Delta N$ per 0.5 dex in line-strength and 5 kiloKayser $\left(\mathrm{kK}, 1\right.$ Kayser $\left.=1 \mathrm{~cm}^{-1}\right)$ in frequency, or we show the cumulative line-strength distribution, i.e, the number of lines $N\left(k_{\mathrm{L}}\right)$ with strengths larger than $k_{\mathrm{L}}$. Flux (times frequency) weighted functions differ by the additional weight $\nu_{\mathrm{i}} F_{\nu} / F$, where $F$ is the integrated flux, assumed to be Planck in this section (using
Table 2. Various force-multiplier parameters as function of $T_{\text {eff }}$, for $n_{\mathrm{e} 11} / W=10$ and $W=0.33$ (see text)

\begin{tabular}{cccrrc}
\hline \hline$T_{\text {eff }}$ & $\log k_{\mathrm{CAK}}$ & $\hat{\alpha}$ & $Q$ & $Q_{\mathrm{o}}$ & $\begin{array}{l}\log k_{\mathrm{CAK}} \\
\left(Q_{\mathrm{o}}=\bar{Q}\right)\end{array}$ \\
\hline 50000 & -1.11 & 0.66 & 1939 & 2260 & -1.06 \\
40000 & -1.13 & 0.67 & 1954 & 1778 & -1.15 \\
30000 & -1.08 & 0.64 & 2498 & 3630 & -0.97 \\
20000 & -1.02 & 0.58 & 1597 & 5171 & -0.72 \\
10000 & -0.54 & 0.44 & 915 & 14505 & -0.01 \\
\hline
\end{tabular}

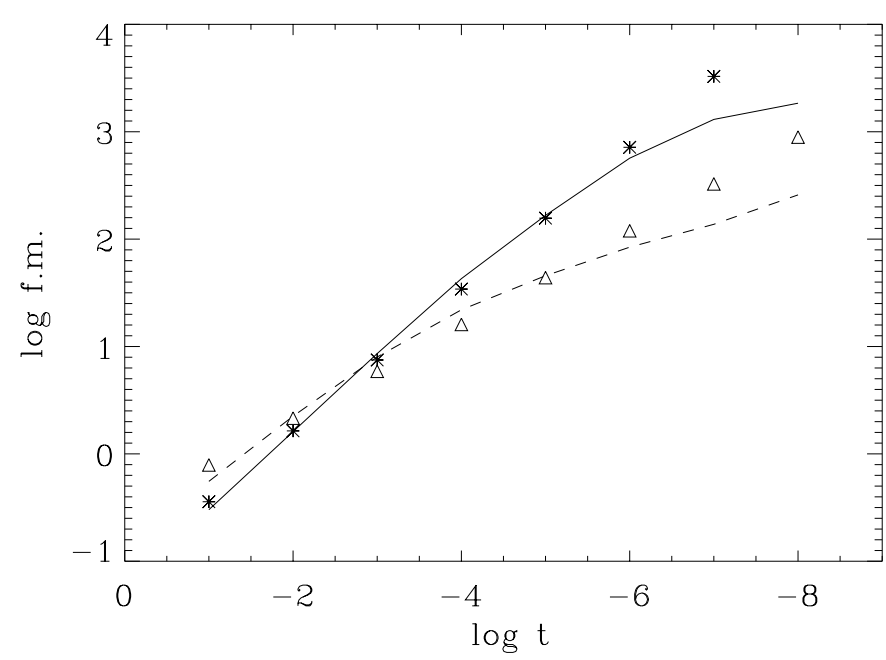

Fig. 2. Log force-multiplier as function of $\log t=-\log k_{1}$, for the models with $T_{\text {eff }}=50000 \mathrm{~K}$ (fully drawn) and $10000 \mathrm{~K}$ (dashed). For parameters, see Table 2. Asterisks and triangles gives linear regression for $\hat{\alpha}$ in the range $\log t=-1 \ldots-6$

appropriate Kurucz fluxes will change only some quantitative, however not qualitative conclusions, cf. Sect. 4.2.8). The local slope of this distribution, in the log-log representation, then corresponds to $\alpha-1$.

Force-multipliers are calculated by explicitly summing up the individual components (Eq. 17) as function of given depth parameters $t=k_{1}^{-1}$ and normalizing to the Thomson acceleration. If we are interested also in $\hat{\delta}$, the whole procedure is repeated for different values of $n_{\mathrm{e}} / W$ controlling the ionization/excitation balance ${ }^{3} . \hat{\alpha}$ and $\hat{\delta}$ are then found from local logarithmic derivatives with respect to $t$ and $n_{\mathrm{e} 11} / W$, where $n_{\mathrm{e} 11}$ is the electron density in units of $10^{11} \mathrm{~cm}^{-3}$.

Typical examples for the total variation of $\hat{\alpha}$ and $\hat{\delta}$ are given in Sect. 4, here we will constrain ourselves to the case of a fixed value of $n_{\mathrm{e} 11} / W=10, W=0.33$ and various effective temperatures in the range between $50000 \ldots 10000 \mathrm{~K}$.

\footnotetext{
${ }^{3}$ So far, we have not been concerned with $\hat{\delta}$. Before discussing this quantity in Sect. 4.1, we anticipate that the variation of $n_{\mathrm{e}} / W$ (for given $t$ ) leads to a variation in total line number $\propto N_{\mathrm{o}}$, which can be described by $N_{\mathrm{o}}\left(n_{\mathrm{e} 11} / W\right)^{\hat{\delta}}$.
} 


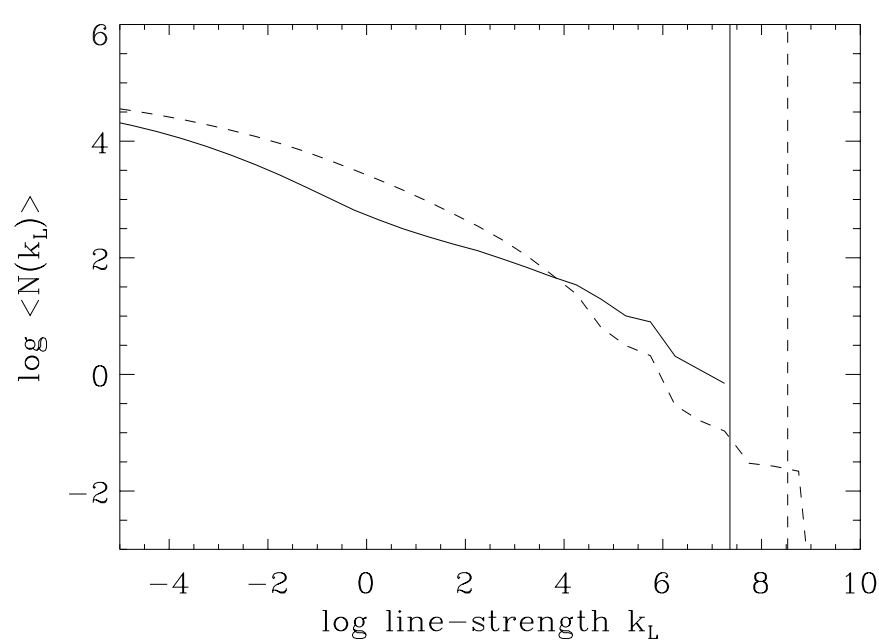

Fig. 3. (Planck-)Flux weighted cumulative line-strength distribution function, for the models with $T_{\text {eff }}=50000 \mathrm{~K}$ (fully drawn) and $10000 \mathrm{~K}$ (dashed). For parameters, see Table 2. Vertical lines give line-strengths corresponding to $Q_{\mathrm{o}}$ (calculated from $\bar{Q}, k_{\mathrm{CAK}}, \alpha$, cf. Eq. (38)) for the appropriate model. Note, that the cutoff of the distribution functions lies exactly at the calculated value!

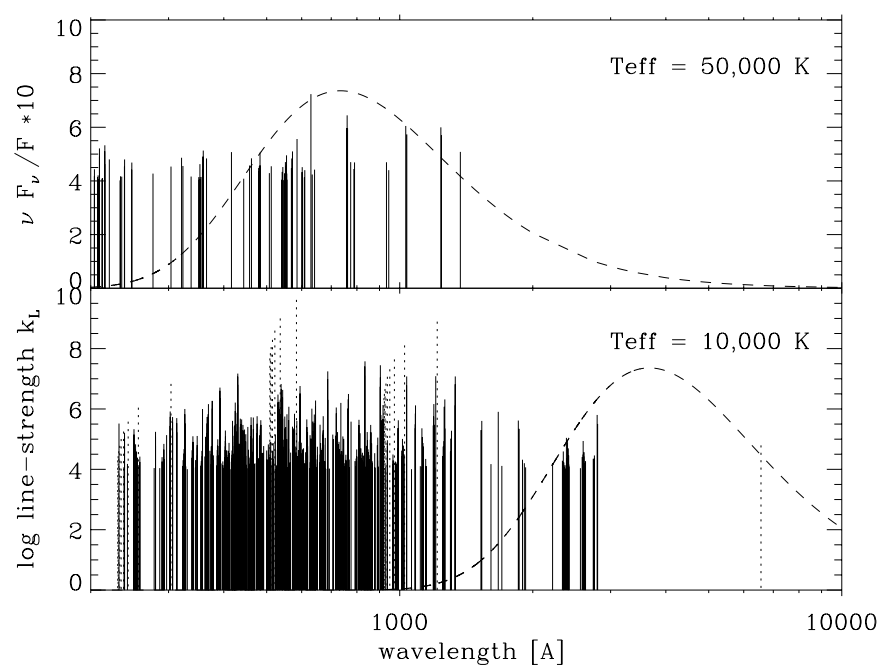

Fig. 4. Frequential distribution of the strongest lines $\left(\log k_{\mathrm{L}} \geq\right.$ 4) for the models of Fig. 3, in the range 200 to $10000 \AA$. Lines from hydrogen and helium are indicated by dots. Overplotted is the frequential weight factor $\nu F_{\nu} / F$, magnified by a factor 10

At first, let us concentrate on the force-multipliers. Table 2 gives the values of $k_{\mathrm{CAK}}$ and $\hat{\alpha}$ as function of temperature, which in this case were calculated by a linear regression $\log$ f.m. versus $\log t$ for the range $\log t=$ $-1 \ldots-6$. Figure 2 shows the corresponding function for the borders of our temperature range. The behaviour is rather monotonic: $k_{\mathrm{CAK}}$ increases with decreasing temperature, indicating an increasing potential of flux-blocking, and $\hat{\alpha}$ decreases from the canonical value $2 / 3$ to 0.44 at the lowest temperature. Note, that the actual forcemultiplier shows an almost exactly constant slope in the hot wind case, whereas for the cool temperature a curvature is present.

\subsection{Validity of Gayley's Ansatz}

For the models displayed, we have calculated $\bar{Q}$ from Eq. (37), and, by comparing with the corresponding value of $k_{\mathrm{CAK}}$, derived the $Q_{\mathrm{o}}$ value implied by (38). At first note that $\bar{Q}$ lies exactly in the range given by Gayley, and that especially at the hotter temperatures the favourized value of 2000 is exactly met. Second, $\bar{Q}$ decreases to lower temperatures, again in concert with the findings by Gayley. However, it is also obvious, that the (power-law) "equality" $Q_{\mathrm{o}}=\bar{Q}$ (Gayley's Ansatz!) is only met by the hotter models, whereas for the cooler ones a mismatch beyond a factor of ten is present.

The last panel in Table 2 gives the resulting $k_{\mathrm{CAK}^{-}}$ value if $Q_{\mathrm{o}}=\bar{Q}$ actually would have been set. Clearly, this assumption leads to much too large $k_{\mathrm{CAK}}$ 's, or, in other words, the estimated mass-loss rates would be much too high!

Let us firstly check whether the $Q_{\mathrm{o}}$-values derived from our line-force parameterization (38) and $\bar{Q}$ have anything to do with reality. For this reason, Fig. 3 displays the corresponding line-strength distribution functions, fluxweighted and cumulative. At first note the strong correspondence with the force-multiplier plot from above. For the hot wind, the slope is almost constant, which is the final reason that also the f.m. plot displays this behaviour, as explained in Sect. 2. In contrast, for the cooler temperature the distribution is curved, and the transition point between a rather steep (low $\alpha$ ) and a flatter slope is located at the same line-strength as in the f.m. plot, namely at $\log k_{\mathrm{L}}=4$ corresponding to $\log t=-4$.

We have indicated the calculated values of $Q_{\text {o }}$ (translated to $k_{\mathrm{L}}$ ) by vertical lines. Obviously, they have the correct order of magnitude, which is also true for the other three models which are not displayed. This result tells us that at least globally the assumption of a power-law distribution (required to validate Eq. (38)) seems to be justified, although the precise numbers (which are important for quantitative predictions since $\dot{M} / L \propto Q_{\mathrm{o}}^{-1}$ if $\left.Q_{\mathrm{o}} \neq \bar{Q}\right)$ depend on the curvature of the distribution, of course.

Since we have displayed the flux (times frequency) weighted distribution function required to calculate lineforces, the value of $\left\langle N\left(k_{\max }\right)\right\rangle$ gives some information about the frequential position of the strongest line(s). Whereas for the hotter atmosphere this number is close to unity (i.e., the strongest lines are close to flux maximum), the significantly lower value for the cooler atmosphere immediately points to the fact that here the strongest lines are disconnected from the maximum.

This obviously increasing mismatch between the position of the strongest lines and the flux-maximum is, besides the discussed influence of curvature terms, the primary reason for the "observed" difference between $\bar{Q}$ and 


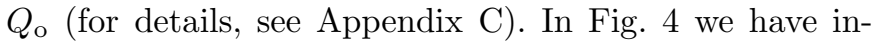
dicated the frequential line distribution for all lines with $\log k_{\mathrm{L}}>4$, overplotted by the according flux weighting factor $L_{\nu_{\mathrm{i}}} \nu_{\mathrm{i}} / L$ (magnified by a factor of ten for convenience). In accordance with the previous figure, the lines for the hotter model are almost uniformly distributed over the total contributing frequential regime. The cooler one, however, has its maximum density of strong lines in the Wien-regime of the radiation field ${ }^{4}$. This behaviour bases on the fact that (for all temperatures and "normal" composition) the strongest lines (excluding $\mathrm{H} / \mathrm{He}$ ) are the resonance lines of the CNO-group (Sect. 4.2.6) which are located, independently of ionization, in the UV. (E.g., the positions of the (second strongest) CII and OVI resonance lines at $\sim 1030 \AA$ are almost identical.) In consequence, the average weight factor of the strongest lines which dominate $\bar{Q}$ is decreasing for decreasing temperature and leads, as discussed in Appendix $\mathrm{C}$, to an increasing ratio of $Q_{\mathrm{o}} / \bar{Q}$.

In conclusion, our comparison has shown that the principle formalism provided by Gayley is valid to the same degree of precision than the older CAK parameterization. At least for the cooler stars, however, one has to account for the presence of an average $\bar{Q}$ (much) smaller than the maximum line-strength $Q_{\mathrm{o}}$. In so far, the problem of a rather unpredictable behaviour of $k_{\mathrm{CAK}}$ (if one has no tool to calculate it) is replaced by the simultaneously unknown ratio of $\bar{Q} / Q_{\mathrm{o}}$. Only in cases when the frequential distribution is uniform and the line-strength distribution has a constant slope, $Q_{\mathrm{o}}=\bar{Q}$ can be set. Thus, only simple cases (hot Supergiant winds) can be treated by the simple version of the formalism, whereas in all other cases (thin, metal-poor or cooler winds) at least one of the above problems prevents a blind application.

\section{What determines the slope?}

Having calculated the (flux weighted) line-strength distribution and appropriate force-multipliers as function of local variables, one could argue that the problem is solved, since, after performing the required regressions with respect to $\hat{\alpha}$ and $\hat{\delta}$ (see Kudritzki et al. 1998), these values can be tabulated and the hydro-equations solved. In the following, we want to proceed a step further and try to understand the basic physics which determines the slope of the distribution in some detail. Note again that the actual value of this quantity is decisive for all astrophysical problems involving radiatively driven mass-loss (cf. Sect. 1), and that only a thorough understanding of the individual processes which determine this quantity enables us to draw correct conclusions and to give quantitatively correct predictions. Moreover and although it is

\footnotetext{
${ }^{4}$ We note that the inclusion or neglect of the very strong, however somewhat peculiar (Sect. 4.2.6) hydrogen Lyman lines does not affect the discussed discrepancy.
}

empirically known that $\alpha$ is of order $0.4 \ldots 0.6$ in most situations (leading to the aforementioned similarity of $\alpha$ and $\hat{\alpha}$ ), there is no a priori reason that the steepness of the line-strength distribution function lies in this range, and, especially, that $\alpha>0$ over a large range of line-strengths.

From the definition of the flux-weighted line-strength distribution, there are three essential quantities to be considered in order to predict its behaviour, namely the oscillator strength distribution of contributing lines, the level population of the absorbing/re-emitting levels and the irradiating flux. In a first and more tutorial step, we consider the most simple case being possible, namely the case of pure hydrogen (or, more generally, hydrogenic ions), which states the complete problem (including the influence of NLTE-effects) in an analytically understandable way and leads to a number of interesting results.

\section{1. gf-and line-strength distribution for hydrogenic ions}

\subsection{1. $g$-distribution}

The $g f$-value of a given transition between principal quantum number $n^{\prime}$ and $n$ (summed over all contributing angular momenta, i.e. accounting for selection rules) is given by the well known Kramers formula (neglecting Gauntfactors of order unity)

$g f\left(n^{\prime}, n\right)=\frac{64}{3 \pi \sqrt{3}}\left(\frac{1}{n^{\prime 2}}-\frac{1}{n^{2}}\right)^{-3}\left(\frac{1}{n^{3} n^{\prime 3}}\right)$.

At first, consider only resonance transitions $n^{\prime}=1$, in which case $g f(1, n) \approx C / n^{3}$ with $C$ the numerical constant in (44). The number of possible transitions up to a certain principal quantum number $n$ is

$N(n)=n-1 \approx\left(\frac{C}{g f(1, n)}\right)^{\frac{1}{3}}-1$,

so that the (cumulative) number of transitions with $g f$ values stronger/equal than a certain value $g f$ reads

$N(g f)=\left(\frac{g f}{C}\right)^{-\frac{1}{3}}-1$

Thus, the resulting distribution function

$\frac{\mathrm{d} N}{\mathrm{~d} g f} \propto-g f^{-\frac{4}{3}}$

is given by a power-law, where the exponent corresponds to an $\alpha$-value of $2 / 3$, i.e., is just the canonical value which would lead to a mass-independent WLR and is consistent with the observations of O-Supergiants (cf. Puls et al. 1996).

Thus, from the above arguments one might conclude that the major problem is solved, and that the calculated/observed $\alpha / \hat{\alpha}$ values are dominated by the oscillator-strength statistics. Note already here, however, that the majority of driving lines (non-hydrogenic!) cannot be described similarily, since the dominant ingredient 


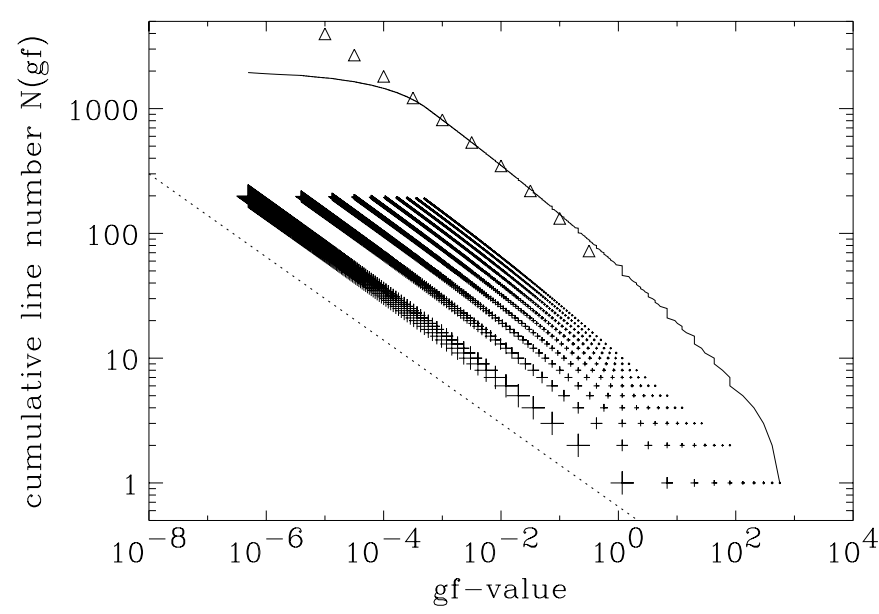

Fig. 5. $g f$-distribution for hydrogen, calculated by Kramersformula (Eq. 44) for $n^{\prime} \leq 10$ and $n \leq 200$. Fully drawn: Lines from all series with $n^{\prime} \leq 10$; dotted: Reference line with constant slope of $-1 / 3$ (with respect to $\log -\log$ ). Crosses: Different series, starting with Lyman series (leftmost). Size of crosses decreases with increasing $n^{\prime}$. Triangles: analytic approximation for small $g f$-values, Eq. (49)

in the above derivation - the rather simple and specific dependency of atomic quantities (oscillator strength and energy levels) on principal quantum number - is no longer valid in more complex ions.

Two additional points are worth mentioning. First, by using the "exact" Kramers law accounting for the $\left(1-n^{-2}\right)$ term, the apparent slope at large $g f$-values becomes steeper than $-4 / 3$, leading to $\alpha<2 / 3$, which again is consistent with the behaviour of "realistic" line-strength distribution function.

Second, by accounting also for transitions between excited levels, one finds (see below) the same statistics, i.e., the exponent $-4 / 3$ (with lower values for large $g f$ ) is universal for hydrogen-like ions.

Figure 5 displays the cumulative $g f$-distribution function for principal quantum numbers $n^{\prime} \leq 10$ (with sufficiently large $n \leq 200)$, both for the individual series $\left(n^{\prime}\right.$ fixed) denoted by crosses as well as for all combinations of $n^{\prime}, n$ (fully drawn). Following a steep increase from the maximum value, the distribution displays a rather constant slope (in the log) of $-1 / 3$ (cf. Eq. 46) over four dex, before it reaches its final, constant value resulting from running out of lines (depending on the maximum value chosen for $n$ ). This behaviour compares well to our case "A" discussed in Appendix A. Concerning the individual series, the higher ones follow exactly the predicted slope from the approximation given below (49), whereas the high $g f$-tails of the lower series have a somewhat steeper slope, as discussed above. Note also, that the $g f$-values from the higher series are generally larger than those from the lower ones.

A simple expansion of Eq. (44) clarifies the behaviour of the $g f$ distribution for not too large $g f$-values: To first

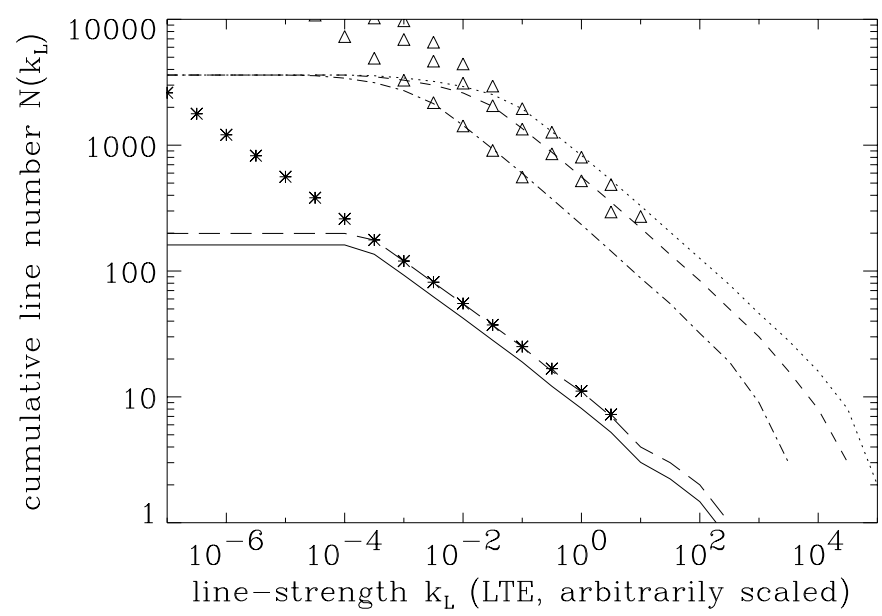

Fig. 6. Cumulative line-strength distribution function for hydrogen (see Eq. 50). "Atomic model" as in Fig. 5, gf-values from Kramers-formula and excitation in LTE. Triangles: analytic approximation for small $k_{\mathrm{L}}$; dotted: $T_{\mathrm{e}}=40000 \mathrm{~K}$; short dashes: $T_{\mathrm{e}}=30000 \mathrm{~K}$; dashed-dotted: $T_{\mathrm{e}}=20000 \mathrm{~K}$. Long dashes and asterisks: $T_{\mathrm{e}}=30000 \mathrm{~K}$, only Lyman series. Fully drawn: flux-weighted line distribution $(T=30000 \mathrm{~K})$, all lines, flux approximated by Planck-function

order, the upper level $n$ for given $g f$-value and lower level $n^{\prime}$ results to

$n\left(g f, n^{\prime}\right) / n^{\prime}=\left(\frac{g f}{C}\right)^{-\frac{1}{3}}+O\left(\frac{g f}{C}\right)^{\frac{1}{3}}$.

Thus, the total number of lines with $g f$-values larger than a certain one is given by

$$
\begin{aligned}
N(g f) & =\sum_{n^{\prime}=1}^{n_{\max }^{\prime}} n\left(g f, n^{\prime}\right)-n^{\prime}=\sum_{n^{\prime}=1}^{n_{\max }^{\prime}} n^{\prime}\left(\left(\frac{g f}{C}\right)^{-\frac{1}{3}}-1\right)= \\
& =\frac{n_{\max }^{\prime}\left(n_{\max }^{\prime}+1\right)}{2}\left[\left(\frac{g f}{C}\right)^{-\frac{1}{3}}-1\right], g f \ll C,(49)
\end{aligned}
$$

where $C$ is the numerical constant in Eq. (44) and $n_{\max }^{\prime}$ the maximum lower level of the considered transitions, which controls the vertical offset of the distribution function. Note, that the last equation (all transitions) compares exactly to Eq. (46) (only resonance lines), except from the generalization to $n_{\max }^{\prime}>1$.

\subsubsection{Line-strength distribution in LTE}

Although it is quite interesting that the $g f$-distribution follows an exponent of $-4 / 3$, our final interest is, of course, the behaviour of the line-strength distribution, i.e., the influence of excitation has to be accounted for. Figure 6 visualizes the corresponding function in the LTE approximation for three different temperatures, $T_{\mathrm{e}}=20000,30000$ and $40000 \mathrm{~K}$, as well as the following analytical result, 
which is derived in Appendix D:

$$
\begin{aligned}
N\left(k_{\mathrm{L}}, T_{\mathrm{e}}\right) & =k_{\mathrm{L}}^{-\frac{1}{3}}\left(C^{\prime} \mathrm{e}^{-\frac{h \nu_{1}}{k T_{\mathrm{e}}}}\right)^{\frac{1}{3}} f\left(T_{\mathrm{e}}\right)- \\
& -\frac{n_{\max }^{\prime}\left(n_{\text {max }}^{\prime}+1\right)}{2}, k_{\mathrm{L}}<C^{\prime} \mathrm{e}^{-\frac{h \nu_{1}}{k T_{\mathrm{e}}}} .
\end{aligned}
$$

(For the definition of $C^{\prime} \propto n_{1} / \rho$ with $n_{1}$ the ground-state occupation number and $f\left(T_{\mathrm{e}}\right)$, see Eq. D5.) Obviously, also the line-strength distribution follows the "canonical" power-law $\mathrm{d} N / \mathrm{d} k_{\mathrm{L}} \propto-k_{\mathrm{L}}^{-4 / 3}$, which is, of course, the final consequence of the primary dependence of $g f \propto n^{-3}$ for each series. Excitation plays only a minor role, controlling the function $f\left(T_{\mathrm{e}}\right)$ and thus the vertical offset (or, in other words, the normalization constant): the higher the temperature, the more lines are present if the ionization structure would remain constant, i.e., if $n_{1} / \rho$ would not change. Again, we have compared the analytical prediction with the numerical simulation (Fig. 6), which gives a perfect agreement in the valid range and even beyond! Note also, that the asymptotic behaviour $N\left(k_{\mathrm{L}}\right) \propto k_{\mathrm{L}}^{-1 / 3}$ is consistent with our requirement that $k_{\mathrm{L}}\left\langle N\left(k_{\mathrm{L}}\right)\right\rangle \rightarrow 0$ for $k_{\mathrm{L}} \rightarrow 0$, which has to be fulfilled in order to validate Eq. (21).

\subsubsection{Flux weighting}

The next question which arises in our simple example is that concerning the influence of flux-weighting. One might note, also in a more general context, that this "final operation" in our course of deriving radiative accelerations (enough irradiating photons have to be present!) leads to the actual possibility to perform quantitative simulations: Since we have seen, that the strongest $k_{\mathrm{L}}$ values arise from IR-transitions between excited levels (cf. the high linestrength end of Fig. 6), without a final flux-weighting we would have to account for atomic models which were by far too large to be treated numerically!

Fortunately, the flux irradiating these strong IRtransitions is small, and the primary contribution to the line-force is only due to transitions near the flux maximum, a fact which is exploited a priori in our simplified NLTE-calculations (cf. Sect. 3.1.2 and Abbott \& Lucy 1985). With respect to the hydrogen atom under consideration, we expect therefore the Lyman series (including the Balmer series for A-stars) to be the major contributor for radiative momentum. To this end, the long-dashed curve in Fig. 6 displays the line-strength distribution for the Lyman-Series only $\left(T_{\mathrm{e}}=30000 \mathrm{~K}\right)$, together with the analytical approximation (asterisks), whereas the fully drawn one corresponds to the final, flux (times frequency) weighted distribution accounting for all considered lines: Obviously, our expectation is met precisely. Note, however, that Fig. 6 serves only as a tutorial and overestimates the real situation by far, since due to line convergence near the ionization edge only a few lines $(n \lesssim 10)$ can be treated as individual ones, thus cutting the distribution function at this value.

\subsubsection{Influence of NLTE-effects: The parameter $\delta$}

So far, we were concerned only with the slope of the line-strength distribution and its relation to the line-force parameter $\hat{\alpha}$. Almost nothing has been said on the normalization constant $N_{\text {o }}$ (Eq. 10), which controls the absolute value of the acceleration due to its relation to the total line number. We have pointed out that $N_{\mathrm{o}}$ may have an additional depth dependence, which transforms, via Eq. (16), into a depth dependent line-force parameter $k_{\mathrm{CAK}}$. This additional depth dependence originates, of course, from the behaviour of the mass absorption coefficient $\bar{\chi}_{\mathrm{i}} / \rho$ present in the definition of the line-strength $k_{\mathrm{L}}$. Although this ratio remains more or less constant if we consider the primary source of radiative driving, namely resonance lines and lines with a lower meta-stable level from main ionization stages (denoted in the following by " $j ")$, there will be always a "contamination" by lines from excited levels and, most important, by lines from minor ionization stages.

Since HI is such a trace ion in hot star winds, we will investigate the effects of this depth dependence in our tutorial chapter. It is well known (e.g. Mihalas 1978, p. 125) that the NLTE ground state departure coefficient of a trace ion one stage below the major one in a spherical atmosphere is primarily a function of the inverse of the dilution factor

$W(r)=\frac{1}{2}\left[1-\left(1-\left(R_{*} / r\right)^{2}\right)^{\frac{1}{2}}\right]$.

One finds that

$n_{1, j-1} \sim \frac{n_{\mathrm{e}}}{W} n_{j} \Phi\left(T_{\mathrm{e}}\right)$,

if $n_{j}$ is the population of the dominant ion (here: HII), $\Phi$ the Saha-Boltzmann factor and the radiation temperature at the ionization edge is not too different from the electron temperature. Equation (51) simply states that in a spherical atmosphere the ground-state of ion $(j-1)$ becomes overpopulated $\propto 1 / W$, since the radiation field controlling the ionization is diluted with respect to the LTE-situation, whereas the recombination operates at its usual value. Note, that the above equation, with a couple of refinements, allows also for the approximate solution of the equations of statistical equilibrium in a spherical wind, as was described in Sect. 3.1.2.

Since $n_{j}$ varies in concert with the local density, the ratio $n_{1} / \rho$ becomes a function of $n_{\mathrm{e}} / W$, which finally leads - besides an additional temperature dependence - to a variation of $N_{\mathrm{o}}$ as (cf. Eq. 50)

$N_{\mathrm{o}} \propto\left(\frac{n_{\mathrm{e}}}{W}\right)^{\frac{1}{3}}$

In a mixture of major and minor ionization stages, as present in a stellar wind, we have, of course, a different 


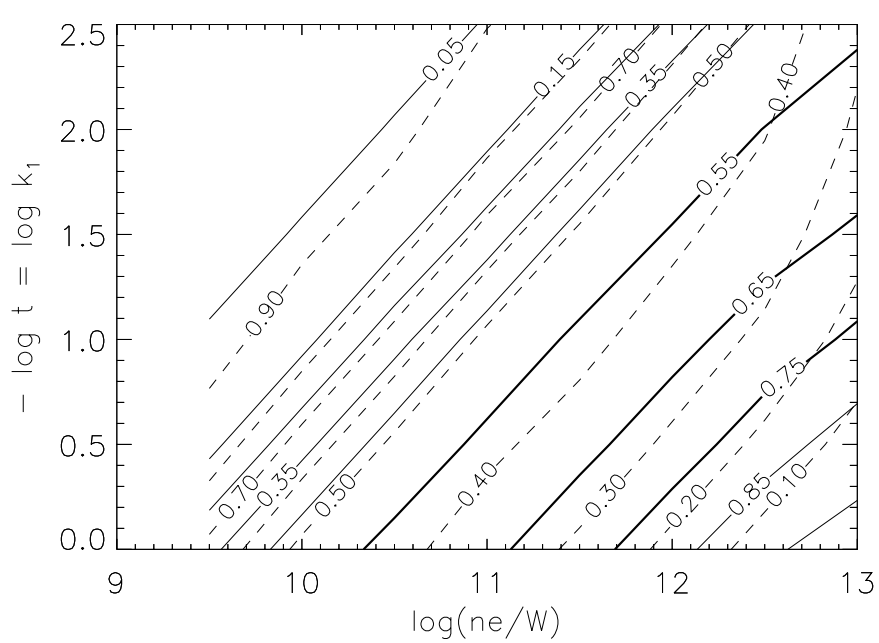

Fig. 7. Iso-contours of force-multiplier parameters $\hat{\alpha}$ and $\hat{\delta}$ as function of $\log \left(n_{\mathrm{e}} / W\right)$ and $-\log t=\log k_{1}$, for hydrogen with ten levels. $T_{\mathrm{e}}=40000 \mathrm{~K}$, dilution factor $W$ evaluated at $r / R_{*}=5 ., 4 ., 3 ., 2.5,2 ., 1.75,1.50,1.25$ and 1 , respectively. Fully drawn: $\hat{\alpha}$, dashed: $\hat{\delta}$. Thick curves enclose the region of $0.55<\hat{\alpha}<0.75$. A trajectory throughout a typical wind would stretch from the lower right corner to the upper left one

dependence. However, the notion that also in this case the ionization structure is primarily controlled by the factor $n_{\mathrm{e}} / W$ (with exponents different from unity if one includes trace ions of stages $j+1, j-2$ etc.) lead Abbott (1982) to the introduction of the famous $\delta$-term to the force-multiplier, which in terms of our line-strength distribution function reads

$\mathrm{d} N\left(\nu, k_{\mathrm{L}}, r\right)=-N_{\mathrm{o}}\left(\frac{n_{\mathrm{e} 11}}{W}\right)^{\delta} f\left(\nu, k_{\mathrm{L}}\right) \mathrm{d} \nu \mathrm{d} k_{\mathrm{L}}$,

with $n_{\mathrm{e} 11}$ the electron density in units of $10^{11} \mathrm{~cm}^{-3}$ and

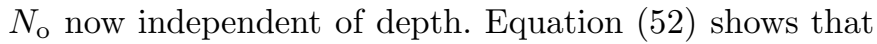
for hydrogen ${ }^{5} \delta$ should be of order $1 / 3$. More generally and using the fact that the appropriately scaled variable for all considerations is $\tilde{k} \propto k_{\mathrm{L}} / C^{\prime}$ (Eq. D2), a plasma dominated by trace-ions of stage $j-1$ must have a $\delta$-value which is just the negative of the line-strength exponent in the $N\left(k_{\mathrm{L}}\right)$-distribution, i.e, $\delta=-(\alpha-1)$ or

$\alpha+\delta=1$, trace ions of stage $(j-1)$ only!

Figure 7 verifies this equation for a number of different conditions. We have calculated the force-multiplier arising from hydrogen in a hot stellar wind plasma under different conditions (see caption), and obtained the effective values $\hat{\alpha}, \hat{\delta}$ by first order derivatives. Although $\hat{\alpha}$ varies from 0 $\ldots 1$, the equality (54) is almost perfectly reproduced. On a first glance, it is somewhat puzzling that $\hat{\alpha}$ is so much varying although the according line-strength distribution has an almost constant slope. In the situation described here (trace ion of stage $(j-1)$ ), however, we have a strong dependence on local conditions, $k_{\mathrm{L}} \propto \rho / W$, contrasted to

\footnotetext{
${ }^{5}$ If $\mathrm{HI}$ is a trace ion.
}

the usual case of $k_{\mathrm{L}} \approx$ const throughout the wind for the (resonance) lines of major ion species. Thus, there is only a small strip in the $k_{1},\left(n_{\mathrm{e}} / W\right)$ plane where we do not encounter the case of all lines being either optically thick $(\hat{\alpha} \rightarrow 1)$ or optically thin $(\hat{\alpha} \rightarrow 0)$. Even in those cases, however, Eq. (54) is still valid!

Finally, $\hat{\alpha}=1$ and $\hat{\delta}=0$ holds generally (i.e., for any type of ionization/excitation) in the optically thick case, since then the dependence of line-force on occupation numbers vanishes completely and only the relation $g_{\text {rad }}^{\text {tot }} \propto k_{1}\left\langle N_{\text {thick }}\right\rangle$ survives.

We want to finish this section with two summarizing comments: First, we have shown that for hydrogen (generally: hydrogenic ions) the $g f$-distribution $g f\left(n^{\prime}, n\right) \propto n^{-3}$ (for each series) leads quite naturally to a line-strength distribution function with a slope corresponding to $\alpha \approx$ $2 / 3$. Second, the dependence of the line-strength on the specific type of ionization (major one or trace ion) gives rise to a depth dependent normalization constant $N_{\text {o }}$, finally leading to the $\delta$ term in the force-multiplier and linestrength statistics. Since $\delta$ is of order $1 / 3$ for trace ions of stage $j-1$ (which are usually the dominating species of minor ions, cf. Sect. 4.2.5) and $\delta=0$ for major ions, the effective value of $\delta$ resulting from an appropriate mixture should be a small, positive number ${ }^{6}$.

\subsection{Arbitrary ions}

Since the predominant radiative acceleration in hot star winds is certainly due to non-hydrogenic ions (e.g., from iron-group elements), we turn now to the line-strength statistics of these elements.

To our knowledge, there are only two previous investigations related to this topic. On the one hand, Learner (1982) found a line-strength distribution with a typical power-law index corresponding to $\alpha=0.5$ by means of experimental data (mostly ionization stages I and II), a result recently used by Wehrse et al. (1998) in their stochastic approach of line transfer in moving atmospheres. The first step, however, was taken by Allen $(1966,1974)$ who performed a thorough analysis of line distributions of neutral elements, aimed at the goal of establishing a "statistical spectroscopy". His approach provides a significant understanding of the resulting statistical description especially under LTE conditions. The basic philosophy, however, has turned out to be extremely useful also regarding the problem under our consideration, at least if some additional considerations are taken into account. Before we comment on these, we will firstly introduce the basic concept and convince ourselves that it is applicable for our purposes. Note that the following results, which allow to understand the line-strength statistics of

\footnotetext{
${ }^{6}$ Under very specific conditions, the effective $\hat{\delta}$ can even become negative.
} 
individual ions in an almost completely analytical way (as a function of level density, oscillator-strength distribution, ionization potential, temperature etc.), will turn out to be useful also for future investigations related to lineblanketing/blocking calculations. Finally, by a comparison between actual data and analytic simulations based on our results, the degree of completeness of present atomic data bases can be easily checked.

\subsubsection{Allen's approach}

Following Allen $(1966,1974)$ and neglecting subtleties, the first important assumption concerns the number of energy levels in the energy range $x, x+\mathrm{d} x$, which will be described by the distribution $p(x) \mathrm{d} x$. Although there are certain irregularities, the basic trend of $p(x)$ is to increase with excitation energy, and Allen adopted - per ion - a power-law

$p(x$, per $\mathrm{d} x)=a 10^{x / \sigma}$

in the range $0<x<i_{\mathrm{e}}$ with (effective) ionization energy $i_{\mathrm{e}}$. Note that this parameterization can be validated for rather complex ions (see the tests performed below), is however much less appropriate for hydrogenic ions due to their specific dependence of energy on level number (cf. the examples given by Allen 1966, Fig. 3). Thus, the approach presented here and the one given in the previous section are almost mutual exclusive.

With the above distribution, the number of lines between energies $x_{1}$ and $x_{2}$ and per excitation ranges $\mathrm{d} x_{1}$ and $\mathrm{d} x_{2}$ is

$\Delta N\left(x_{1}, x_{2}\right.$, per $\left.\mathrm{d} x_{1}, \mathrm{~d} x_{2}\right)=j p\left(x_{1}\right) p\left(x_{2}\right)$,

where $j \leq 1$ shall be the "selection" factor accounting for selection rules (typically of order $0.2 \ldots 0.3$ for iron group elements). Since the transition frequency is given by $\nu=x_{2}-x_{1}$, when we calculate in appropriate units (say, kiloKayser $=$ "kK"), the number of lines with lower levels between $x_{1}, x_{1}+\mathrm{d} x_{1}$ and transition frequencies $\nu, \nu+\mathrm{d} \nu$ is

$\Delta N\left(x_{1}, \nu\right.$, per $\left.\mathrm{d} x_{1}, \mathrm{~d} \nu\right)=j a^{2} 10^{\frac{2 x_{1}+\nu}{\sigma}}$.

In his further derivation, Allen assumed the logarithm of the $g f$-values $(\log g f=: r)$ to be equally distributed within the range $r_{\max }>r>r_{\min }$, with $r_{\max }-r_{\min }$ of order 3 dex. This assumption, being equivalent to a $g f$ distribution $|\mathrm{d} N / \mathrm{d} g f| \propto g f^{-1}$, will be relaxed in the following. Instead, we consider a distribution with arbitrary, however constant exponent $\gamma$, again independent of frequency (and excitation energy), so that the number of lines with $\log g f$-values within $r, r+\mathrm{d} r$ is given by

$\Delta N\left(x_{1}, \nu, r, \operatorname{per} \mathrm{d} x_{1}, \mathrm{~d} \nu, \mathrm{d} r\right)=\frac{j a^{2}}{w} 10^{\frac{2 x_{1}+\nu}{\sigma}+r(1-\gamma)}$, where $w$ is the normalization constant with respect to the adopted $g f$-statistics,

$w=\frac{10^{r_{\max }(1-\gamma)}-10^{r_{\min }(1-\gamma)}}{(1-\gamma) \ln 10}, \gamma \neq 1$

$w=r_{\max }-r_{\min }, \quad \gamma=1$.

Now we define the line intensity by

$l=-\log \left[g f \exp \left(-\frac{1000 x_{1} h c}{k T}\right)\right]=\frac{x_{1}}{t}-r$

if we measure energies and frequencies in $\mathrm{kK}$ and the temperature in units of $625 \mathrm{~K}, t=T / 625 \mathrm{~K}$ (not to be confused with the optical depth parameter defined by CAK).

Note that by introducing the line intensity in this way, one implicitly assumes that the plasma is in LTE and that all levels connected by lines play an equally important role, if one uses this quantity as the primary statistical variable. Note also that under LTE conditions $l$ is closely related to the negative logarithmic line-strength if the wavelength dependence of $k_{\mathrm{L}}$ is ignored.

If we convert the $x_{1}$-dependence of $\Delta N$ into an $l$-dependence and integrate over $\mathrm{d} r$, we find the number of lines for given line intensity and transition frequency as

$$
\begin{aligned}
\Delta N & (l, \nu, \text { per } \mathrm{d} l, \mathrm{~d} \nu)= \\
& =\frac{j a^{2} t}{w} 10^{\frac{2 l t+\nu}{\sigma}} \int_{r_{\min }}^{\tilde{r}_{\max }} 10^{\left(\frac{2 t}{\sigma}+1-\gamma\right) r} \mathrm{~d} r= \\
& =\frac{j a^{2} t}{A w \ln 10} 10^{\frac{2 l t+\nu}{\sigma}}\left(10^{A \tilde{r}_{\max }}-10^{A r_{\min }}\right) \\
A & =\left(\frac{2 t}{\sigma}+1-\gamma\right)
\end{aligned}
$$

(Here and in the following, we always assume $A \neq 0$. The case $A=0$ can be treated by a somewhat different expansion). In case of $\gamma=1$, the second term in the difference can be neglected with respect to the first one, and we recover the result given by Allen,

$\Delta N(l, \nu$, per $\mathrm{d} l, \mathrm{~d} \nu) \stackrel{\gamma=1}{=} \frac{j a^{2} \sigma}{2 w \ln 10} 10^{\frac{2 t}{\sigma}\left(l+\tilde{r}_{\max }\right)+\frac{\nu}{\sigma}}$

The difference between $\tilde{r}_{\max }$ and $r_{\max }$ is the following. Whereas $r_{\max }$ relates to the maximum $\log g f$-value for the considered ion, $\tilde{r}_{\max }$ is the maximum value which is possible for given $l$ and $\nu$ and underlies the following restrictions:

$$
\begin{aligned}
r & <r_{\max }=-l_{\min } \\
x_{1} & +\nu<i_{\mathrm{e}}, \text { i.e. } \\
r & <\frac{i_{\mathrm{e}}-\nu}{t}-l \\
\rightarrow \tilde{r}_{\max } & =\operatorname{Min}\left(\frac{i_{\mathrm{e}}-\nu}{t}-l,-l_{\min }\right),
\end{aligned}
$$

where the transition occurs at

$l_{\mathrm{T}}=\frac{i_{\mathrm{e}}-\nu}{t}+l_{\min }$. 
Let us first consider the implications of this restriction in the case of $\gamma=1$. In the following, the line-number $\Delta N$ is always to be understood as function of $(l, \nu)$ per interval $\mathrm{d} l$ and $\mathrm{d} \nu$.

$$
\begin{aligned}
& \log \Delta N \stackrel{l<l_{\mathrm{T}, \gamma=1}}{=} \log \frac{j a^{2} \sigma}{2 w \ln 10}+\frac{2 t}{\sigma}\left(l-l_{\min }\right)+\frac{\nu}{\sigma} \\
& \log \Delta N \stackrel{l \geq l_{\mathrm{T}, \gamma=1}=1}{=} \log \frac{j a^{2} \sigma}{2 w \ln 10}+\frac{2 i_{\mathrm{e}}}{\sigma}-\frac{\nu}{\sigma} .
\end{aligned}
$$

The interpretation of these expressions is straightforward. If the $r$-values are equally distributed between $r_{\max }$ and $r_{\text {min }}$, the $\log$ of $\Delta N$ should increase linearly between $l_{\min } \ldots l_{\mathrm{T}}$, where the slope is controlled both by the slope of the level density and the temperature. For all line intensities larger than $l_{\mathrm{T}}$, the number of lines should become constant, until $\tilde{r}_{\max } \approx r_{\min }$ and the number of lines approaches zero for $l>\left(i_{\mathrm{e}}-\nu\right) / t-r_{\min }$.

In order to check this and the following predictions, we have calculated the line intensity statistics for FeII (comprising roughly 200000 lines) from our present data base (Sect. 3.1). Instead of using the actual $g f$-values, however, we firstly simulated different distributions by a MonteCarlo process, with random variable $x \in(0,1]$,

$$
\begin{gathered}
x=\frac{\int_{g f}^{g f_{\max }} g f^{-\gamma} \mathrm{d} g f}{\int_{g f_{\min }}^{g f_{\max }} g f^{-\gamma} \mathrm{d} g f} \\
g f(x) \stackrel{\gamma \neq 1}{=} g f_{\max }\left[1-x\left(1-\left(\frac{g f_{\min }}{g f_{\max }}\right)^{1-\gamma}\right)\right]^{\frac{1}{1-\gamma}}, \\
g f(x) \stackrel{\gamma=1}{=} 10^{r_{\max }}+x\left(r_{\min }-r_{\max }\right) .
\end{gathered}
$$

and replaced the actual $g f$-value by the value drawn from the above distribution, with $g f_{\max }=1$ and $g f_{\min }=10^{-7}$. Thus, by this simulation we primarily investigate in how far the assumptions leading to Eq. (57) are justified and inspect the validity of the overall approach, under the restriction of $g f$-distributions with constant slope.

In the following plots, we display the result for the frequency with the highest line-density, namely $\nu=65.5 \pm$ $2.5 \mathrm{kK}$ corresponding to $\lambda=1538 \ldots 1667 \AA$, and the vertical line gives the transition line intensity $l_{\mathrm{T}}$, with $i_{\mathrm{e}} \approx 130 \mathrm{kK}$.

The first series (Fig. 8) has been calculated for the case discussed above, namely $\gamma=1$, and three different temperatures $T=5000,10000,15000 \mathrm{~K}$. As predicted, the constant, temperature dependent slope and the kink at $l_{\mathrm{T}}$ are present, as well as the constant line number for $l>l_{\mathrm{T}}$, until the maximum possible $l$ (as function of $t$, see above) is reached and $\Delta N \rightarrow 0$.

Next, we consider the case of $\gamma \neq 1$. Here, we have to perform the following distinction. Let us first assume that $\left(\frac{2 t}{\sigma}+1-\gamma\right)$ is not significantly smaller than $2 t / \sigma$, i.e., $\gamma \lesssim 1.15$ in the case of FeII. Then, we can neglect again the $2 \mathrm{nd}$ term in the difference in Eq. (61) with respect to the first one, and obtain, accounting for the restrictions concerning $\tilde{r}_{\max }$,

$$
\begin{aligned}
& \log \Delta N \stackrel{l<l_{\mathrm{T}}}{=} \log \frac{j a^{2} \sigma}{\left(2+\frac{\sigma}{t}(1-\gamma)\right) w \ln 10}+\frac{2 t}{\sigma}\left(l-l_{\mathrm{min}}\right) \\
&+\frac{\nu}{\sigma}-(1-\gamma) l_{\min }, \quad\left(\gamma \lesssim 1+\frac{2 t}{\sigma}\right) \\
& \log \Delta N \stackrel{l \geq l_{\mathrm{T}}}{=} \log \frac{j a^{2} \sigma}{\left(2+\frac{\sigma}{t}(1-\gamma)\right) w \ln 10}-(1-\gamma) l \\
& \quad+\frac{2 i_{\mathrm{e}}}{\sigma}-\frac{\nu}{\sigma}+(1-\gamma) \frac{i_{\mathrm{e}}-\nu}{t}, \quad\left(\gamma \lesssim 1+\frac{2 t}{\sigma}\right)
\end{aligned}
$$

These equations are similar to the case $\gamma=1$ (Eqs. 66, 67), except from the offset and one decisive difference: For line intensities larger than $l_{\mathrm{T}}$, the line number is no longer constant, but becomes directly coupled to the oscillator strength statistics via the term $-(1-\gamma) l$. Thus, a declining line number is expected for $\gamma<1$, whereas for $\gamma$ (slightly) larger than unity the distribution function should increase. Note, that the predicted slope for $l>l_{\mathrm{T}}$ is independent of temperature!

Figure 9 impressively verifies our predictions. Here, we have simulated an oscillator strength distribution with $\gamma=.66$ (left panel), whereas in the middle one $\gamma=1.15$ was assumed. Note the abrupt change in the slope at $l \approx l_{\mathrm{T}}$.

Finally, we consider the case of a rather steep $g f$-distribution $\gamma>1+2 t / \sigma$, e.g. $\gamma \gtrsim 1.8$ for FeII. Then, the $g f$-distribution is dominated by its lower end, and the values taken at $r_{\min }$ are dominating both the normalization constant and the difference term, in contrast to the situation discussed above. Accordingly, $\tilde{r}_{\max }$ and $l_{\mathrm{T}}$ do no longer play any role, and we obtain a line number statistics

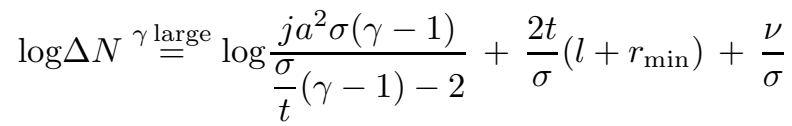

with uniform, temperature dependent slope $2 t / \sigma$. Note, that the only $\gamma$ dependence shows up in the offset. The right panel in Fig. 9 gives the corresponding result, for $\gamma=2$ and $r_{\min }=-3$, which was used in order to obtain a statistically significant number of lines at the high $g f$ end. Obviously, no kink is present any longer, and the distribution lies parallel to the low- $l$ part of a corresponding $(\gamma=1)$-distribution, overplotted as rectangles.

So far, our investigations can be summarized as follows. Allan's approach (extended for $\gamma \neq 1$ ) has been validated for a complex ion in those cases when $\gamma$ is constant, and the slope of the distribution function can be predicted: At low intensities (corresponding to high line-strengths), it is controlled both by the level density as a function of excitation energy (slope $\sigma$ ) as well as by the population of these levels (excitation temperature $t$ ), resulting from our LTE assumption. In this domain, the oscillator strength 

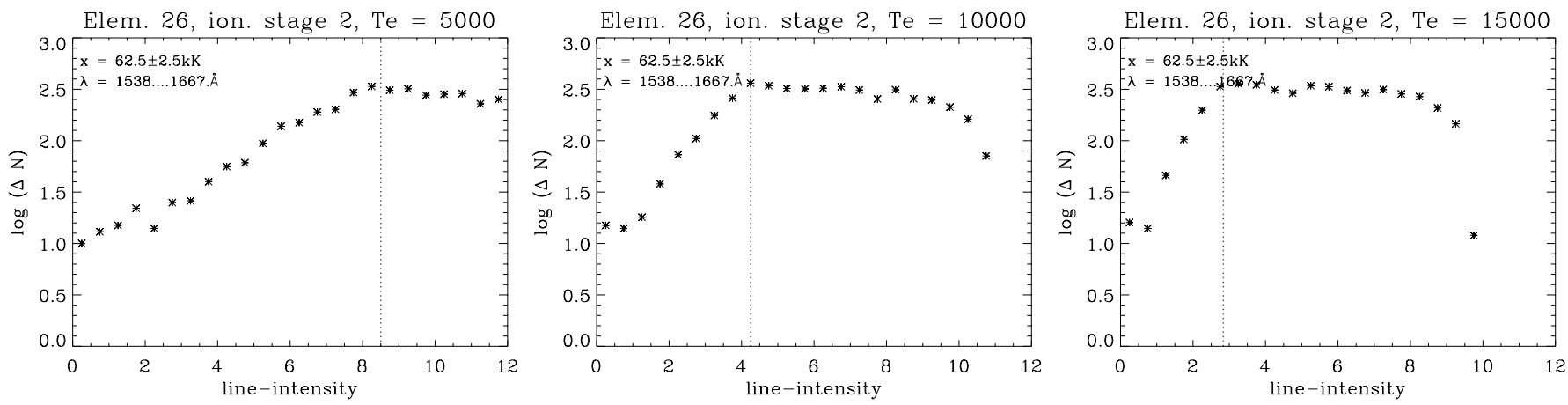

Fig. 8. $\Delta N$ statistics for FeII as function of line intensity, for three different temperatures (cf. text). Lines are counted per $5 \mathrm{kK}$ in frequency and per 0.5 dex in intensity. The vertical line displays $l_{\mathrm{T}}$ (Eq. 65). $g f$-distribution calculated by Monte-Carlo simulation adopting $\gamma=1$, cf. Eq. (69)
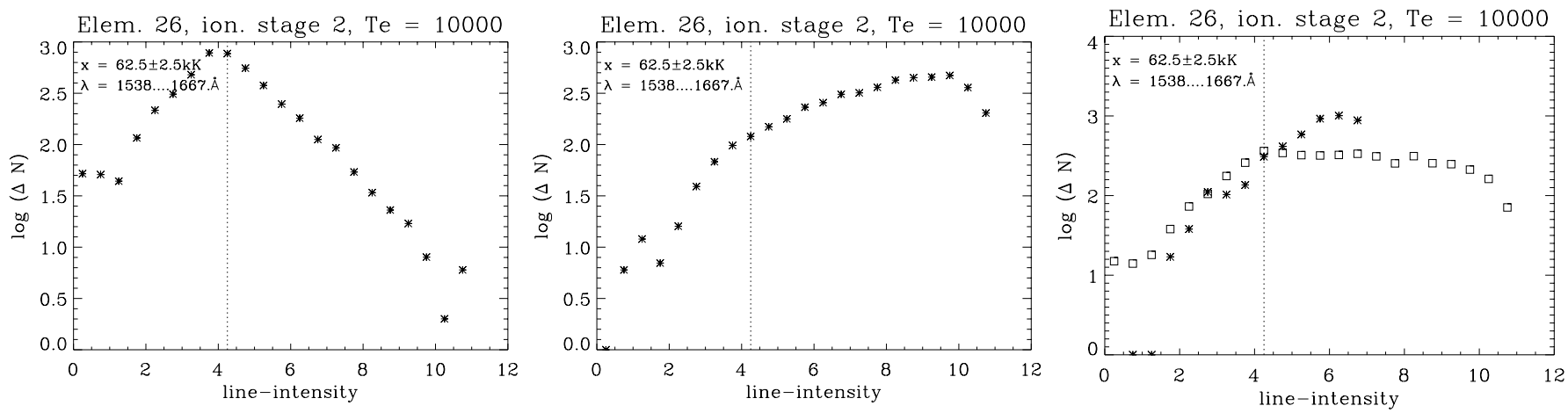

Fig. 9. As Fig. 8, for $T=10000 \mathrm{~K}$ and $\gamma=0.66$ (left), $\gamma=1.15$ (middle) and $\gamma=2.0$ (right). For the right panel, $\log g f_{\min }=-3$ was used, and the results of the corresponding $(\gamma=1)$-distribution are overplotted as rectangles

distribution seems to be of no importance for the slope, since - for $\gamma \lesssim 1+2 t / \sigma$ - the line number is primarily controlled by the highest $g f$-value, and for $\gamma>1+2 t / \sigma$ by the lowest one, both of which do "only" control the offset of the distribution. For large line intensities (weak lines!), we encounter a certain inter-relation between energetic neighbourhood to the ionization limit and maximum possible $g f$-value (cf. Eq. 64), which causes a slope dominated by the $g f$-distribution, provided $\gamma$ is not too large. In the opposite case, finally, the small $g f$-values become decisive over the complete range, and the slope of the distribution retains its previous slope for all $l$ values.

Thus, it seems that we have to know only the principal behaviour of the $g f$-distribution to predict the LTE line intensity/line-strength statistics for a certain ion. Unfortunately, Fig. 10 shows that this is at least not so simple. Here, we have plotted $\log \Delta N$ using the actual oscillator strengths. As is obvious, this distribution looks rather different from the cases discussed so far, primarily in the domain $l>l_{\mathrm{T}}$.

The reason for this different functional behaviour becomes evident from Fig. 11, displaying the actual run of the oscillator strength distribution in the considered frequency interval as well as for "all" lines of FeII and all lines (for the atoms $\mathrm{H}$ to $\mathrm{Zn}$, cf. Sect. 3.1) present in our data base.

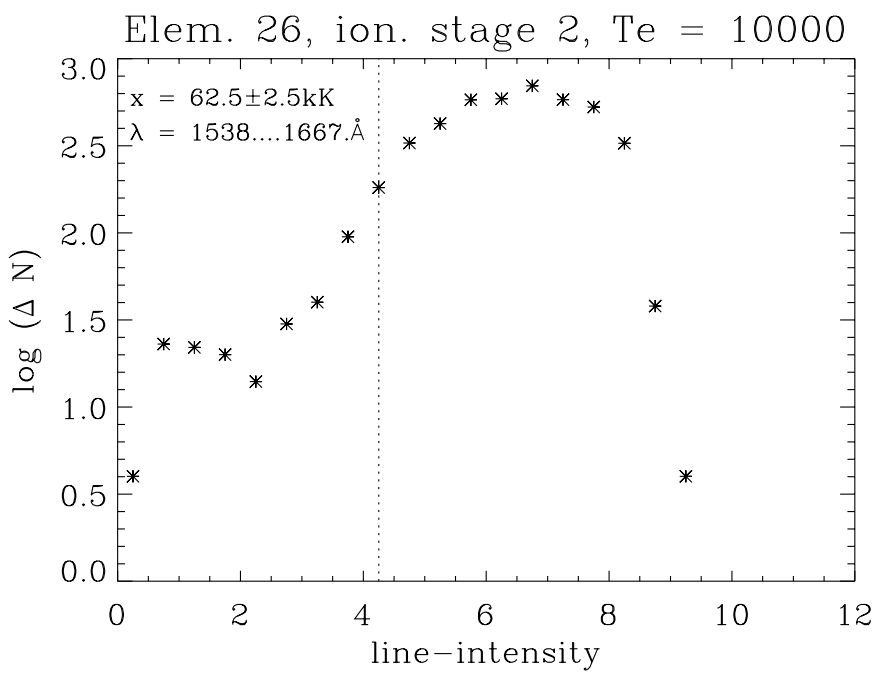

Fig. 10. As Fig. 9, for $T=10000 \mathrm{~K}$ and actual $g$-distribution

At first note that the specific shapes of the particular distributions are extremely similar, where the major differences concern the total line number $N\left(g f_{\min }\right)$ and the highest $g f$-value being present. In contrast to the case of hydrogenic ions, however, the distribution does no longer show a more or less constant slope, but is curved. At the high $g f$-end, the distribution is rather steep, with an 


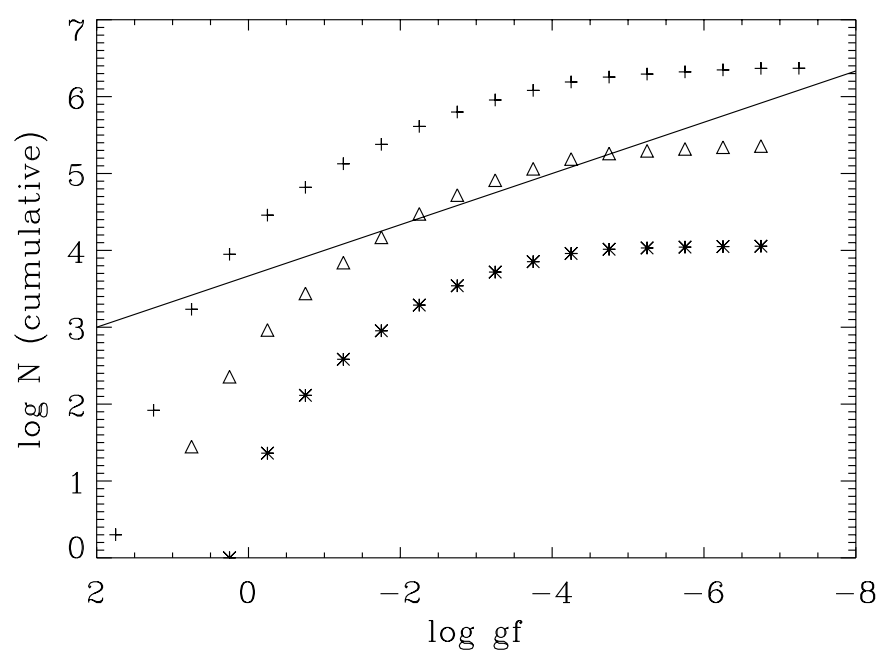

Fig. 11. Cumulative line number as function of logarithmic oscillator strength $r$. Asterisks: FeII, in the interval $\lambda=1538$ ...1667 $\AA$ (as in Fig. 8); triangles: FeII, all lines of our data base; crosses: all lines of elements $\mathrm{H}$ to $\mathrm{Zn}$ present in our data bases. For comparison, a reference line with constant slope (in the $\log$ ) of $-1 / 3$ (corresponding to $\gamma=4 / 3$ ) is indicated

approximate slope $\gamma \gtrsim 2$ over the first 2.5 dex, and may be described afterwards again by the canonical value $\gamma \approx 4 / 3$, before our line list becomes incomplete (for $\log g f<-5$ ). To our understanding, the steep increase results from the very strong lines connecting the (effective) ground states with low lying levels. These lines follow statistics different from the other ones, a feature which we have also found in case of hydrogenic ions (cf. also the related discussion in Allen 1966).

\subsubsection{Frequency integrated distribution functions}

Although the $g f$-distribution is significantly curved leading to certain subtleties in the intensity distribution if considered in a specified frequency range (as it was done, e.g., in Fig. 10), it might be suspected that on a larger average the description should become more uniform again. Note, e.g., that the largest part of the distribution function $(0 \gtrsim \log g f \gtrsim-4)$ can be described by a more or less constant slope, with a significantly steeper one only over the first two decades, which consequently comprise only few lines.

In order to check this hypothesis and to proceed towards our aim at describing flux-weighted line-strength distribution functions under stellar wind conditions, we will follow our analytical description assuming constant slope $\gamma$. From now on, however, we will concentrate on the distribution of "all" lines per ion, i.e., we will consider frequency integrated distribution functions. Most important to this regard is the frequency dependence of the threshold value $l_{\mathrm{T}}$ (Eq. 65), dividing the two different domains of influence, namely either by excitation and/or by $g f$-distribution. Since $l_{\mathrm{T}}$ decreases with frequency, lines with higher frequencies (transitions from the lowest levels, dominating the line-force) should be much more coupled to the oscillator strength distribution than lines with lower frequencies.

After considering the limitations resulting from the frequency and line intensity dependence of $r_{\max }$ (cf. Eq. 64), the distribution function Eq. (61) can be integrated over frequency, and the result is given by

$$
\begin{aligned}
\Delta N(l) & \propto \operatorname{sign}(A) 10^{\frac{2 l t}{\sigma}} \times \\
& \times F\left(l, t, \sigma, i_{\mathrm{e}}, x_{\max }, \nu_{\max }, \gamma, l_{\min }, r_{\min }\right) \\
A & =\left(\frac{2 t}{\sigma}+1-\gamma\right) \\
l_{\min } & <l<l_{\max }=\frac{x_{\max }}{t}-r_{\min } .
\end{aligned}
$$

The functional behaviour of $F$ is given in Appendix E, $x_{\max }$ is the highest energetic level considered (acting as lower one for the lines accounted for) and $\nu_{\max }$ the maximum integration frequency chosen. In those cases where the level and line list are complete (or the complete ones are used), we have $x_{\max }=\nu_{\max }=i_{\mathrm{e}}$. Note, that the distribution actually depends on certain ratios, e.g., $t / \sigma, i_{\mathrm{e}} / \sigma$, $x_{\max } / \sigma$ and $\nu_{\max } / \sigma$ and not on the absolute numbers.

Summarizing the results concerning $F$ derived in Appendix E, we find that the frequency integrated distribution behaves rather similarly to the frequency dependent one. In total, three different slopes are possible, namely $2 t / \sigma, t / \sigma$ and $\gamma-1$, were the occurrence and position of the former two are controlled by the value of $\gamma$ being larger or smaller than a critical value $\gamma_{\text {crit }}=1+2 t / \sigma$.

Under typical conditions, however, the function consists of only two parts, namely a steeper, excitationdominated one with slope $2 t / \sigma$, and a second one with slope $\gamma-1$, similar to the frequency dependent distribution. The division is given at line intensity $l=x_{\max } / t-$ $\log g f_{\max }$, when we consider only those lines with a lower energy level below the cutoff energy $x_{\max }$ introduced above. Furthermore, in case of a frequency integration between 0 and $\nu_{\max }<i_{\mathrm{e}}$, the function preserves its shape for all $\nu_{\max }<i_{\mathrm{e}}-x_{\max }$, an effect which we have called the "saturation effect".

In principle, the constant of proportionality in Eq. (73) depends also on temperature and level distribution for the specified ion as well as on the exponent of the oscillatorstrength distribution. However, in the following we derive this constant from the requirement that the total line number is known (and to be found from the cumulative line number at $l_{\max }$, which is the maximum possible line intensity).

In order to check the validity and applicability of the above expressions, we have performed a number of test calculations in the same spirit in the previous section, 


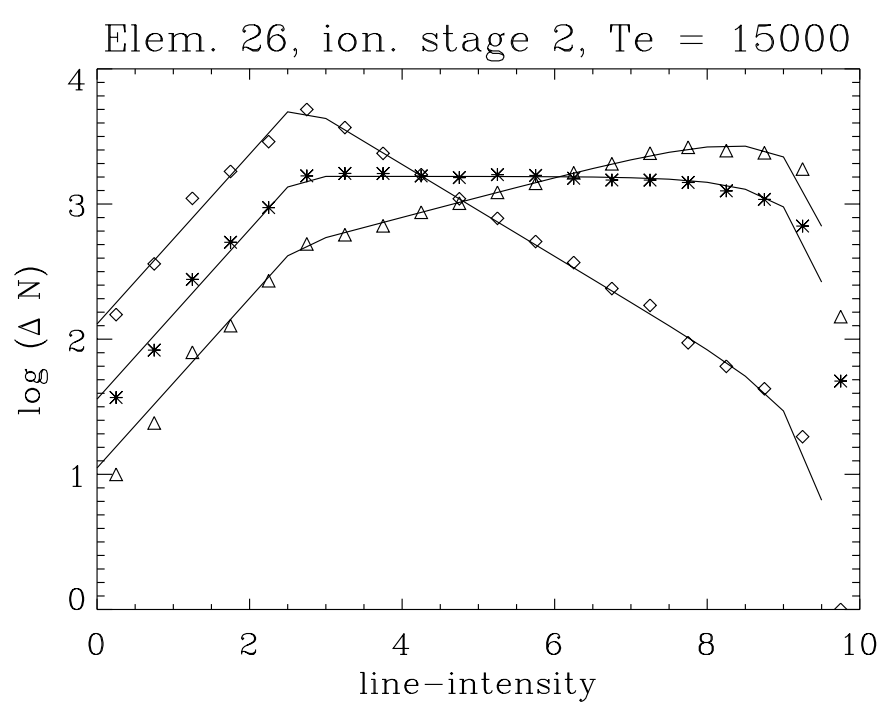

Fig. 12. Frequency integrated line intensity distribution functions of FeII at $T=15000 \mathrm{~K}$. Frequency range $0<x<$ $65 \mathrm{kK}$ (see text) and resolution 0.5 dex in intensity. Oscillator strengths simulated by Monte Carlo with $\gamma=1.0,0.66,1.15$ (asterisks, diamonds and triangles). Curves display the analytical result according to Eq. (73) with $i_{\mathrm{e}}=130 \mathrm{kK}, i_{\mathrm{e}} / \sigma=1.7$, $x_{\max }=63 \mathrm{kK}, l_{\min }=0$ and $r_{\min }=-7$, where the latter two quantities have been also used in the Monte Carlo simulations

i.e., for our atomic model of FeII and simulating the $g f$ distribution by a Monte Carlo process.

For our specific atomic model, we have $i_{\mathrm{e}} \approx 130 \mathrm{kK}$ (lines with larger energies resulting from levels ionizing to excited levels of FeIII were removed "by hand" in order to simplify the test), and, from our constraint of considering only those levels which under NLTE-conditions have large enough occupation numbers to form lines of significant strength (see Sect. 3.1 and next section), we have $x_{\max }=63 \mathrm{kK}$. For reasons of consistency, the integration was performed in the corresponding range $0<\nu<65 \mathrm{kK}$. From a first comparison, it turned out that $i_{\mathrm{e}} / \sigma=1.7$, which is also consistent with the average slope of the level distribution. The normalization constant was chosen in such a way that a total line number of $\log N=4.35$ was reached in the considered frequency range.

Since all other quantities defining the frequency integrated distribution functions are given as parameters of the Monte Carlo simulation $\left(\gamma, l_{\min }=0, r_{\min }=-7\right)$, a comparison of the analytical result and the simulated line intensity distribution should coincide for all temperatures and all values of $\gamma$, if the above expression were correct. An example is given in Fig. 12, and the agreement is obvious, note in particular the predicted steep slope for strong lines and the dependence of the weak lines' distribution on $\gamma^{7}$.

\footnotetext{
7 A likewise agreement has been found for simulations at $T=$ 5000 and $10000 \mathrm{~K}$.
}

Elem. 26, ion. stage 2, Te $=15000$

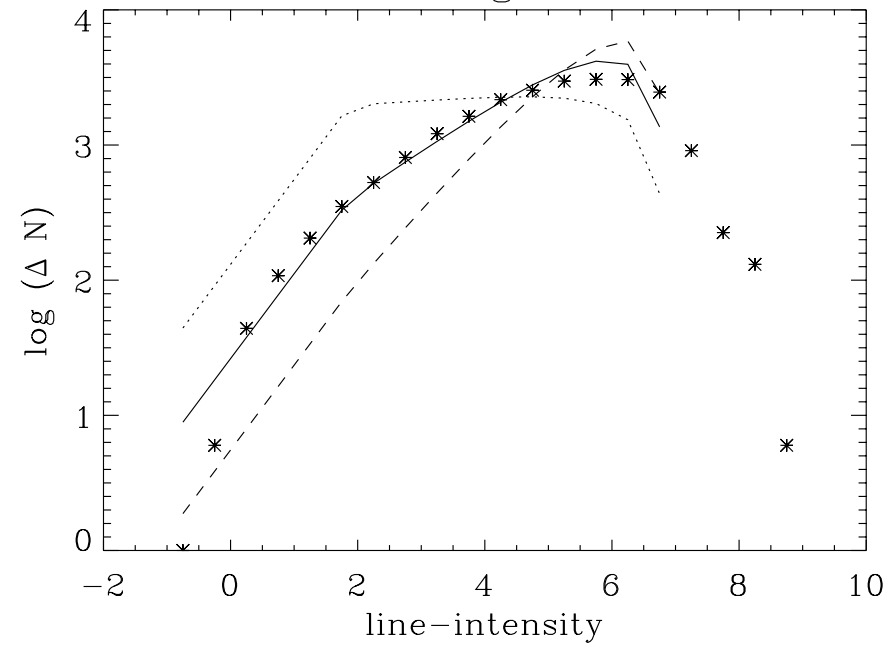

Fig. 13. As Fig. 12, using actual oscillator strengths (asterisks). Overplotted is the analytical result according to Eq. (73) with $i_{\mathrm{e}}, i_{\mathrm{e}} / \sigma$ and $x_{\max }$ as before; $l_{\min }=-0.75$ and $r_{\min }=-4.25$ (see text). Fully drawn: $\gamma=1.33$; dotted: $\gamma=1.03$; dashed: $\gamma=1.63$

In the next figure, we have investigated the most interesting question in this respect, namely how far the analytical description can deal with the real case. We have considered the same situation as above, however used the actual oscillator strengths. Thus, by this comparison we can check our hypothesis whether the frequency integrated distribution is less influenced by curvature effects than the distribution defined at specific frequencies, and ask for the effective $\gamma$-exponent of the underlying oscillator strength distribution.

Compared to the last simulations, we have changed only the parameters $l_{\min }$ and $r_{\min }$ in the analytical expression, and tried to fit the actual distribution (asterisks) by varying the value of $\gamma$. The choice of the former quantities relates to the oscillator strength distribution function of FeII (Fig. 11, triangles), with a maximum $\log g f$-value $\left(=-l_{\min }\right)$ of 0.75 and the distribution becoming incomplete at $r_{\min }=-4.25$. Figure 13 gives the results for three different values of $\gamma$.

Two points are worth noticing. First, the actual distribution can be fitted extremely well by our analytical approach (the same degree of precision was reached at different temperatures), and the effective $\gamma$ is of the order 1.3, i.e., again representing the "canonical" value. This is not too surprising since a major part of the distribution actually follows this slope (Fig. 11). Second, a comparison with the other simulations with different $\gamma$ illuminates the role of this quantity: Although (and in agreement with our findings for the frequency dependent line statistics) the slope of the distribution for strong lines remains almost unaffected by $\gamma$ and is much more influenced by temperature via excitation, the width of the distribution and its vertical offset depend strongly on this quantity! 
This behaviour is readily understood, if one remembers the fact that $\gamma$ primarily controls the distribution of weak lines: for $\gamma=1$, the number of these lines remains constant (until $l_{\max }$ ), whereas for $\gamma<1$ it decreases with increasing $l$ and vice versa for $\gamma>1$. This is not only true for frequency dependent distribution functions but even more for integrated ones, since the threshold line intensity $l_{\mathrm{T}}$ decreases with increasing frequency. The presence of this effect is particularly demonstrated by our simulations for the high temperature case shown here for this reason.

An additional constraint for the resulting distribution is the conservation of total line number: If $\gamma<1$ and the number of lines is small for large $l$, this has to be compensated for by a larger number of lines at low $l$. In contrast, for larger $\gamma$ and a consequently increasing number of weak lines the number of strong lines has to be smaller. Finally, also the width of the distribution at maximum $\Delta N$ is affected, since this region becomes flat for $\gamma=1$, and the according width displays a maximum.

For reasons of brevity, we have presented here only the case of FeII, due to its importance for radiative driving and its large number of lines leading to a low degree of statistical noise. Of course, we have checked our approach also for different ions (e.g., belonging to the CNO-group), and found a satisfactory agreement in any case.

Thus, the frequency integrated line intensity distribution of various ions can be described analytically assuming a power-law distribution for the $g f$-values, and we have illuminated the role of the corresponding exponent above.

The results presented here may turn out to be useful also in another regard. Since the dependency of the frequency integrated line-intensity distribution on the various parameters is understood, the procedure can be inverted to check for the completeness of atomic data bases. This check can be easily performed, since the calculation of the distribution function (per ion) is simple, involving only Boltzmann excitation. From an analysis of the turn-over points and the width of the distribution (as a function of input temperature), it is thus possible to derive, e.g., the effective values of $x_{\max }$ and $g f_{\min }$, and data gaps will show up immediately. By a variation of $\nu_{\max }$, it will be also possible to constrain the completeness as a function of frequency, at least in those cases where the aforementioned "saturation effect" does not play a role.

\subsubsection{From line intensity to line-strength distribution functions}

The next important step concerns the transition from line intensities to line-strengths, where we have the following relation (cf. Eq. (60) vs. 6):

$$
10^{-l}=g f\left(\frac{n_{l} / g_{l}}{n_{1} / g_{1}}\right)^{*}
$$

$$
\begin{aligned}
\frac{k_{\mathrm{L}}}{10^{-l}} & \approx b_{l} \frac{\pi e^{2}}{m_{\mathrm{e}} c} \frac{n_{1}}{g_{1}} \frac{\lambda}{\sigma_{e} v_{\mathrm{th}}}= \\
& =\left(b_{l} X_{j k} \epsilon_{k}\right) \frac{\frac{\pi e^{2}}{m_{\mathrm{e}} c} \lambda}{\left(1+I_{\mathrm{He}} Y\right) \sigma_{\mathrm{T}} g_{1} v_{\mathrm{th}}}
\end{aligned}
$$

The asterisk denotes the LTE-value with respect to the ion's ground-state " 1 ", $b_{l}$ is the NLTE departure coefficient of the lower level of the considered transition - again defined with respect to the ground-state (cf. Sect. 3.1.2), $X_{j k}$ the ionization fraction of ion " $j$ " with abundance (relative to hydrogen) $\epsilon_{k}$ of element " $k$ ". $I_{\mathrm{He}}$ is the number of free electrons per Helium atom, $Y$ the Helium abundance, $g_{1}$ the statistical weight of the ion's ground-state and $\sigma_{\mathrm{T}}$ the cross-section of electron scattering. Note, that the thermal speed $v_{\text {th }}$ refers to the nominal value for hydrogen (cf. Sect. 2.2). In the above equation, we have neglected the contribution of stimulated emission.

Inserting typical parameters, this relation can be expressed as

$$
\begin{aligned}
& \log k_{\mathrm{L}} \approx-l+6+\log S_{l j k} \\
& S_{l j k}=\left(b_{l} X_{j k} \frac{\epsilon_{k}}{\epsilon_{\mathrm{Fe} \odot}}\right) \frac{\left[\frac{\lambda}{1000 \AA}\right]}{\left[\frac{1+I_{\mathrm{He} Y} Y}{1.2}\right]\left[\frac{g_{1}}{4}\right]\left[\frac{v_{\mathrm{th}}}{27 \mathrm{~km} \mathrm{~s}^{-1}}\right]},
\end{aligned}
$$

i.e., $\log k_{\mathrm{L}} \approx-l+6$ for iron ions in hot star winds if the ionization fraction were unity and NLTE-effects were small. From the definition of $S_{l j k}$ it is obvious, that - per ion the only quantities which vary as a function of considered transition are $b_{l}$ and $\lambda$. Thus, it should be possible to relate the line-strength distribution function of a given ion to the corresponding line intensity distribution function, since the (effectively) contributing range in wavelength (factor $\lambda$ ) is not too large (this even more, if we consider flux-weighed quantities) and the variation of the departure coefficients is moderate as well, if we recall the scaling relations of important NLTE levels (Sect. 3.1.2). Thus, the variation concerns only those excited levels which are connected to a ground or meta-stable level and is proportional to the dilution factor of the chosen reference point (or a steeper function of $r$, if optical depth effects of lines were to be included). These levels, however, play a role almost only in light ions, whereas all decisive lines of heavy ions are connected directly to meta-stable levels (and the ground state).

Before we can relate the line intensity to the linestrength distribution function, we have to account for a parameter which is essential concerning this objective. Whereas for the (complete) distribution of line intensities all levels up to the ionization edge are significant (excitation by Boltzmann law), the line-strength distribution accounts for a lower number of levels and lines, namely those which are actually occupied under NLTE conditions. These are mainly those lines with a lower level attributed to one of the three categories defined in Sect. 3.1.2.

In other words, NLTE effects introduce an effective cutoff for contributing (lower) levels, $x_{\max }<i_{\mathrm{e}}$, already 
introduced in Eq. (73). As discussed in Appendix E, this quantity (instead of the ionization potential in the LTEcase) now controls the transition value between the excitation vs. oscillator strength dominated part of the distribution function, i.e., this point is shifted significantly towards higher line-strengths. In consequence, the apparent distribution resembles that of an ion with low-lying ionisation potential! Of course, only part of this effect becomes visible in the following, since the level lists in our data base have been designed a priori to be complete only up to essentially occupied levels.

An example for the correspondence of line-strengths vs. line intensities is given in Fig. 14. The asterisks display the NLTE (Sect. 3.1) line-strength distribution for FeIv, and the fully drawn curve shows the corresponding analytical line intensity distribution, however plotted as a function of $-l-0.1$, with cutoff energy at $x_{\max }=170 \mathrm{kK}$ (for details, see caption). Note, that the highest level in our data base lies at $210 \mathrm{kK}$, and $i_{\mathrm{e}} \approx 440 \mathrm{kK}$.

From the perfect agreement, it is evident that the linestrength distribution can be actually described in analogy to our previous results for line intensities, where in view of Eq. (76) - the "average" shift is given by $\log S=-6.1$, and this shift originates mainly from the rather low ionization fraction of FeIV at $40000 \mathrm{~K}$, of order $10^{-5}$. In this example, the distribution is not "saturated", i.e., $\nu_{\max }>i_{\mathrm{e}}-x_{\max }$, which should lead to two distinctive slopes in the first part of the distribution, namely $2 t / \sigma$ and $t / \sigma$ (Appendix E). Even this subtle effect is visible in the actual distribution!

\subsubsection{Summation over all contributing ions}

As we have understood now, the distribution function per ion consists of a steep and a flatter part, were the transition is controlled by the ratio $x_{\max } / t$. The major problem left is the summation over all contributing ions, since, of course, each ion has its own specific $S_{j k}$ value. Thus, even if the line intensities were similarly distributed for each ion, the transformation (= horizontal shift) to the linestrength space might produce unpredictable results if all ions are considered in parallel, as required, e.g., for the calculation of the line-force.

To facilitate the investigations, we have performed some test calculations before considering the real case, again by using the actual atomic models and NLTE occupation numbers (ionization and excitation have been calculated according to Sect. 3.1), however simulating the oscillator strength distribution via Monte Carlo.

Moreover, at first we have concentrated on ions with should behave rather similarly due to their electronic structure (here: $\mathrm{Ti}$ to $\mathrm{Cu}$, in the following "iron group elements", and later the "light ions", C to Ca) and assumed an equal abundance in order to distinguish between ionization and abundance effects. The chosen abundance

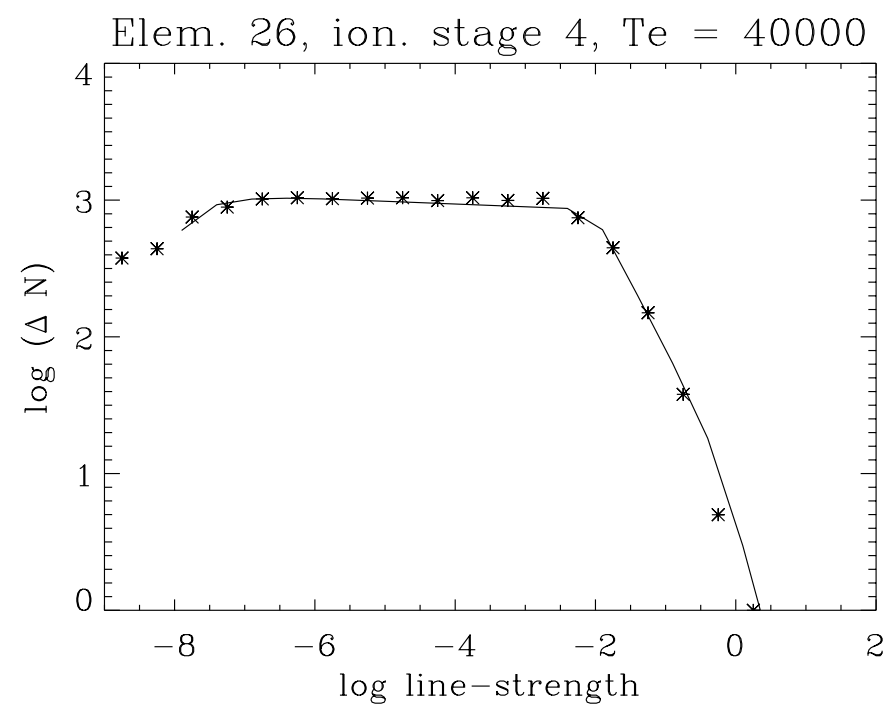

Fig. 14. Asterisks: (Frequency integrated) Line-strength distribution function for FeIv, $T_{\text {eff }}=40000 \mathrm{~K}$, dilution factor $W=0.5, n_{\mathrm{e}} / W=10^{12}$ and resolution 0.5 dex in line-strength. Fully drawn: Analytical line intensity distribution function, plotted over $-l-0.1$ (i.e., $\log S \approx-6.1$ ). Parameters (except $x_{\max }$, which results from the calculated distribution) consistent with our data base: $\gamma=1.0, i_{\mathrm{e}} / \sigma=6.5, i_{\mathrm{e}}=440 \mathrm{kK}$, $\nu_{\max }=400 \mathrm{kK}, x_{\max }=170 \mathrm{kK}, l_{\min }=-0.7, r_{\min }=-5.5$ and $\log N\left(l_{\max }\right)=4.1$

resembles the maximum solar value for elements of the iron group, namely for Fe itself $(\log \epsilon=-4.5)$.

Figure 15 (asterisks) gives the resulting frequency integrated line-strength distribution function for those iron group elements and a simulated $g f$-distribution with $\gamma=1, r_{\max }=0$ and $r_{\min }=-7$, for the same atmospheric conditions as in Fig. 14. Obviously, three different groups are present, which can be easily disentangled due to our knowledge of $\gamma$ (input for Monte Carlo simulation).

This is done in Fig. 16, by means of our analytical description (Eq. 73) and at first in line intensity space. Note, that the displayed solution is only one of a number of other possibilities: For low-lying values of $x_{\max }$, which have been derived from the onset of the flat $\gamma=1$ distribution and result from the effective "NLTE cutoff" (here: $\approx 140 \mathrm{kK}$ ) and as long as the function is "saturated", which is the case in our example, the actual value of the ionization energy is unimportant (cf. Eq. E8). Decisive is only the parameter $\sigma$, controlling the steeper part of the distribution function via $(2) t / \sigma$.

After summing up the three different components and plotting them as a function of $-l+6$, the line-strength distribution found in Fig. 15 (asterisks) can be easily simulated and is displayed by the bold line in this figure. Thus, although the actual function consists of a variety of contributing ions (at least 20 important stages in the considered case), only a small number of clearly different groups behaving similarly amongst each other is finally present. The first group consists of dominant ionization 


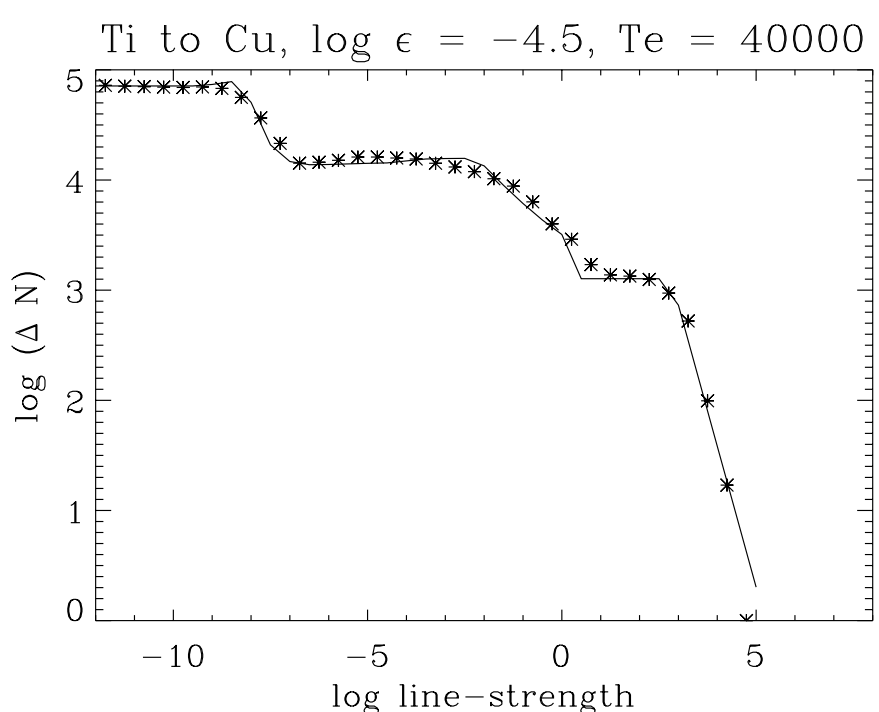

Fig. 15. Asterisks: Line-strength distribution function for the elements $\mathrm{Ti}$ to $\mathrm{Cu}$ (all ionization stages), with equal abundances $\log \epsilon=-4.5$. Oscillator strength distribution simulated by Monte-Carlo with $\gamma=1$. Fully drawn: Analytical result of line intensity distribution (summing up the three components of Fig. 16), plotted as function of $-l+6$

stages $(\log S \approx-1)$, the second one of minor stages comparable to FeIV in Fig. $14(\log S \approx-6)$, and the third one comprises the weakest lines from ions with negligible populations $(\log S \approx-12)$. Before commenting on this similarity in behaviour, let us firstly demonstrate that our findings are not only by chance.

Figure 17 shows the same situation as displayed in Fig. 15 (asterisks), with the only change of $\gamma$ from 1 to 0.8. Actually, even without simulating the new function, it is clear that our argumentation still holds. Again, we can distinguish three groups, and, consistent with our earlier findings, the plateaus from Fig. 15 related to $\gamma=1$ have changed into declining slopes related to the new slope of $\gamma=0.8$. If we now apply the identical parameters as in Fig. 16 (accounting, of course, for $\gamma=0.8$ ) and overplot the summed result, we find again a satisfactory agreement. This clearly indicates that the combination of parameters chosen for the individual components are of the correct order, and that the line-strength distribution function actually consists of three different components, with similar effective cutoff energies.

Figure 18 (asterisks) displays now the actual case, i.e., without any manipulation of the oscillator strengths. A comparison with the $\gamma=1.0$ simulation (Fig. 15) shows that the differences are only small: Again, three distinct distributions are visible, where the transition from group one to two (roughly at $\log k_{\mathrm{L}} \approx 0$ ) is no longer as pronounced as before. This might indicate that the actual $g f$-distribution for the lines of this group is steeper than $\gamma=1.0$ The second group, however, is consistent with $\gamma=$ 1.0 , as is obvious from the plateau at $\log k_{\mathrm{L}}=-3 \ldots-8$.

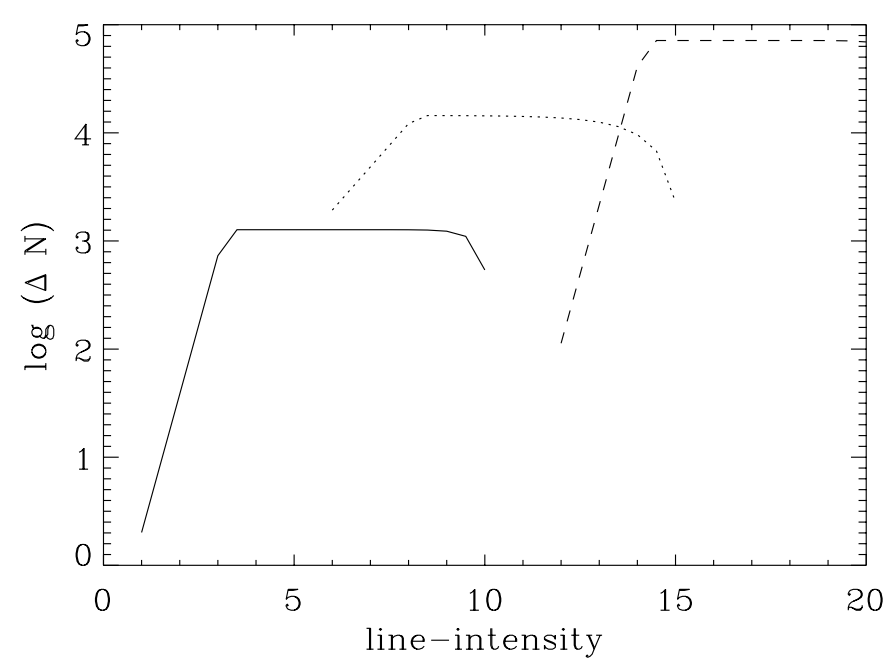

Fig. 16. Analytic line intensity distribution functions for the three components visible in Fig. 15. Common parameters: $\gamma=1.0, i_{\mathrm{e}}=800 \mathrm{kK}, \nu_{\max }=400 \mathrm{kK}, x_{\max }=140 \mathrm{kK}, l_{\min }=$ $0, r_{\min }=-7$. Note that the actual value of $i_{\mathrm{e}}$ is unimportant as long as $\nu_{\max }<i_{\mathrm{e}}-x_{\max }$ (see text). Individual parameters: strong lines (fully drawn): $i_{\mathrm{e}} / \sigma=8, \log N\left(l_{\max }\right)=4.25$, "ionization shift" (see Eq. (76) and text) $\log S=-1$; weak lines (dotted): $i_{\mathrm{e}} / \sigma=2.5, \log N\left(l_{\max }\right)=5.3, \log S=-6$; very weak lines $($ dashed $): i_{\mathrm{e}} / \sigma=8, \log N\left(l_{\max }\right)=6.0, \log S=-12$

The details of this figure give an the answer to the question raised above, namely, why the sum of rather complex individual distributions (for the dominant iron group ions at $T_{\text {eff }}=40000 \mathrm{~K}$, see caption) can be described in such simple terms as above.

From the last panel, it is obvious that ionization stages $\mathrm{V}$ and VI represent the first group, and stage IV is identical with the second one. Additionally, from the sharp decline of the bold line (sum of stages IV, V, VI) at the end of the plateau, where the complete distribution function including all ions is rising again, it is clear that the third group (not analyzed here) consists of "real" trace ions (mostly stage III).

The upper three panels show the distribution of the individual species amongst the various ionization stages (as well as the appropriate sums). Neglecting certain subtleties, all elements of a given ionization stage behave similarly. Thus, we can speak of line-strength distribution functions of specific ionization stages instead of individual ions (equal abundances provided).

The origin of this similarity bases on the only minor differences (in a statistical sense) in atomic structure of the iron group elements under consideration, especially with respect to ionization rates (giving rise to similar ionization fractions), a rather low lying effective cutoff energy and a level density parameter $\sigma$ which is small enough to induce the steep increase in the first part of the distribution. Note already here that the according slope (the steeper one!) is roughly equal for all kinds of ions and consequently also for the summed distributions, independent 


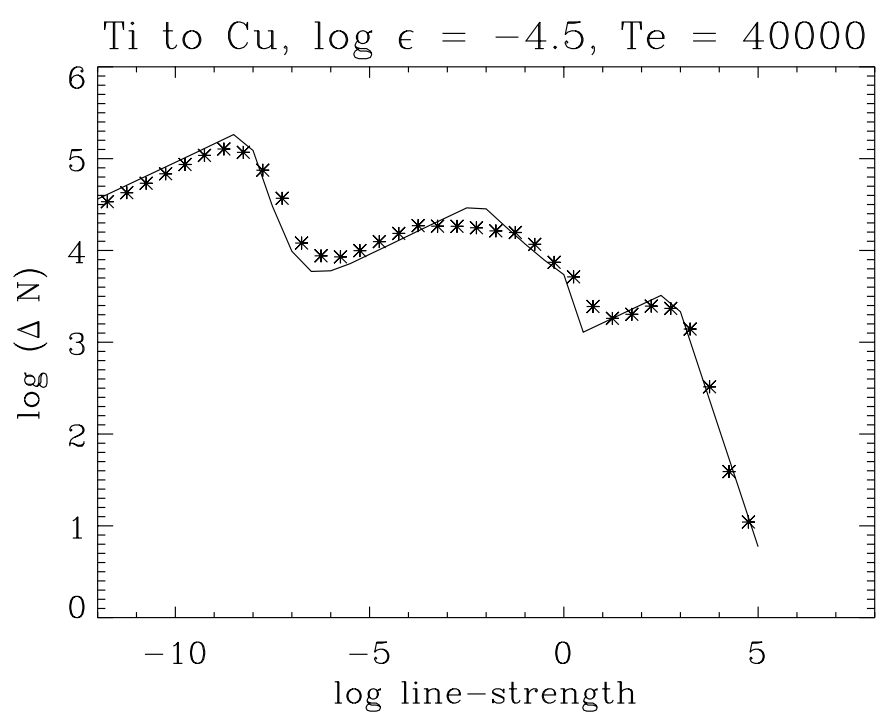

Fig. 17. As Fig. 15, however with exponent $\gamma=0.8$ and corresponding line intensity distributions, plotted as functions of $-l+6$. Parameters of individual line intensity distributions as in Fig. 16

of temperature (provided, of course, the abundances were equal). This equality is clearly shown in Figs. 18, 19 and 20 and translates to a similarity in $2 t / \sigma$, being of order unity. Since the value of $2 t$ varies from 32 to 160 in the appropriate units (corresponding to $10000 \ldots 50000 \mathrm{~K}$ ), this indicates that the (effective) level-density parameter $\sigma$ (the smaller, the steeper is the level-distribution as function of energy) has to vary in concert with these numbers. With respect to the cases discussed already as well as from the argument that an increase in ionization stage/potential inevitably leads to an increase of $\sigma$ (fewer levels distributed over a larger energy interval), this behaviour is not surprising at all.

In addition to this similarity in excitation dominated slope, the $g f$-distributions are similar as well, with $\gamma$ between $1 \ldots 1.3\left(<\gamma_{\text {crit }} \approx 2\right)$, dominating the individual distributions after the first two or three decades of steep incline.

In consequence, the total line-strength distribution has a "staircase"-like structure, where each staircase corresponds to a specific stage and is controlled by the sequence excitation/oscillator strength distribution. The horizontal width of these staircases depends mostly on the prevalent ionization fraction.

\subsubsection{Temperature dependence}

Since "only" the first seven to ten decades of line-strength are important for line-driving, only those trace ions do contribute to the total distribution which have a significant ionization fraction (and abundance, cf. Sect. 4.2.6) not below roughly $10^{-4} \ldots 10^{-5}$ as well as a larger number of lines than the dominant ionization species. In the

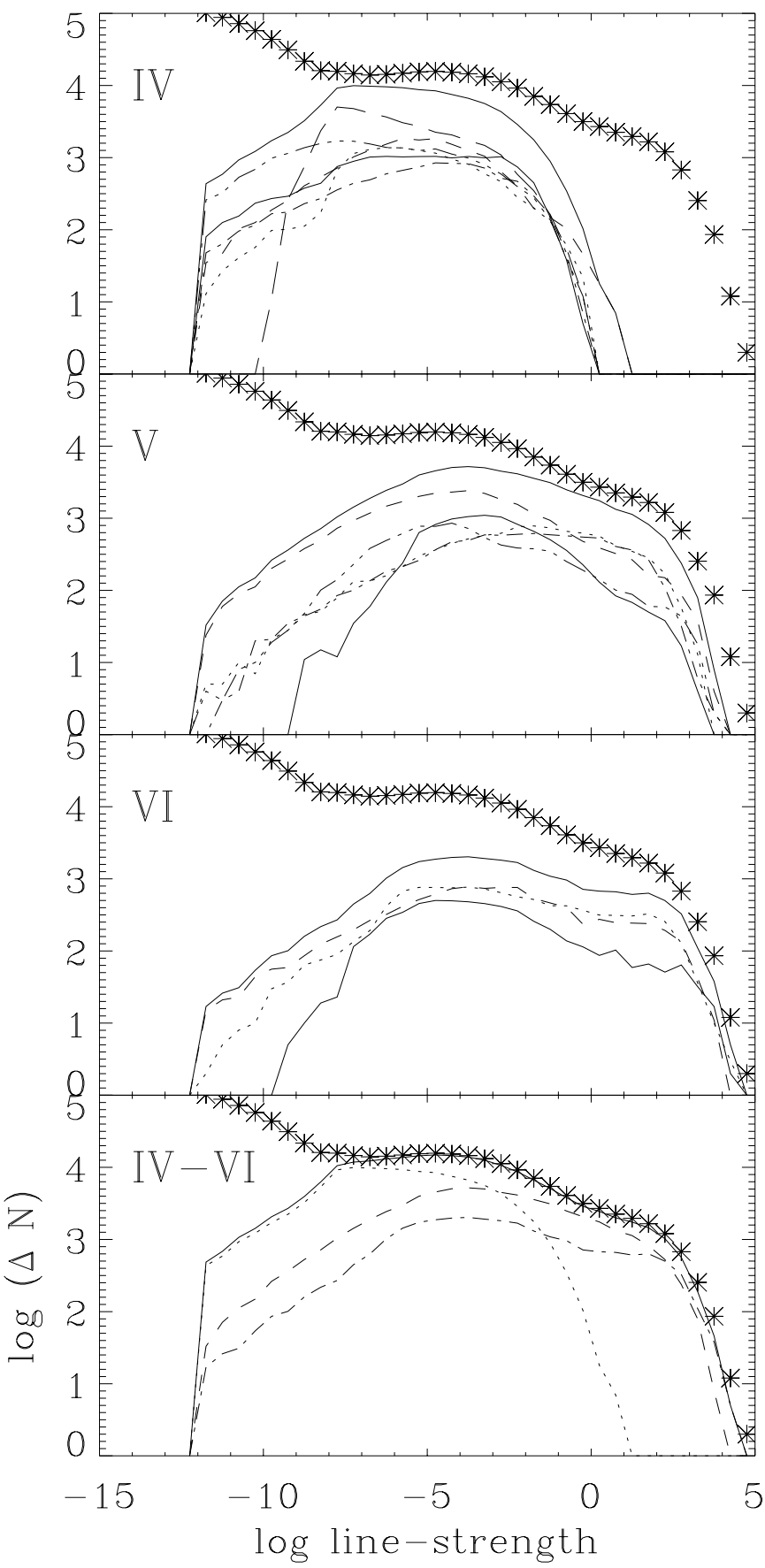

Fig. 18. Asterisks: Actual line-strength distribution function for the elements $\mathrm{Ti}$ to $\mathrm{Cu}$ (all ionization stages), with equal abundances $\log \epsilon=-4.5$, compared to the most important individual components. Atmospheric parameters as in Fig. 14. Lower panel: contribution by most important ions of stage IV (dotted), V (dashed) and VI (dashed-dotted), as well as the sum of these three components (bold line). Upper panel: most important individual components of ionization stage IV. Fe (fully drawn), $\mathrm{Ni}$ (dotted), $\mathrm{Cu}$ (dashed), Co (dashed-dotted), $\mathrm{Mn}$ (dashed-dotted-dotted and Cr (long-dashed), as well as the sum of these ions (bold). Second panel: same as above, however for ionization stage $\mathrm{V}$ and elements $\mathrm{Fe}, \mathrm{Ni}, \mathrm{Cu}, \mathrm{Co}$, Mn and sum of them. Third panel: ionization stage VI for Fe, $\mathrm{Ni}, \mathrm{Cu}$ and sum 
other case, i.e., if the line number is smaller, these trace ions are barely visible in the summed distribution: Then, the $\gamma-1$ power-law increase of lines from major species (which extends $r_{\min } \approx 5 \ldots 7$ decades in line-strength from the turnover point to the "left") dominates the essential part of the distribution.

Especially for iron group elements, the (total) line number per ion increases strongly with decreasing ionization stage due to the increasing complexity of electronic structure. Thus, at higher temperatures a significant contribution from trace ions of lower stages is actually possible, since these have the required larger line number. At the lower temperature end of radiatively driven winds (roughly $T_{\text {eff }} \approx 8000 \mathrm{~K}$ ), however, all (important) trace ions have necessarily a higher degree of ionization than the major ones and consequently do not (or only marginal) contribute to radiative driving.

This effect is clearly visible in Fig. 19, where we have plotted the line-strength distribution for iron group elements (again using equal abundances) as function of temperature. At the lowest temperature displayed $\left(T_{\text {eff }}=\right.$ $10000 \mathrm{~K}$, bold line), the enormous line number from ionization stages II and III dominates the first twelve decades. Trace ions (below $\log k_{\mathrm{L}}=-5$ ) have too few lines (as well as negligible ionization fractions) to be of any importance. At $20000 \mathrm{~K}$ (dotted), the situation is slightly different. Here, stages III and IV are essential, however a 2nd peak shows up indicating the presence of stages II. This trend continues to higher temperatures, e.g., for the case discussed above $\left(T_{\text {eff }}=40000 \mathrm{~K}\right.$, asterisks), two kinds of trace ions become visible, namely stage IV in the middle part and stage III with even more lines at weakest linestrengths. At the highest temperature $\left(T_{\text {eff }}=50000 \mathrm{~K}\right.$, dashed-dotted), the dominant species are vI and partly VII. Ions from stage V contribute significantly, whereas stage IV with its typical $\gamma=1$ distribution is visible only at weakest line-strengths. Thus and in total, we see a clear dominance of one or two major ionization species in the complete temperature regime.

Let us now concentrate on the decisive part of the distribution (down to, say, $\log k_{\mathrm{L}}=-2$ ). With decreasing temperature, the maximum line-strength $k_{\max }$ increases, which is primarily related to the presence of low-lying meta-stable levels acting as quasi ground states, which are missing in the higher ionization stages. Most important, however, is the difference in total line-number! Whereas at the hottest temperatures the transition point occurs at a line number $\log \Delta N \approx 3$, at lower temperatures a factor of 10 more lines are present at this point. This difference, of course, bases on the increasing number of lines with decreasing ionization stage $\Delta N \propto a^{2} \sigma \sim 1 / \sigma \sim 2 t$ (Eq. (61), Allen (1966), his Eq. (3.6) and accounting for $2 t / \sigma \approx 1$ ). Note, however, that the position of the transition point itself $\left(x_{\max } !\right)$ and both slopes (before and after) remain essentially unaffected.

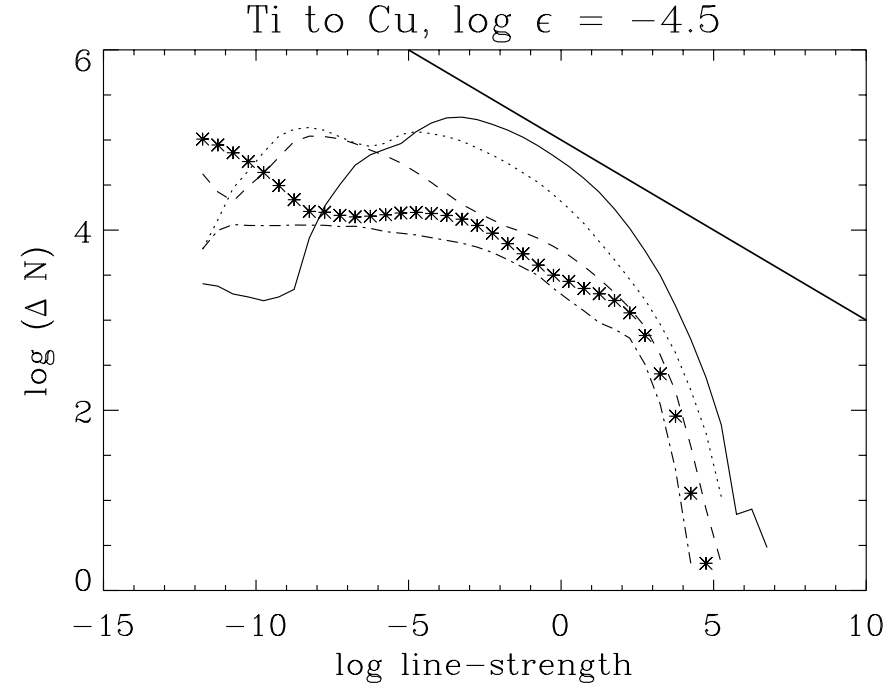

Fig. 19. Line-strength distribution function for the elements Ti to $\mathrm{Cu}$, with equal abundances $\log \epsilon=-4.5$, as a function of temperature (remaining atmospheric parameters as in Fig. 14). $T_{\text {eff }}=10000 \mathrm{~K}$ (fully drawn), $20000 \mathrm{~K}$ (dotted), $30000 \mathrm{~K}$ (dashed), $40000 \mathrm{~K}$ (asterisks, cf. Fig. 18) and $50000 \mathrm{~K}$ (dasheddotted). For comparison, the straight line shows a power-law distribution with slope corresponding to $\gamma=1.2$

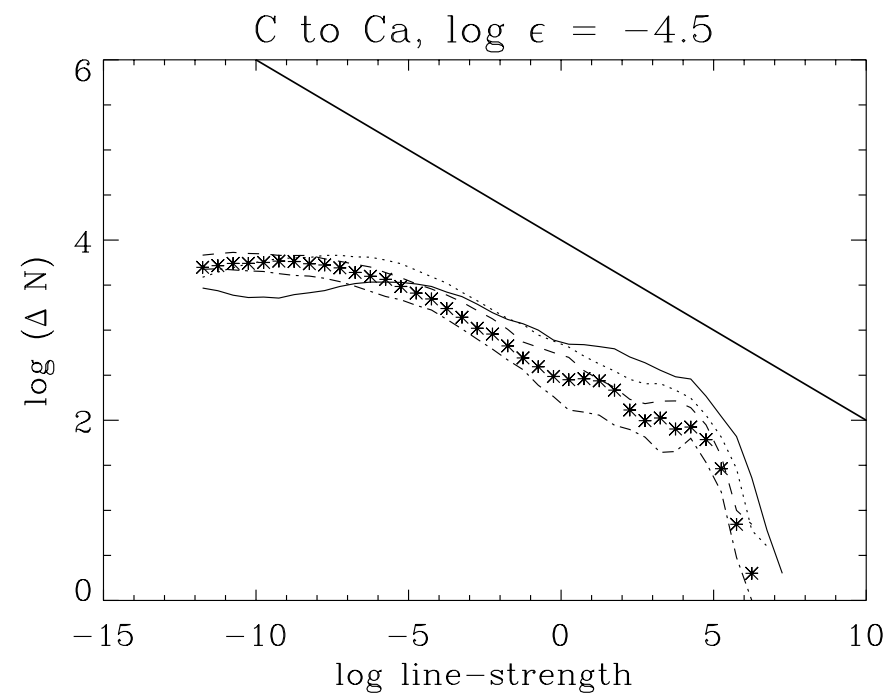

Fig. 20. As Fig. 19, however for the light ions $\mathrm{C}$ to $\mathrm{Ca}$ and equal abundances $\log \epsilon=-4.5$

So far, we have concentrated on iron group elements. As we will see soon, light ions play an equally important role, although the total number of lines from those elements is significantly smaller. Figure 20 shows the corresponding line-strength distribution, again with equal abundances and as function of temperature. In contrast to above, the maximum value of $k_{\mathrm{L}}$ remains rather constant, since the strongest lines are formed by resonance transitions, so that excitation effects are unimportant for the definition of $k_{\max }$. The largest differences occur at intermediate line-strengths. They are connected to the large 

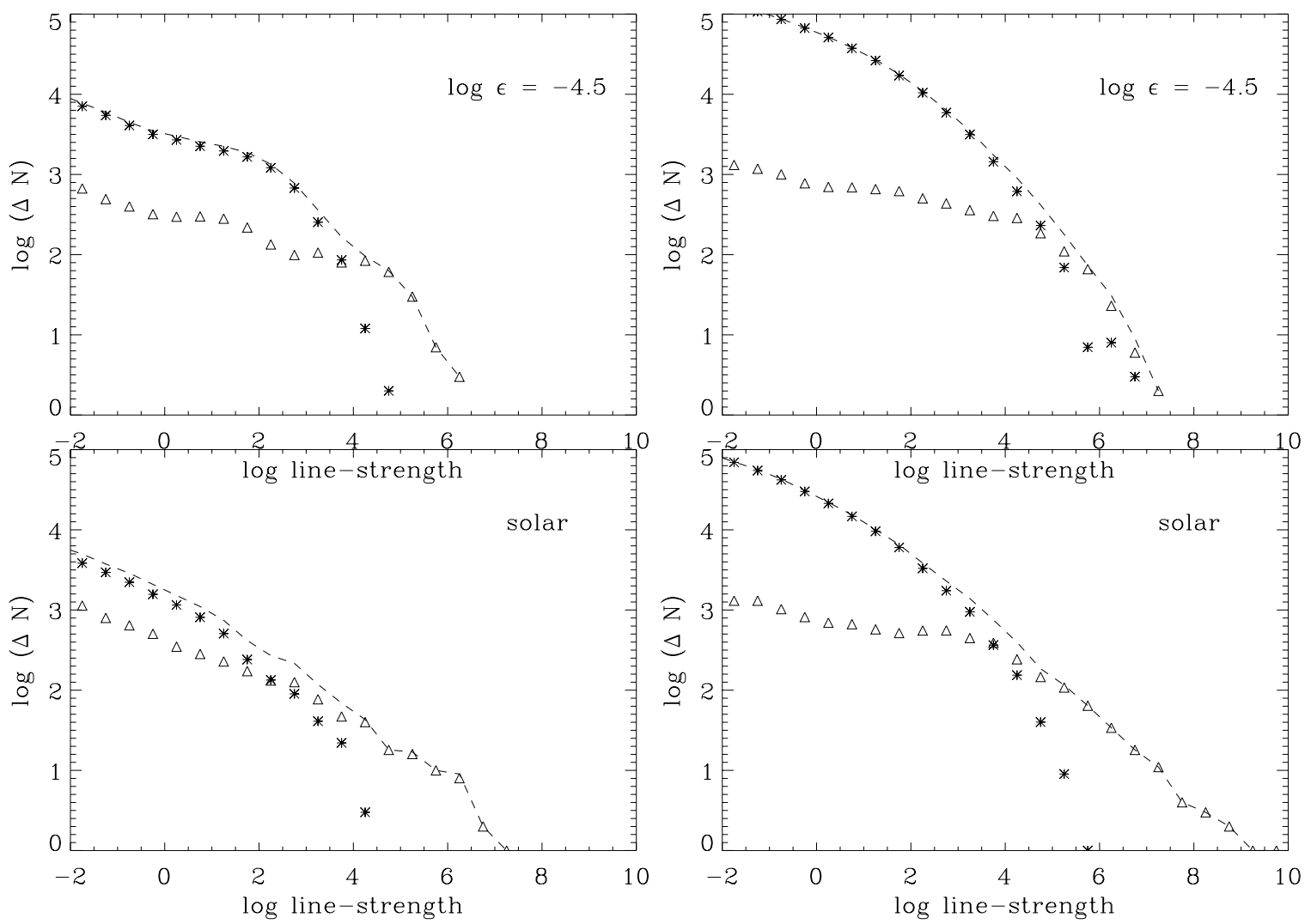

Fig. 21. Summing up the line-strength distribution functions for iron group elements and light ions. Atmospheric parameters as in Fig. 14, for a temperature of $40000 \mathrm{~K}$ (left) and $10000 \mathrm{~K}$ (right panel). Upper row: Distribution functions for iron group elements (asterisks) and light ions, (triangles), assuming equal abundances $\log \epsilon=-4.5$. The dashed line gives the total linestrength distribution function. $\alpha(40000 \mathrm{~K})=0.41, \alpha(10000 \mathrm{~K})=0.34$, derived fron least square fit to cumulative distribution in decisive $k_{\mathrm{L}}$-range. Lower row: As above, for solar abundances and including $\mathrm{H}$, He. $\alpha(40000 \mathrm{~K})=0.57, \alpha(10000 \mathrm{~K})=0.48$

number of resonance lines from lower ionization stages in the region around $600 \AA$ (convergence to ionization edges), whereas the corresponding lines of the higher stages are situated well below our frequential cutoff at $250 \AA$. On the whole, however, the distribution functions are much more similar when the temperature is varied, compared to the iron group case, and the overall line number is smaller everywhere. The reason for this difference is readily understood, if we account for the vanishing number of metastable levels in light ions, so that the group of lines with a meta-stable level as lower one (which comprises the majority of lines for iron group elements) is completely missing. Again, the excitation dominated part shows (virtually) no reaction on temperature, i.e., $2 t / \sigma \approx 1$ as discussed above.

4.2.6. Total line-strength distribution functions and the influence of relative abundances

In Fig. 21, we have added now the contribution of light and heavy ions, both for the case of a hot wind at $40000 \mathrm{~K}$ as well as for a rather "cool" wind at $10000 \mathrm{~K}$. Additionally, we study the influence of accounting for realistic abundances, e.g., a solar mixture. Most importantly, by giv- ing up the uniform composition, the distribution function of heavy elements is shifted to the left (to lower linestrengths), since all contributing elements behave rather similarly (Fig. 18), however the (solar) abundance of elements different from $\mathrm{Fe}$ is smaller than the value $\log \epsilon=$ -4.5 adopted so far. Accordingly, the light ions' distribution function is shifted (to a lesser extent) to the right ( $\epsilon$ larger than for Fe on the average).

Thus, the difference between maximum line-strengths is increased if a solar composition is accounted for. The effect seems to be especially large for the cooler wind, where $k_{\text {max }}$ (light ions) is increased by 2 dex: At these temperatures, the Hydrogen Lyman lines (and, to a lesser extent, the HeII Lyman lines), which are insignificant at hotter temperatures, show up at largest line-strengths, both because of the larger ionization fraction of neutral hydrogen (and HeII) as well as their much higher abundance, compared to the metals.

Additionally, the resulting distribution functions (solar case) show even less structure than in the case of uniform abundance, simply because there is a larger scatter of the product $\left(X_{j k} \epsilon_{k}\right)$ (Eq. 75$)$, which leads to a larger variation of $S_{l j k}$ (Eq. 76) and consequently to a smoothing of 
any inherent ("staircase") structure which is still visible in the case of uniform abundances (e.g., Fig. 20).

Accounting now for these differences as a function of abundance as well as the intrinsic differences in the line statistics of iron group elements vs. light ions discussed previously, it becomes evident what controls the slope of the total line-strength distribution.

At first and for large line-strengths, the distribution is dominated by the behaviour of light ions, and it is the steeper, excitation influenced part of their distribution which plays the important role. Since a variety of abundances is present, the distribution is smoother and wider (more, however less pronounced staircases!). Thus, the according slope is flatter than the value of unity found in the case of equal abundances. Since the local slope translates to $\alpha=1-s$ with $s=\left|\operatorname{dog} \Delta N / \operatorname{dlog} k_{\mathrm{L}}\right|, \alpha$ becomes larger than zero in this domain (see also Fig. 25). For a cool plasma, the influence of the $\gamma=4 / 3$ distribution of $\mathrm{H}$ (Sect. 4.1) flattens the curve additionally.

On the other hand, the left part of the distribution (low $k_{\mathrm{L}}$ ) is controlled by iron group elements, due to their much larger line number. To obtain a situation where the light ions were of any influence in this range would require a mixture with a very small abundance of heavy elements, compared to the CNO group (Pop. III stars?).

The specific influence of the solar composition (actually, only the ratio and not the absolute numbers is relevant!) is evident from a comparison of both rows in Fig. 21 and the corresponding dashed lines, giving the total distribution functions. As discussed above, this abundance ratio introduces a larger separation of the two components. If the abundances were equal (upper row), the transition region between strong and weak line-strengths controlled by the light and heavy ions, respectively, is rather small. Thus, below the cutoff (effective $x_{\max }$ !) of the light ions, the distribution is suddenly dominated by the distribution of the heavy elements with their much larger line number, and the steep slope (order unity) of the first part of the total distribution function is continued, until finally the $g f$-dominated part becomes visible.

For solar abundance ratios (lower row), the intermediate range is much wider, and, accordingly, the transition to the flatter, $g f$-dominated part from iron group elements occurs in a rather smooth way. In connection with the fact that for a mixture of abundances the first part is flatter anyway, we find $\alpha>0$ for almost all $k_{\mathrm{L}}$, since $\alpha=1-s$ with $s<1$ in the first part, $\alpha \approx 2-\gamma$ with $\gamma=1 \ldots 1.3$ for the lowest contributing line-strengths and has values in between at intermediate strengths.

Again: If there were no difference in the abundance of light and heavy ions, the total distribution function would be steeper (significantly smaller $\alpha$ ) and much more curved compared to the solar case.
From Fig. 21 it becomes also clear why the derived $\hat{\alpha}$ values decrease for decreasing temperature (cf. Table 2$)^{8}$. At lower temperatures (right panel), there are simply more iron group lines present (esp. FeII, III, IV), compared to the rather constant line number of light ions. Thus, by lowering the temperature, the line-distribution becomes progressively steeper, especially at intermediate line-strengths, which reduces the corresponding $\alpha$ 's (see also Sect. 5.1).

\subsubsection{The actual role of $\gamma$}

One might now question in how far the underlying $g f$-distribution is of any importance for the final result, since it is much more the (relative) difference in abundance and especially in line number between iron group elements and light ions which leads to the "observed" line statistics. Accordingly, it is much more the mixture of different contributing ions with different ionization fractions and abundances, which plays a role, whereas the $g f$-dominated part of any specific ionization stage becomes visible only at the lowest end of contributing line-strengths.

To answer the above question, we have simulated the line-strength statistics resulting from different $g f$ distributions, again by Monte-Carlo, and compare the outcome with the actual situation in Fig. 22, both for the hotter and the cool wind. In contrast to the case of individual ions or to the case of uniform abundances (e.g., Figs. 15 and 17), the slope of the distribution in the decisive $k_{\mathrm{L}}>1$ range seems to be almost unaffected by the various $g f$-distributions, neglecting certain subtleties (e.g., the expected presence of small staircases for $\gamma \leq 1$ ) which are insignificant for any result derived from the cumulative distribution (Fig. 22, lower panel).

What really differs, is the vertical offset of the different distributions, where this offset is monotonically increasing for decreasing $\gamma$, and the actual case (asterisks) is met almost precisely for $\gamma$ between $\gamma=1.3\left(T_{\text {eff }}=10000 \mathrm{~K}\right)$ and $\gamma=1.2\left(T_{\text {eff }}=40000 \mathrm{~K}\right)$, in agreement with the average situation (Fig. 11). This behaviour is readily understood if we remember the discussion at the end of Sect. 4.2.2: In addition to controlling the slope of the weaker lines for individual ions, $\gamma$ controls the absolute line number $\Delta N$. In mathematical terms, this occurs via the $\gamma$-dependent normalization constant in Eq. (61) (note, that $w=w(\gamma)$ ). The physical interpretation is given in the discussion referred to: If the number of weak lines decreases for decreasing $\gamma$, the number of strong lines must consequently grow. This is the effect we observe in Fig. 22.

Thus, the final role of $\gamma$ is an important, however implicit one. Due to its relevance for the vertical offset and

8 The argument given here remains also valid for fluxweighted distribution functions, which are the actual basis for deriving force-multipliers. 

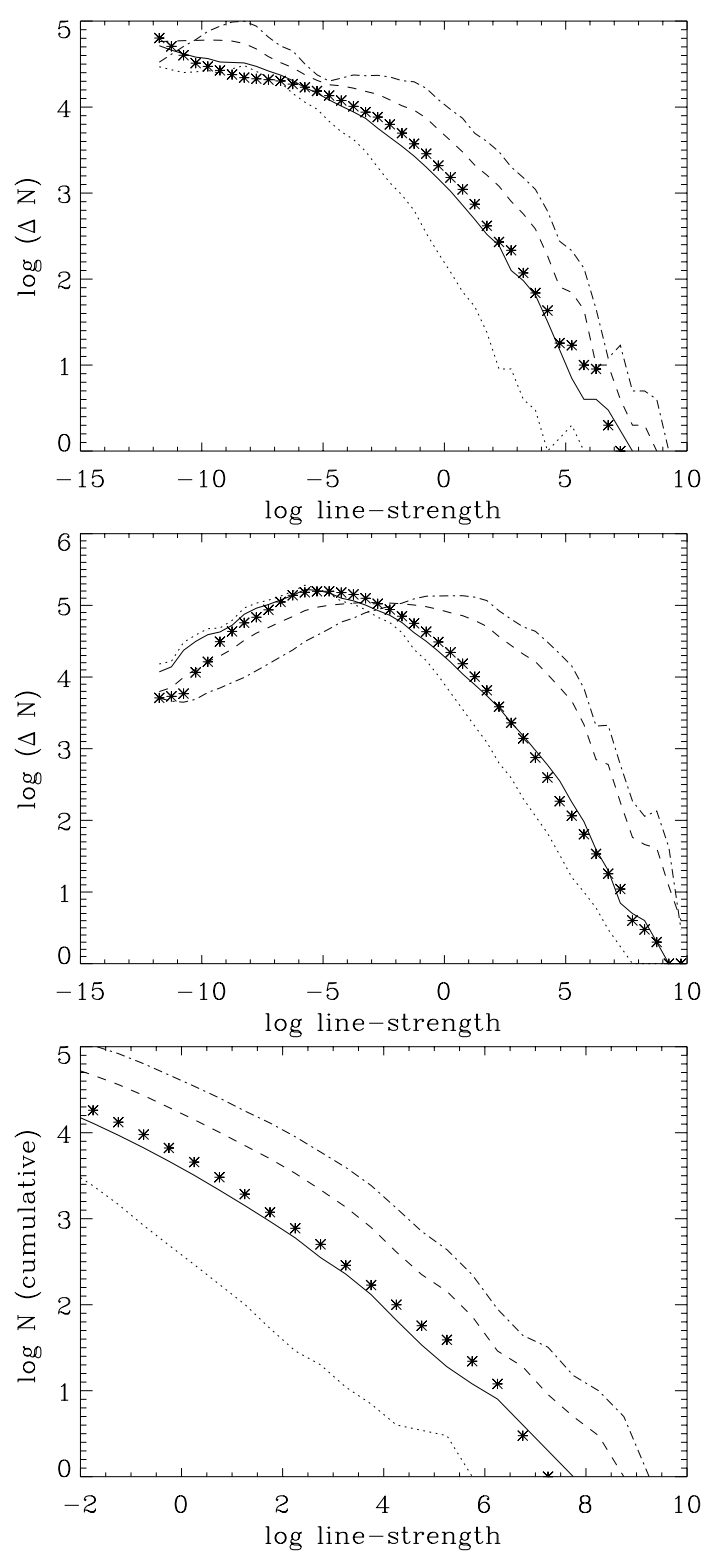

Fig. 22. Total line-strength distribution function for solar abundances, with different $g f$-distributions. Asterisks: actual case, lines: Monte Carlo simulation with constant exponent $\gamma=$ 1.5 (dotted), 1.0 (dashed) and 0.8 (dashed dotted). $r_{\max }=$ $2, r_{\min }=-6$. Upper panel $T_{\text {eff }}=40000 \mathrm{~K}, \gamma=1.2$ (fully drawn). Middle panel $T_{\text {eff }}=10000 \mathrm{~K}, \gamma=1.3$ (fully drawn). Lower panel: as upper one, however cumulative line number. The slope remains almost unaffected by any change in $\gamma$ !

with respect to derived force-multiplier parameters, it is much more decisive for the value of $k_{\mathrm{CAK}}$ (or $\bar{Q}$ ) than for the local slope $\alpha$ and consequently $\hat{\alpha}$.

4.2.8. Flux-weighted line-strength distribution functions and the difference of $\hat{\alpha}$ vs. $\alpha$

Our final task in order to describe the radiative line acceleration is to weight the line-strength distribution func- tions obtained so far by the appropriate flux distribution $L_{\nu} \nu / L$. This is done in Fig. 23, both for the model with equal as well as with solar abundances. With respect to the shape of the distribution, no dramatic effects are encountered, if we compare the non-weighted distributions (lines) with the corresponding flux-weighted ones (symbols). In terms of our discussion in Appendix E concerning the "saturation" effect, this is by no means surprising. The major impact of flux-weighting is at moderate line-strengths, where the weighted distribution becomes slightly flatter, since a number of high-frequency resonance lines of minor ions are blended out due to missing flux.

The corresponding force-multiplier parameters $\hat{\alpha}$ and $\hat{\delta}$ are displayed in Fig. 24 as iso-contours in dependence of $\log k_{1}$ and $\log \left(n_{\mathrm{e}} / W\right.$ ) (see Eq. (26) and the according derivative with respect to $\left.n_{\mathrm{e} 11} / W\right)$. Compared to our findings from the last section, nothing new has to be added: For solar composition, the resulting $\hat{\alpha}$ values are much more constant and larger than for the simulation with equal abundances. By inspection of the displayed values for $\hat{\delta}$, we find that they are rather small (much lower than the value of $1 / 3$ found for hydrogenic trace ions), indicating the dominance of major ionization stages and the frozen in ionization of stellar winds.

In order to account for more realistic fluxes, we have calculated additionally the case of an irradiation by Kurucz fluxes (consistently used also in the ionization equilibrium). Although some quantitative differences become visible (which turn out to be important for a correct description of B-star winds, cf. Petrenz 1999), the general effects are small and do not change any qualitative conclusion derived so far.

Since we have included now all ingredients required to calculate line-force and force-multiplier parameters, we can come back to one of the problems stated in Sect. 2, namely the difference of $\hat{\alpha}$ (derived from the lineacceleration itself) and the local slope of the flux-weighted distribution function, $\alpha$. In accordance with our analytical results from Sects. 2.3.2 and 2.4, Fig. 25 displays the following, by means of our $T_{\text {eff }}=40000 \mathrm{~K}$ model: For not too large $k_{1}$ respectively $k_{\mathrm{L}}$, both numbers are fairly similar. At the steep end of the distribution, however, where the local slope (symbols) becomes large (excitation dominated part of light ions) and $\alpha=1-s$ converges to small values (solar abundances) or values $\lesssim 0$ (equal abundances, $2 t / \sigma \approx 1$, cf. Sect. 4.2.6) the effective force-multiplier parameter $\hat{\alpha}$ remains positive (as it should, by definition). Thus, even at large $k_{1}, k_{\mathrm{L}}$ (i.e., in the outer wind part), the value of $\hat{\alpha}$ remains much more constant as if it were coupled to the local slope. Nevertheless, especially for equal abundances the decrease of $\hat{\alpha}$ at large line-strengths is significant! This decrease in $\hat{\alpha}$ can have severe consequences for low metallicity or thin winds, as we will see in the following section. 


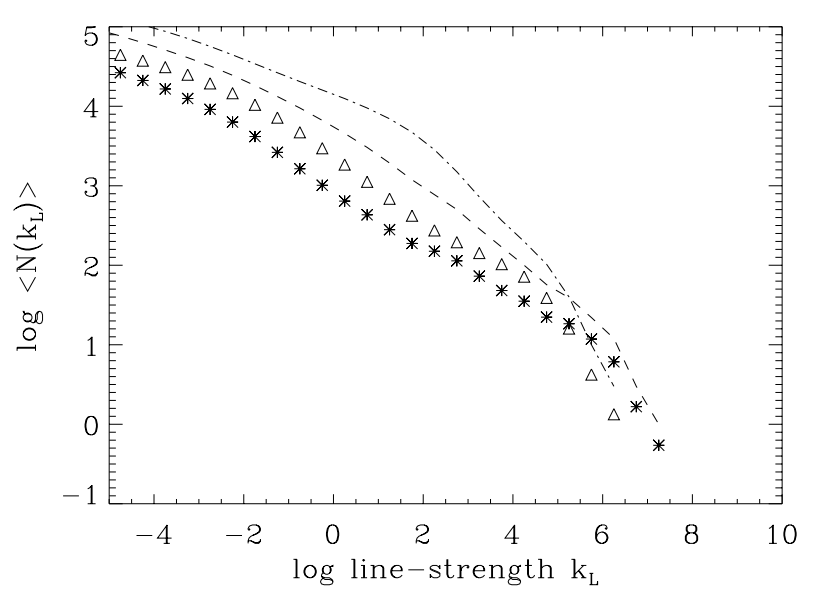

Fig. 23. Cumulative flux (times frequency) weighted linestrength distribution function for atmospheric parameters as in Fig. 14 and $T=40000 \mathrm{~K}$. Flux assumed to be Planck. Asterisks: solar, triangles: equal abundances. For comparison, the unweighted cumulative line-strength distribution functions (corresponding to the left panel in Fig. 21) have been overplotted. Dashed: solar, dashed-dotted: equal abundances
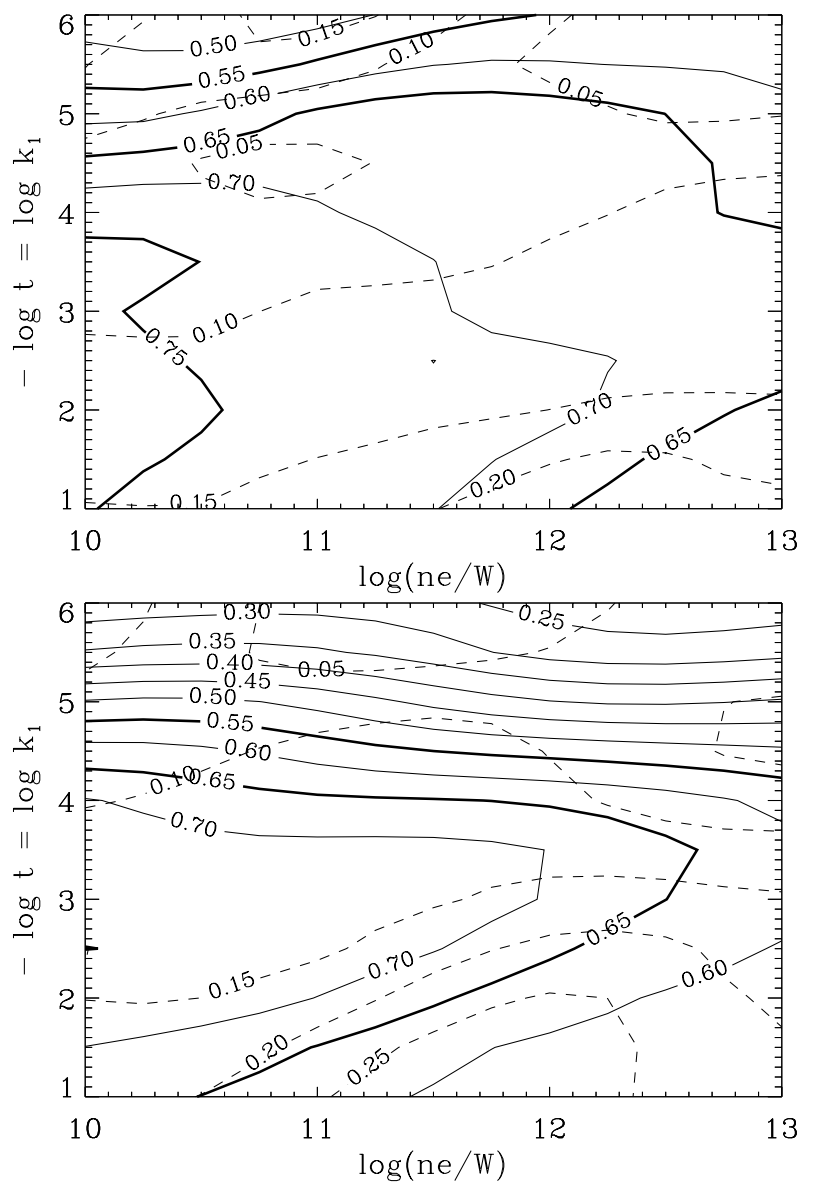

Fig. 24. Iso-contours of force-multiplier parameters $\hat{\alpha}$ and $\hat{\delta}$ as function of $\log \left(n_{\mathrm{e}} / W\right)$ and $-\log t=\log k_{1}$. Radiation field Planck, $T_{\mathrm{e}}=40000 \mathrm{~K}$, dilution factor $W=0.33$. Fully drawn: $\hat{\alpha}$, dashed: $\hat{\delta}$. Thick curves stress the values $\hat{\alpha}=0.55,0.65$ and 0.75. Upper panel: solar abundances; lower panel: equal abundances

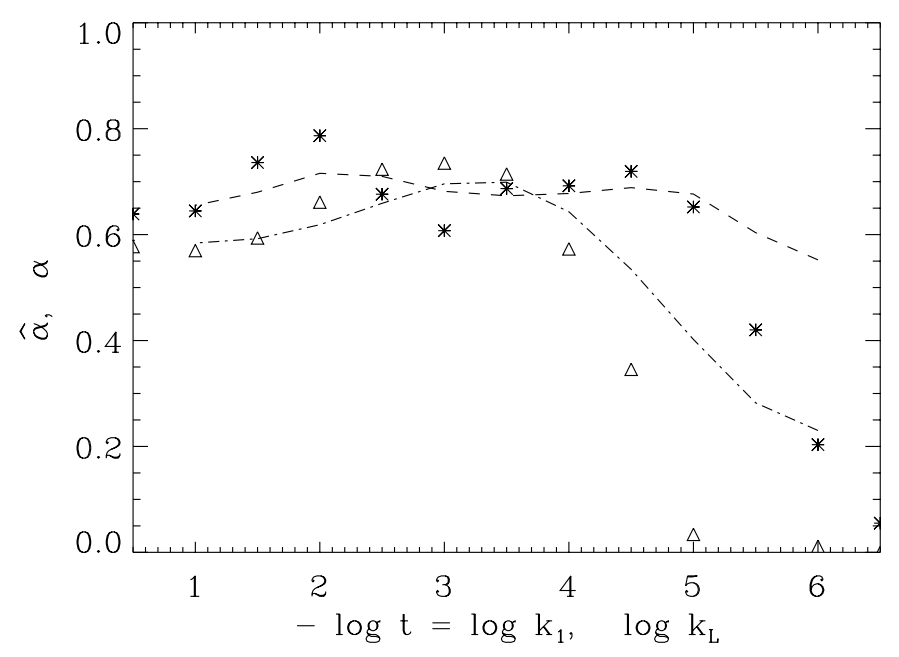

Fig. 25. Comparison of $\hat{\alpha}\left(k_{1}\right)$ and local slope of flux-weighted line-strength distribution function. Model as in Fig. 24, $\log \left(n_{\mathrm{e}} / W\right)=12$. Dashed: $\hat{\alpha}$ (solar), dashed-dotted: $\hat{\alpha}$ (equal abundances). Asterisks (solar) and triangles (equal abundances) give local slope as a function of $k_{\mathrm{L}}$, corresponding to the distributions displayed by similar symbols in Fig. 23

\section{Metallicity effects, thin winds and scaling relations}

Having understood the origin of the specific shape of the line-strength distribution function and its impact on the force-multiplier parameters in detail, we are now able to consider the question raised at the beginning of this paper, namely in how far the situation changes for different wind conditions. We will concentrate here on principal effects which are valid under fairly general circumstances. In particular, let us firstly consider the consequences if the overall metallicity is changed.

\subsection{The direct effect}

Due to its definition (6), the line-strength scales with metallicity (under the realistic assumption that the ionization balance is not severely modified) as

$k_{\mathrm{L}}(\epsilon) \sim z \frac{n_{1}(\odot)}{\rho} \sim z k_{\mathrm{L}}\left(\epsilon_{\odot}\right)$

where $z$ is the actual abundance $\epsilon$ relative to its solar value, $z=\epsilon / \epsilon_{\odot}$. Thus, the major effect of changing the metallicity is a shift of the according line-strength distribution functions (in the $\log -\log$ representation) to the "left" (for $z<1$ ) or to the "right" (for $z>1$ ).

Figure 26 verifies this behaviour for some exemplaric atmospheric conditions $\left(T_{\text {eff }}=40000 \mathrm{~K}\right.$ and $10000 \mathrm{~K}$, respectively) and three different metallicities, namely $z=1$ (solar), $z=0.1$ (roughly SMC) and $z=3$ (typical for the Galactic center). The shift to lower/higher line-strengths is clearly visible. Only for the largest line-strengths at $T_{\text {eff }}=10000 \mathrm{~K}$ the distributions seem to be unaffected 


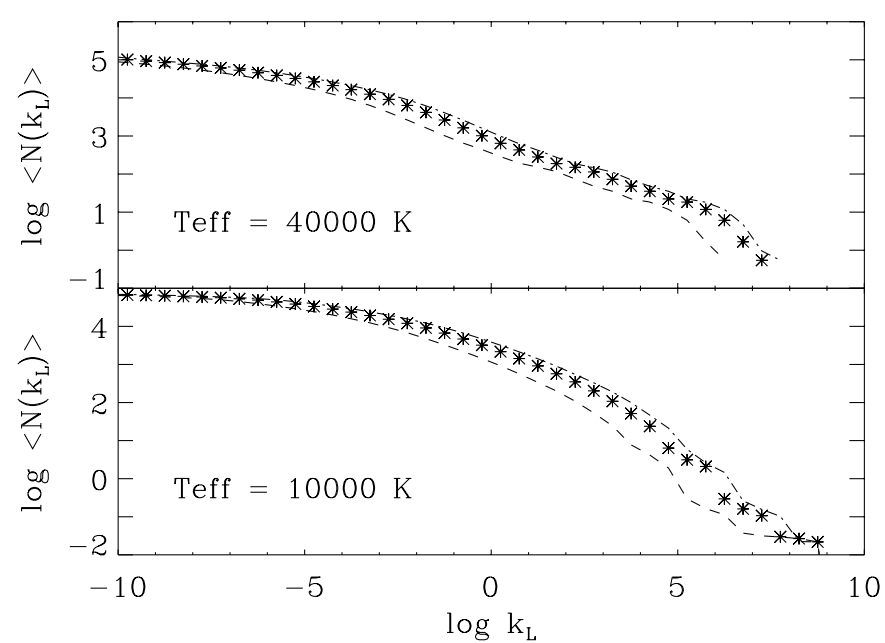

Fig. 26. Cumulative flux weighted line-strength distribution function for $T_{\text {eff }}=40000 \mathrm{~K}$ (upper panel) and $10000 \mathrm{~K}$ (lower panel), dilution factor $W=0.5$ and $n_{\mathrm{e}} / W=10^{12}$. Asterisks: solar abundance; dashed: $z=0.1$; dashed-dotted: $z=3.0$. Radiation field Planck

by metallicity, which is not surprising since the participating lines are transitions from the hydrogen Lyman series (cf. Sect. 4.2.6).

If we try to translate the shift in metallicity (affecting the independent variable $k_{\mathrm{L}}$ ) into the corresponding shift of the dependent variable $\left\langle N\left(k_{\mathrm{L}}\right)\right\rangle$, this relates to a modification of the vertical offset of the distribution, i.e., of the normalization constant, or, in other words, of the total number of contributing lines. In case of a perfect power-law then, the normalization varies according to

$$
\begin{aligned}
\mathrm{d} N\left(\nu, k_{\mathrm{L}}\right)(\epsilon) & =-N_{\mathrm{o}}(\epsilon) f_{\nu}(\nu) k_{\mathrm{L}}^{\alpha-2} \mathrm{~d} \nu \mathrm{d} k_{\mathrm{L}} \\
N_{\mathrm{o}}(\epsilon) & =N_{\mathrm{o}}\left(\epsilon_{\odot}\right) z^{1-\alpha} .
\end{aligned}
$$

Since the force-multiplier parameter $k_{\mathrm{CAK}}$ is proportional to $N_{\text {o }}$ (Eq. 12), it should scale according to

$k_{\mathrm{CAK}}(z) \sim z^{1-\alpha} \quad$ (perfect power-law).

With respect to $\bar{Q}$ and from its definition (37), obviously

$\bar{Q}(z) \sim z$

is predicted (cf. Gayley 1995), (almost) independent from the specific shape of the line distribution.

In Table 3 we have calculated the f.m. parameters for the same "models" as in Fig. 26. The last column shows the validity of the linear dependence $\bar{Q} \sim z$ for the hotter atmospheres, whereas for the cooler ones $\bar{Q}$ remains much more constant. If we remember that $\bar{Q}$ is dominated by lines of maximum strength (Sect. 2.6 and Appendix C), this behaviour results from the fact that the strongest driving lines in this temperature domain are those from hydrogen and thus remain rather unaffected by a change of global metallicity. Again, the conceptual simplicity of the $\bar{Q}$-approach is hampered by additional effects becoming obvious only by means of detailed calculations.
Table 3. Force-multiplier parameters as a function of $T_{\text {eff }}$ and metallicity $z$, for $n_{\mathrm{e}} / W=10^{12}$ and $W=0.5$. Values denoted with "(1)" derived from regression in the range $\log t=$ $-1 \ldots-6$ (as in Table 2 ); values with "(2)" refer to a range $\log t=-1 \ldots-5$ with optical depth parameter $t$

\begin{tabular}{rcccccr}
\hline \hline$T_{\text {eff }}$ & $z$ & $\log k_{\mathrm{CAK}}^{(1)}$ & $\hat{\alpha}(1)$ & $\log k_{\mathrm{CAK}}^{(2)}$ & $\hat{\alpha}(2)$ & $\bar{Q}$ \\
\hline 40000 & 3.0 & -0.98 & 0.68 & -0.98 & 0.69 & 5817 \\
& 1.0 & -1.12 & 0.67 & -1.15 & 0.69 & 1941 \\
& 0.1 & -1.28 & 0.62 & -1.42 & 0.67 & 196 \\
10000 & 3.0 & -0.41 & 0.47 & -0.52 & 0.52 & 997 \\
& 1.0 & -0.51 & 0.43 & -0.64 & 0.47 & 767 \\
& 0.1 & -0.87 & 0.36 & -0.96 & 0.40 & 663 \\
\hline
\end{tabular}

The other columns display the $k_{\mathrm{CAK}}$ and $\hat{\alpha}$ values derived by linear regressions to the calculated forcemultipliers, both in the range of $\log t=-1 \ldots-6$ (" 1 ") as well as in the range of $\log t=-1 \ldots-5$ ("2") with optical depth parameter $t=k_{1}^{-1}$. From the differences, it is immediately clear that the assumption of a more or less perfect power-law is only valid for the hotter atmosphere and low to intermediate line-strengths, consistent with the run of $\hat{\alpha}\left(k_{1}\right)$ shown in Fig. 27. Thus, the predicted scaling of $k_{\mathrm{CAK}}$ (Eq. 80) is only verified for case "2" at $40000 \mathrm{~K}$, whereas in all other cases the reaction of $k_{\mathrm{CAK}}$ is much weaker.

One should note, however, that the primary influence of $k_{\mathrm{CAK}}$ regards the definition of the mass-loss rate. Thus, $k_{\mathrm{CAK}}$ is most important in the subcritical region of the wind, where $k_{1}$ is low (and $t$ is large), and the contributing range in $k_{1}$ is also small (typically 2 dex). Under those conditions, however, a power-law distribution with $\hat{\alpha} \approx \alpha\left(k_{1}\right)$ can be always justified (cf. Sect. 2.3.2), so that the effective $k_{\mathrm{CAK}}$-value controlling the mass-loss rate should actually scale with (80), provided we compare winds of similar density. In so far, the variations displayed in Table 3 are an artefact of the much larger range of regression applied.

\subsection{The indirect " $\hat{\alpha}$ "-effect for low metallicity and thin winds}

Besides the obvious direct effect that the cumulative number of lines varies in concert with $z$, we have to account for an additional complication: By comparison of the various $\hat{\alpha}$ values derived by linear regression, we find that also $\hat{\alpha}$ is a function of metallicity, especially for cooler temperatures. Regarding the difference between actual (Fig. 27) and "power-law" fitted values, the depth-dependent values of $\hat{\alpha}$ are typically smaller than the mean for large $k_{1}$, whereas they are larger or similar at low $k_{1}$-values. (Due to the dominance of hydrogen lines with their $\alpha=2 / 3$ statistics, for the cooler atmosphere we even encounter an - otherwise untypical - steep increase of $\hat{\alpha}$ towards maximum $k_{1}$ ). 


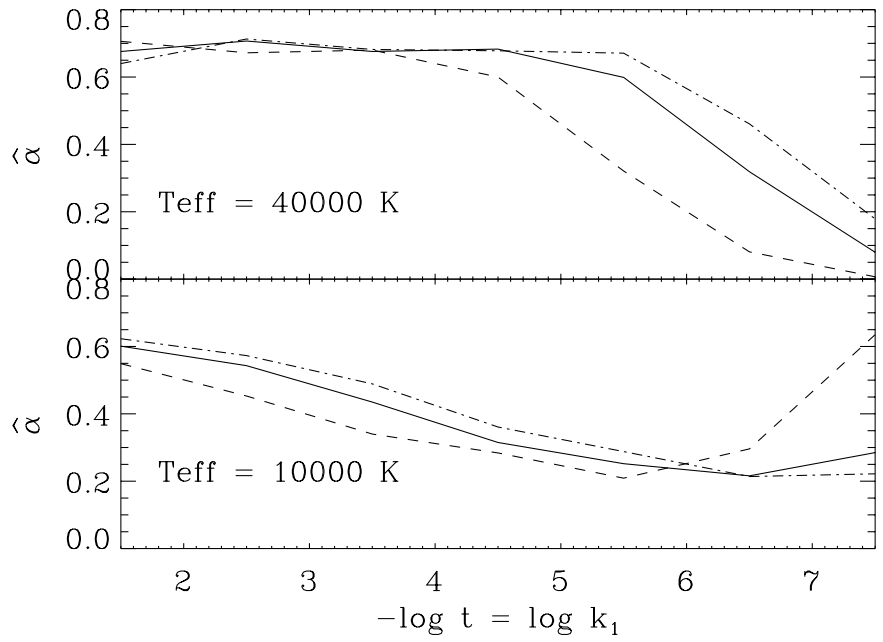

Fig. 27. As Fig. 26, however for $\hat{\alpha}$ (derived from forcemultiplier) as function of $k_{1}$. Solar abundances: fully drawn

The reason for the outlined behaviour is, again, the steep decline of the line-strength distribution at its upper end, due to the excitation effects discussed extensively in Sect. 4.2, and the horizontal shift of the distribution as a function of metallicity. Thus, for lower $z$ the steeper end of the distribution becomes visible at lower values of $k_{1}$, and $\hat{\alpha}$ can be roughly expressed as

$\hat{\alpha}\left(\log k_{1}, z\right) \approx \hat{\alpha}\left(\log k_{1}-\log z, z=1\right)$,

neglecting certain subtleties arising from non-metallic lines.

Whereas $k_{\mathrm{L}}$ is an (almost) density independent quantity, $k_{1}=\mathrm{d} v / \mathrm{d} r / \rho \propto v_{\infty}^{2} R_{*} / \dot{M}$ scales with the inverse of the mean wind density (times $v_{\infty} / R_{*}$ ). Thus, in addition to the metallicity shift (argument of rhs in Eq. (82)), the range of $\log k_{1}$ present in the wind is also shifted compared to solar conditions. Since a reduced metallicity yields a reduced wind density, this shift is towards higher $k_{1}$, i.e., a low-metallicity wind plasma "doubles" the effect of lowering $\hat{\alpha}$. In contrast, enhanced metallicities have almost no effect on $\hat{\alpha}$, since the corresponding shift is towards lower $k_{1}$, where the line-strength distribution function has a more constant slope.

Of course, the described process is also present if the wind-density is low for other reasons, e.g. because the luminosity is low. Compared to supergiant winds then, the $k_{1}$ range to be considered is shifted towards higher values, and $\hat{\alpha}$ is accordingly lower.

In conclusion, thin and fast winds as well as low metallicity winds tend to have lower $\hat{\alpha}$-values than high density or high metallicity winds, both on the average as well as locally. Once more, the reason for this effect is the curvature of the line-strength distribution function, especially at highest line-strengths, which is also the answer to the problem raised at the end of Sect. 2.5 concerning the origin of the lower $\hat{\alpha}$-values calculated in a low metallicity environment. If, on the other hand, a perfect power-law were present, $\hat{\alpha} \approx \alpha \approx$ const, independent on wind density and metallicity.

As a consequence of the variations of $\hat{\alpha}$ as a function of $k_{1}, \hat{\alpha}$ varies throughout the wind, since $k_{1}$ changes by typically three dex from inside to outside ${ }^{9}$. Hence, any exact hydrodynamic solution requires depth dependent forcemultipliers (cf. Kudritzki et al. 1998)

At this point of reasoning, we like to reiterate our findings in a somewhat different context. From our experience, the behaviour of the line-force in a low-density environment is frequently misinterpreted. E.g., after having calculated the according f.m. parameters - with the result of $\alpha \rightarrow 0$ in the outermost wind part -, there seems to be a common concern whether this is not only an artefact of an incomplete line-list at lower $g f$-values. Actually, however, almost the opposite effect is present! If, e.g. in Bdwarf winds, the density becomes so low that $\log t \lesssim-7$ (corresponding to $\log k_{1} \gtrsim 7$ ), all lines contribute to the line-acceleration at their optically thin limit, $g_{\mathrm{rad}} \propto \bar{Q}$. Thus, the strongest (however optically thin) lines have the largest influence and the numerical value of the total force does not depend on any incompleteness of the line list at low $g f$-values. The fact that $\alpha$ tends to zero in this case is, as explained already in Sect. 2.3, given by the independence of the line-force on any variation of $t$ (or $\left.k_{1}\right)$.

With respect to the horizontal shift "to the left" in a low-metallicity environment, this independence on $k_{1}$ can start even earlier, i.e., the line-force becomes saturated $(\alpha \rightarrow 0)$ at lower values of $k_{1}$. Even in cases of a higher metallicity (where the effects of an incomplete line-list may become obvious at least in principle), the actual range of contributing $k_{\mathrm{L}}$ values is normally much too small that this might become a real problem.

\subsection{Scaling relations}

Including now the aforementioned finite disk correction factor and accounting for ionization effects $N_{\mathrm{o}} \propto\left(n_{\mathrm{e} 11} / W\right)^{\delta}$, we can summarize the resulting scaling relations for $\dot{M}$ and $v_{\infty}$ as function of metallicity, which arise if a metal dependent line-force is used to solve the hydrodynamic equations (for actual solution methods, cf. PPK and Kudritzki et al. 1989) and the f.m. parameters were constant throughout the wind:

$$
\begin{aligned}
\dot{M} & \sim\left(z^{1-\hat{\alpha}}\right)^{\frac{1}{\alpha^{\prime}}}\left(\frac{L}{L_{\odot}}\right)^{\frac{1}{\alpha^{\prime}}}\left(\frac{M}{M_{\odot}}(1-\Gamma)\right)^{1-\frac{1}{\alpha^{\prime}}} \times \\
& \times g\left(k_{\mathrm{CAK}}(z=1), \hat{\alpha}, \alpha^{\prime}\right)
\end{aligned}
$$

${ }^{9}$ Only for the CAK velocity law with exponent $\beta=0.5$ this value remains constant, whereas the inclusion of the "finite disk correction factor (Friend \& Abbott 1986; PPK) as well as the observations (Puls et al. 1996) give considerably larger values $\beta=0.8 \ldots 1.3$ 


$$
\begin{aligned}
v_{\infty} & =\frac{\hat{\alpha}}{1-\hat{\alpha}} v_{\mathrm{esc}} f(\hat{\alpha}, \hat{\delta}) \\
\alpha^{\prime} & =\hat{\alpha}-\hat{\delta} \\
g & \approx\left(\frac{\hat{\alpha}}{1-\hat{\alpha}}\right)^{\frac{\hat{\alpha}}{\alpha^{\prime}}}\left(310^{-5} k_{\mathrm{CAK}}(1-\hat{\alpha})\right)^{\frac{1}{\alpha^{\prime}}} .
\end{aligned}
$$

$M$ is the stellar mass and $v_{\text {esc }}$ the escape velocity, corrected for the Eddington factor $\Gamma . f(\hat{\alpha}, \hat{\delta})$ is a decreasing function of $\hat{\delta}^{-1}$, and has a value of roughly 2.2 if $\hat{\delta}$ is small (cf. Kudritzki et al. 1989). The function $g$ finally accounts for the (moderate) dependence on terms of order $\hat{\alpha}$, on the proportionality to $k_{\mathrm{CAK}}^{1 / \alpha^{\prime}}$, and, most important (and frequently forgotten), on the scaling factor $\Gamma \approx 310^{-5}\left(L / L_{\odot}\right) /\left(M / M_{\odot}\right)$, since the mass-loss rate actually depends on the Eddington factor $\Gamma^{1 / \alpha^{\prime}}$ and not on $L^{1 / \alpha^{\prime}}$ itself. Note, that the variation of $g$ has to be considered in any comparison where $\hat{\alpha}$ is different (e.g., A-star vs. O-star winds, see below).

In case of depth dependent parameters, $\dot{M}$ relates to the conditions at the critical point $\left(\log k_{1}=2 \ldots 3\right.$ for not too thin winds), where $\hat{\alpha}$ and $\hat{\delta}$ do not vary heavily. The terminal velocity, however, is dependent on some average value of $(\hat{\alpha}, \hat{\delta})$ between the location of the critical point and large values of $k_{1}$, and will be typically smaller compared to using the $\hat{\alpha}$ values present at the critical point, because of the reasons outlined above.

In any case, to first order we find the metallicity effect as

$\dot{M} \sim z^{\frac{1-\hat{\alpha}}{\alpha^{\prime}}} ; v_{\infty} \sim \frac{\hat{\alpha}}{1-\hat{\alpha}}(z)$

which, in case of $O$-star winds (small $\hat{\delta}$ ) yields the often quoted scaling relation for the mass-loss rate $\dot{M} \sim \sqrt{z}$ since $\hat{\alpha} \approx 0.6$.

Due to the metallicity dependent factor $\hat{\alpha} /(1-\hat{\alpha})$, one can expect lower terminal velocities in a metallicitydeficient environment. This is just what has been found by comparing O-star terminal velocities in the Galaxy and the Clouds, cf. Fig. 28. For a detailed discussion, we refer the reader to the papers by Garmany \& Conti (1984), Kudritzki et al. (1987), Haser et al. (1993) and Walborn et al. (1995).

Finally and with respect to the wind-momentum luminosity relation (Kudritzki et al. 1995; Puls et al. 1996), our findings imply (leading terms only)

$$
\begin{aligned}
& \log D_{\mathrm{mom}}=\log \left(\dot{M} v_{\infty}\left(R_{*} / R_{\odot}\right)^{\frac{1}{2}}\right) \sim \\
& \sim \frac{1}{\alpha^{\prime}} \log L / L_{\odot}+\frac{1-\hat{\alpha}}{\alpha^{\prime}} \log z+2 \log \left(\frac{\hat{\alpha}}{1-\hat{\alpha}}\right)+ \\
& +\frac{1}{\alpha^{\prime}} \log \left(310^{-5} k_{\mathrm{CAK}}(z=1)(1-\hat{\alpha})\right)+\ldots,
\end{aligned}
$$

where, of course, in case of $\hat{\alpha}(z !) \neq 2 / 3$ an additional correction for mass effects might be necessary.

From the presently available data, it is clear that at least in the SMC a different offset is visible (due to the

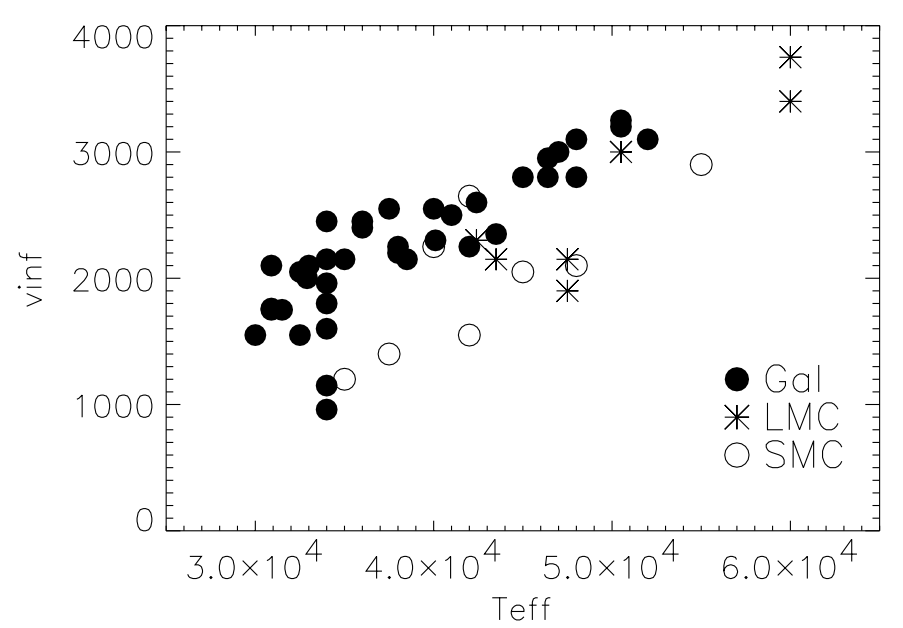

Fig. 28. Terminal velocities of O-type stars in the Galaxy and the Magellanic clouds. Data from Haser (1995) and Puls et al. (1996)

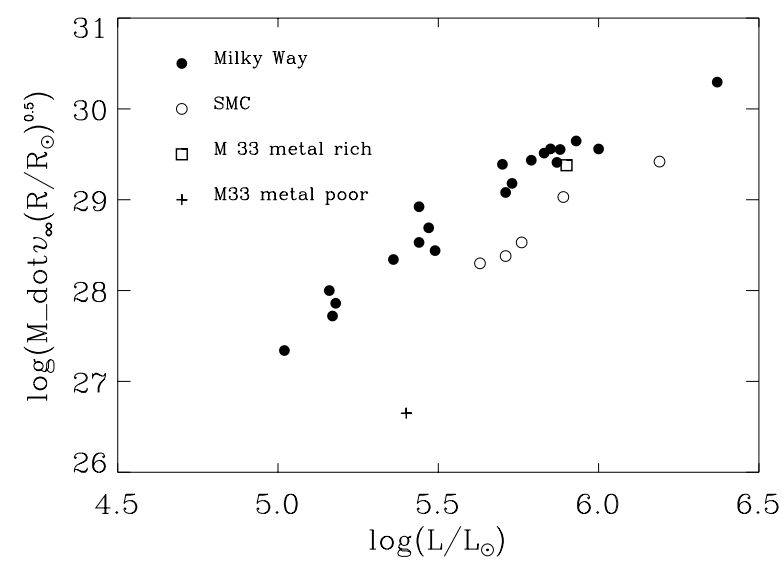

Fig. 29. Wind momentum (in cgs units) and luminosity of galactic and SMC supergiants and two A-supergiants in M 33. Open square: M 33 A-supergiant with galactic metallicity. Cross: Extremely metal poor A-supergiant in the outskirts of M 33. (From McCarthy et al. 1995)

second term in the above equation, resulting from the "direct" effect (cf. Fig. 29, and also Puls et al. 1996; Kudritzki 1997). Whether there is actually a different slope (as a consequence of a reduced $\alpha^{\prime}$ ), is not certain due to the small number statistics for SMC O-stars. To clarify the situation, more objects have to be analyzed. This work is well under way in our group.

Contrasted to the above uncertainty concerning the reduction of $\hat{\alpha}$ in a metal poor environment, the observational status quo with respect to the difference of A-star vs. O-star winds is much more promising. From the latest results by Kudritzki et al. (1999), the WLR for Galactic A-Supergiants (in a temperature range $T_{\text {eff }}=8400 \ldots 9400 \mathrm{~K}$ ) reads $\log D_{\text {mom }}=0.38^{-1} \log L / L_{\odot}+14.22$, to be compared with 
the relation valid for Galactic O-Supergiants (from Puls et al. 1996), $\log D_{\text {mom }}=0.65^{-1} \log L / L_{\odot}+20.40$.

Note at first that the observed slope (interpreted as $\alpha^{\prime}=\hat{\alpha}-\hat{\delta}=0.38$ ) lies exactly in the range to be expected for A-type winds, cf. Tables 2 and 3. Second, from the difference in the offset compared to O-stars $\left(\Delta \log D_{\mathrm{o}}=6.18\right)$, we can calculate the average value of the parameter $k_{\mathrm{CAK}}(\mathrm{A}-\mathrm{SG})$ using Eq. (88), the values found for $\alpha^{\prime}$ from the WLRs and the appropriate values for $\hat{\alpha}$ and $k_{\mathrm{CAK}}(40000 \mathrm{~K})$ from Table 3 . In result, we find $\log k_{\mathrm{CAK}}(\mathrm{A}-\mathrm{SG})=-0.84$. This number is reasonable when compared to our theoretical prediction $\log k_{\mathrm{CAK}}(10000 \mathrm{~K})$ $=-0.51$ (Table 3 ) at $n_{\mathrm{e}} / W=10^{12}$, accouting for the fact that A-Supergiants have lower densities at the critical point (smaller $\dot{M}$ and larger radii) than their O-type counterparts. In so far, the difference found is a consequence of the $\delta$-term, which is implicitely included in the value of $k_{\mathrm{CAK}}$ derived in our comparison.

Thus, we conclude that our theoretical predictions concerning the run of $\hat{\alpha}$ (and $k_{\mathrm{CAK}}$ ) with respect to temperature are correct, and, additionally, in Sect. 4 we have explained the reason for this behaviour.

\section{Summary and discussion}

In this paper, we have tried to analyze various aspects concerning the relation between line-statistics and radiative driving in massive stars with not too thick winds (i.e., we have excluded the problem of WR winds).

In the following, we will summarize our results, point to additional aspects which have not been discussed in the previous sections and give some caveats where necessary.

In Sect. 2, we found an alternative interpretation of the total line-force as the integral of the cumulative flux weighted line-strength distribution function over linestrength, which allowed for an instructive visualization of the line-force and further investigations: For arbitrary distribution functions, the local logarithmic slope $\alpha\left(k_{\mathrm{L}}\right)^{10}$ can be identified with the f.m. parameter $\hat{\alpha}\left(k_{1}\right)$, if this slope is not too steep around $k_{\mathrm{L}}=k_{1}$ (essentially, $\alpha$ has to be larger than zero). If the latter condition is violated locally, $\hat{\alpha}$ should follow at least the basic trend of $\alpha$, however remaining larger/equal than zero with respect to its definition as the ratio of optically thick to total line acceleration. (A steep increase with $\alpha<0$ over a larger range of line-strength well below $k_{\mathrm{L}}=k_{1}$, finally, would prohibit the parameterization of the f.m. in its usual form $\propto k_{1}^{\hat{\alpha}}$ completely.) These statements were checked for various conditions throughout the paper, and turned out to be fulfilled always.

In order to understand the principal behaviour and numerical value of $\hat{\alpha}$ itself and to allow for predictions con-

10 Actually, $\alpha$ is not the local slope itself, however $\alpha=1-s$, if $s$ is the negative exponent of the according cumulative distribution function. cerning its behaviour under different conditions, we have performed a rigorous discussion of the line-strength distribution as function of atmospheric conditions. This discussion relied on our extensive data base and an approximate NLTE description, provided in Sect. 3.

For some typical atmospheric conditions, we checked at first the applicability of Gayley's (1995) $\bar{Q}$-approach. As long as the dependence on the maximum line-strength $Q_{\mathrm{o}}$ is correctly accounted for, a perfect consistence with the older CAK approach using the parameter $k_{\mathrm{CAK}}$ is found. We concluded, however, that the $\bar{Q}$ formalism is only advantageous in those cases when the maximum linestrength $Q_{\mathrm{o}}$ is of the same order as $\bar{Q}$, a prerequisite which was assumed by Gayley to be valid always. By means of our detailed calculations, however, this assumption could be validated only for hot winds $\left(T_{\text {eff }} \gtrsim 35000 \mathrm{~K}\right)$. For cooler ones, the difference between $\bar{Q}$ and $Q_{\mathrm{o}}$ is significant and was attributed to the increasing mismatch between the frequential positions of the strongest lines and flux-maximum. In those cases, a "blind" application of the final scaling relations provided by Gayley (which include the assumed equality) will inevitably lead to erroneous conclusions.

In Sect. 4, we turned to the central question concerning the slope (or shape) of the line-strength distribution function. At first, we considered the (simpler) case of hydrogen (or hydrogenic ions) and derived the important result that the according slope in the essential $k_{\mathrm{L}}$ range is almost exactly $-1 / 3$, so that $\alpha=2 / 3$. This result was shown to be the final consequence of the corresponding oscillator strength distribution, in particular the dependence $g f \propto n^{-3}$ with $n$ the principal quantum number of the upper level of the contributing transitions. The predicted behaviour is actually seen at the hydrogen-Lyman dominated, high line-strength end calculated for distribution functions under A-type conditions. Additionally we showed that, if neutral hydrogen is a trace ion, the corresponding $\delta$ term (resulting from the $n_{\mathrm{e}} / W$ dependent part of the normalization constant $N_{\mathrm{o}}$ ) is of order $1 / 3$, and that for arbitrary trace ions one stage below the major one the equality $\alpha+\delta \approx 1$ should hold in general.

In order to derive line-strength distribution functions for arbitrary metallic ions, we followed the approach suggested by Allen (1966), modified for the inclusion of nonuniform oscillator strength distributions. This approach and the above one are mutually exclusive, due to the rather specific behaviour of level density as function of energy in hydrogenic ions.

At first, we considered the so-called line-intensity distribution, which is the LTE analogon to (NLTE-)linestrength distribution functions for specific ions. By a number of Monte-Carlo simulations for the oscillator strength distribution we have convinced ourselves that the principal description, resulting in predictions of $\Delta N$ as function of line intensity, is valid both for the frequency 
dependent as well as the frequency integrated line intensity distribution.

We showed that three different slopes are possible, namely $2 t / \sigma, t / \sigma$ and $\gamma-1$, if $t$ is the temperature in units of $625 \mathrm{~K}, \sigma$ is the slope of the level-density with respect to energy and $\gamma$ the negative exponent of the differential oscillator strength distribution function. In dependence of $\gamma$ being larger or smaller than a critical value $\gamma_{\text {crit }}=1+2 t / \sigma$, specific predictions for the line intensity distribution functions can be made, which depend uniquely on the properties of the level- and line-lists. Thus, by comparing these analytical results with actual distribution functions, it is very easy to test for the completeness of the underlying data base of specific ions.

Under "normal" conditions, the resulting frequency integrated distribution function consists of two regimes, namely a steeper, excitation-dominated one with slope $2 t / \sigma$, and a second one with slope $\gamma-1$. The division is given at a line intensity $l=x_{\max } / t-\log g f_{\max }$, if only those lines with a lower energy level below a certain cutoff energy $x_{\max }$ are considered. Thus, the direct influence of $\gamma$ regards only the slope of the weaker lines' distribution. Additionally, however, $\gamma$ has an indirect impact which turns out to be of major relevance for the final result: By affecting the normalization constant of the line intensity distribution, it controls the absolute number of lines as function of intensity (or strength).

By translating our findings from line intensity to linestrength distributions, two important points have to be considered: The final distribution function consists of a number of contributing ions, so that the product of ionization-fraction times relative abundance becomes important. Second, under stellar wind conditions, NLTE effects have to be accounted for.

In order to separate NLTE/ionization- from abundance effects, we investigated at first the case of equal abundances and considered two groups of elements, namely "iron group" and "light" elements, respectively, which should behave rather similarly amongst each other due to their similar electronic structure. NLTE effects were treated in our approximate way by allowing for only three participating classes of lines, namely those with a lower ground- or meta-stable level and those lines directly connected to the former.

Under these assumptions, it turned out that the major difference between line intensity and -strength distribution is (in the log-log representation) a horizontal shift due to ionization, and the appearance of a low effective value for $x_{\max }$, giving rise to a rather narrow excitation dominated range. Within the specific subgroups, the individual ions behave rather similarly, so that the total distribution functions can be described in fairly simple terms ("staircase structure"), since only two or three different ionization stages contribute to the interesting range (six to eight dex) in $k_{\mathrm{L}}$. Iron group elements display a significant increase in line number with decreasing temperature due to their in- creasing complexity in electronic structure, whereas light ions show a comparable number of lines for all considered temperatures. The excitation dominated slope was found to be of order unity for both subgroups, independent of temperature, and relates to the increase of $\sigma$ (lower level density) with increasing ionization stage. Additionally, the maximum value of $k_{\mathrm{L}}$ reached by iron group elements is smaller than for light ions (lines to meta-stable levels vs. lines to ground-states).

The latter effect - difference in maximum line-strength between iron-group and light ions - is essentially increased if one accounts for (relative) solar abundances. Thus, at large line-strengths the resulting total distribution is determined by transitions from light ions (plus hydrogen at cooler temperatures), whereas for intermediate and lower $k_{\mathrm{L}}$ the iron group elements (most important: Fe itself due to its abundance) dominate the distribution. Consequently, at highest line-strengths the distribution is rather steep (excitation dominated part of light ions' distribution), however smaller than unity due to the variety of abundances present. The intermediate region is primarily controlled by the difference in line number between both groups and by the temperature dependence of the iron group contribution (as mentioned above, the light ions' distribution function remains rather constant): For hotter temperatures, there are fewer iron group lines, thus the slope is smaller and $\alpha$ accordingly higher; for lower temperatures, the iron group line number is much larger, inducing a larger slope and smaller $\alpha$. This explains the decrease of $\alpha$ (and $\hat{\alpha}$ ) with decreasing temperature. At lowest line-strengths $\left(k_{\mathrm{L}} \leq 0\right)$, finally, the oscillator strength distribution dominates the slope. The staircaselike structure "observed" for equal abundances is smeared out by the actual abundance pattern.

The deeper wind region is controlled by the linedistribution at lower $k_{\mathrm{L}}$ values. Thus, the mass-loss rate follows the radiative acceleration by iron group elements. Since increasing $k_{\mathrm{L}}$ means also increasing distance from the star, the outer velocity law and especially the terminal velocity is controlled by light ions ${ }^{11}$. Here, details of the distribution (e.g., decreasing $\hat{\alpha}$ ) are essential for a quantitative description.

Our results explain easily the contribution of various elements to the depth dependent force-multiplier as shown by Pauldrach (1987, Fig. 10). They also explain the extreme sensitivity of terminal velocities on subtle effects (small variations in temperature, density and composition, cf. Pauldrach et al. 1990, Fig. 8) having a decent influence on the light ions distribution function. Note that only a small number of lines affect the acceleration in the outer

\footnotetext{
11 After this study has been finished, we received a preprint by Vink et al. (1999) who have investigated the so-called bistability jump in hot star winds. By calculating the forcemultipliers in a way similar to our approach, they reached the same conclusions concerning the relative importance of iron group elements vs. light ions with respect to $\dot{M}$ and $v_{\infty}$.
} 
part of the wind! In so far, the large observed variance in $v_{\infty} / v_{\text {esc }}$ for O-star Supergiants (Howarth \& Prinja 1989) is not surprising at all.

We have stressed the importance of relative abundances, esp. with respect to the their mean value for the two groups of elements. If there were no differences, the line distribution would be much more curved (and the corresponding slopes more depth- and temperaturedependent) compared to solar conditions. Note that different mean values (e.g., in Pop. III stars) might result in wind properties which are significantly different from "present-day" objects.

Regarding the total distribution function, the direct influence of $\gamma$ concerning the slope (as discussed above for the cases of hydrogenic or individual ions, respecively) is almost completely lost. (Only at weak line-strengths $\left(\log k_{\mathrm{L}}<0\right)$, which are marginally contributing to the line-force, the $\gamma-1$ slope becomes visible again.) We have seen that a variety of different oscillator strength distributions lead to almost the same shape of the resulting line-strength statistics. What survives, however, is the indirect influence: The lower the value of $\gamma$, the more lines are present in the decisive $k_{\mathrm{L}}$ range! Thus, $\gamma$ controls the vertical offset of the distribution, or, in other words, $k_{\mathrm{CAK}}$ respectively $\bar{Q}$. By comparing with the actual case, it turns out that the effective value of $\gamma$ is of order 1.2 to 1.3 , i.e., is similar to the corresponding value for hydrogenic ions, although the specific value for certain ions can be different (e.g., FeIv has $\gamma \approx 1$ ).

Since the line acceleration results from the flux (times frequency) weighted line-strength distribution, we have briefly shown the influence of the corresponding operation (cf. also Abbott 1982). Although some differences between flux-weighted and "normal" distribution become visible and turn out to be of importance for quantitative calculations under specific conditions, the principal behaviour and all prior conclusions are not affected:

Unfortunately and contrasted to the case of hydrogenic ions which are controlled almost exclusively by the underlying oscillator strength statistics, there is no single process which dominates the final shape of the total line strength distribution. Summarizing our above results, the most important properties which have to be considered instead are

- the horizontal offset in the distribution of light and heavy ions, as function of relative abundance;

- the different number of lines from both groups and their specific dependence on temperature;

- the fact that the distribution at its high $k_{\mathrm{L}}$ end is controlled by excitation effects for the predominant ion species;

- the total line number depending on the (average) oscillator strength distribution.

As a consequence of these effects, we have explained the following properties which should be valid under typical conditions (relative abundances are solar): $\hat{\alpha}\left(k_{1}\right)$ decreases with decreasing $T_{\text {eff }}$, increasing $k_{1}$ and decreasing global metallicity $z$,

where the latter effect relies on the argument that a change in $z$ simply shifts the line-strength distribution horizontally. From these considerations, it is obvious that low metallicity and/or low density winds (from dwarfs) should have a smaller average $\hat{\alpha}$ than high metallicity and denser winds, with the implication of lower terminal velocities and a steeper wind-momentum luminosity relation. Present observations are consistent with any of the above theoretical considerations.

In most cases, the variation of $\hat{\alpha}$ (and the corresponding $\hat{\delta}$ ) throughout the wind is essential and has to be accounted for at least in consistent hydrodynamical solutions aiming at a quantitative description; the usual powerlaw with constant $\hat{\alpha}, \hat{\delta}$, however, may be justified in hotter (O-star) winds which tend to have more constant f.-m. parameters than cooler ones.

In view of this summary, one might question about the central ingredients which are inevitable to allow for a radiation force $\propto k_{1}^{\hat{\alpha}}$, with $\hat{\alpha}$ a rather constant function over the contributing range of $k_{1}$. Most important to this regard is the requirement that the slope of the line-distribution function is not to steep over several dex in line-strength. Otherwise, the line-force can no longer be described in the presumed way, and all scaling relations derived here or elsewhere would change. (E.g., Gayley 1995 considered a Gaussian line-strength distribution as an "academic" example.) Thus, a different world might be possible only if the $g f$-distribution were much steeper as it actually is and the level-density were much higher. If only the first condition were met and $\sigma$ reasonably large, the distribution would be controlled by $2 t / \sigma$ globally, since $\gamma>\gamma_{\text {crit }}$, and $\alpha=1-2 t / \sigma>0$ again. If, on the other hand, $\sigma$ were much smaller, however $\gamma$ as it is, no dramatic effects are to be expected since $x_{\max }$ remains small, as a final consequence of the dilution of the radiation field and density in an expanding medium.

Thus, although the shape of the line-distribution is determined by various processes, it would require a significantly different atomic physics (or coupling constants) in order to prevent radiation driven winds to behave as we think they do.

We finish this paper with one important comment. Although our results for line-strength distribution functions are valid under rather general circumstances (at least, if one accounts for a consistent description of the ionizing and illuminating radiation field), the performed transition to radiative accelerations (and scaling laws!) presumes at least two conditions to be valid:

First, the effects of line-overlap should be marginal or at least describable by some (almost) depth-independent correction factor (applied to $k_{\mathrm{CAK}}$ or $\bar{Q}$, e.g. Puls 1987). In case of stratified ionization structures with increased efficiency of multi-line scattering, which are attributed to 

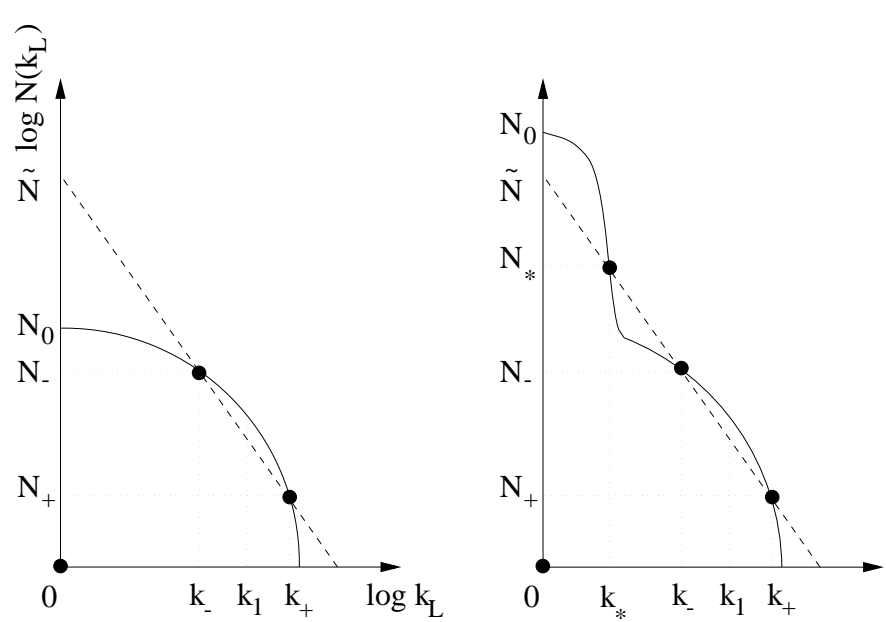

Fig. A1. Schematic sketch of two different kinds of line-number distributions, case A (left) and B (right). Note the log-log representation necessary to derive the local slope $\alpha-1$, in contrast to the linear representation of Fig. 1 required to calculate the line acceleration. Note also that the displayed minimum line-strength is $\log k_{\mathrm{L}}=0$. For an explanation of the various symbols, see also Sect. 2.3.2

be responsible for the large observed performance numbers of WR-winds (Lucy \& Abbott 1993; Springmann \& Puls 1998), additional considerations are required, including the role of the optically thick continuum in the wind. Note, however, that Gayley (1995) pointed to the formal possibility of inducing large $\dot{M}$ by decreasing the value of $\hat{\alpha}$ while, e.g., keeping the "standard assumption" of $Q_{\mathrm{o}} \approx \bar{Q} \approx 2000$. In our perspective, this might be feasible if the number of lines from iron group elements were essentially increased in the sub-critical region, e.g. if additional strong lines from excited levels were present.

Second, the prediction that thin winds should exhibit a smaller average $\hat{\alpha}$ value with accordingly modified scaling relations may describe only part of the actual situation. Due to the importance of velocity curvature terms in the transonic region (neglected in any CAKlike hydrodynamical approach), additional effects may be present which further reduce the mass-loss rate (cf. Owocki \& Puls 1999).

\section{Appendix A: Line force and local slope}

In this appendix, we will clarify the question under which circumstances the local slope of (generalized) line-strength distribution functions can be equalized to the CAK force multiplier $\alpha$. Following Eq. (24) in Sect. 2.3.2, this is possible under the condition

$$
\left|\frac{\alpha \bar{N}_{\left\{0, k_{-}\right\}}}{N_{+}}\left(\frac{k_{1}}{k_{+}}\right)^{1-\alpha}-\left(\frac{k_{1}}{k_{-}}\right)^{1-\alpha}\right| \ll \frac{k_{1}}{k_{-}},
$$

which depends strongly on the average cumulative line number $\bar{N}_{\left\{0, k_{-}\right\}}$(cf. Eq. 25). To proceed further, we have to investigate two cases. Case A, which is the more realistic one (cf. Sect. 4), comprises a situation where the linenumber distribution has a monotonic curvature in the log, corresponding to an increase of $\alpha$ for decreasing $k_{\mathrm{L}}$. This situation is sketched on the left of Fig. A1: Both the total line number $N(0)$ (as well as $N\left(\log k_{\mathrm{L}}=0\right.$ ) denoted by $N_{0}$ in our plots) and the average number of lines $\bar{N}_{\left\{0, k_{-}\right\}}$ lie well below the extrapolated value $\tilde{N}$. In this case, it is straightforward to show that the lhs of (A1) obtains its maximum value for the smallest value possible for $\bar{N}_{\left\{0, k_{-}\right\}}$, which is $N_{-}$. Using this value, the inequality becomes

$|\alpha-1| \ll\left(\frac{k_{1}}{k_{-}}\right)^{\alpha}$,

which under the considered circumstances can be (almost) always fulfilled as long as $\alpha>0$ ! Thus, for monotonically curved but otherwise unconstrained $\log N\left(k_{\mathrm{L}}\right)$ distributions (case A), the ensemble line acceleration follows the local (however not necessarily constant) slope of the fluxweighted and cumulative line-strength distribution function, as long as this is larger than -1 .

Case B (right panel of Fig. A1) displays the situation of a sharply increasing line number below a certain threshold value $k_{*}$. The asymptotic fit value $\tilde{N}$ is here significantly smaller than the actual value $N(0)$ and the average value $\bar{N}_{\left\{0, k_{-}\right\}}$. In this case, we define $N_{*}$ as the number of lines where the actual distribution and the fitted one cross each other, at line-strength $k_{*}$. To obtain an upper limit for our inequality (A1), we use the maximum possible value for $\bar{N}_{\left\{0, k_{-}\right\}}$,

$\operatorname{Max}\left(\bar{N}_{\left\{0, k_{-}\right\}}\right) k_{-}=N(0) k_{*}+\int_{k_{*}}^{k_{-}} N_{+} k_{+}^{1-\alpha} k_{\mathrm{L}}^{\alpha-1} \mathrm{~d} k_{\mathrm{L}}$, which after some algebra leads to the requirement

$N(0) \ll N_{*}\left(1+\left(\frac{k_{1}}{k_{*}}\right)^{\alpha}\right)$.

This requirement can be usually fulfilled if $k_{1}$ is large compared to $k_{*}$ (i.e., the sharp increase of line number occurs at relatively small line-strength), and, again, if $\alpha$ is positive.

In summary, we have shown that the CAK representation $g_{\text {rad }}^{\text {tot }} \propto k_{1}^{\alpha}$ with $\alpha$ corresponding to the local slope of the line-strength distribution function is valid under fairly general circumstances, if the slope is not too steep in the region around $k_{1}$, i.e., $0<\alpha \leq 1$ locally. Of course, if the distribution function is curved, this leads immediately to depth dependent force-multiplier parameters.

Finally, it is important to realize that our derivation has required some knowledge of the behaviour of optically thin lines, however did not constrain the distribution of optically thick lines in any respect. This, of course, is related to the fact that all optically thick lines behave similarly. Thus, the slope of the distribution for $k_{\mathrm{L}}>k_{1}$ is of no concern as long as we know the actual number of these lines. 


\section{Appendix B: Line-strength distribution with exponential cutoff.}

In a number of publications related to the calculation of the line-force instability (e.g., Owocki et al. 1988, however also Gayley 1995), a slightly modified version of the linestrength distribution function (10) has been used in order to prevent the instability from growing at wavelengths below the numerical grid resolution of order "Sobolevlength" $L_{\mathrm{S}}=v_{\mathrm{th}} /(\mathrm{d} v / \mathrm{d} r)$. To this end, an exponential cutoff at line-strength $k_{\max }$ has been introduced, where $k_{\max }$ can be considered as the maximum line-strength present or treated in the wind. Another argument for introducing this cutoff is given by the requirement of preventing the number of strong lines from becoming smaller than unity, i.e., only one line stronger than $k_{\max }$ shall be allowed to be present. The corresponding distribution with otherwise constant exponent $a$ - reads

$\mathrm{d} N\left(\nu, k_{\mathrm{L}}\right)=-N_{\mathrm{o}} f_{\nu}(\nu) k_{\mathrm{L}}^{a-2} \exp \left(-k_{\mathrm{L}} / k_{\max }\right) \mathrm{d} \nu \mathrm{d} k_{\mathrm{L}}$,

and the resulting line acceleration is modified by an additional factor

$g($ cut $)=g_{\text {rad }}^{\text {tot }}\left(\right.$ Eq. 18) $\times\left[\left(1+\frac{k_{1}}{k_{\max }}\right)^{1-a}-\left(\frac{k_{1}}{k_{\max }}\right)^{1-a}\right]$

which for $k_{1} / k_{\max } \rightarrow 0$ approaches unity, of course. Note, that $k_{1} / k_{\max }=1 / \tau_{\max }$ relates to the maximum allowed optical depth per line at the considered depth point. It is interesting to investigate for this type of line-strength distribution the ratio of optically thick to total line-force, corresponding to our definition of $\hat{\alpha}$ in Sect. 2.4. For large values of $k_{\max }$, compared to $k_{1}$, this quantity should resemble the input value of $a$. However, for $k_{1}$ approaching $k_{\max }$, differences are to be expected since the effective slope of the distribution function changes, becoming much steeper, until finally the number of optically thick lines vanishes and $\hat{\alpha} \rightarrow 0$, as discussed below Eq. (22). Actually, this is what happens for realistic line distributions (cf. Fig. A1 and Sect. 4.2.8). In so far, this exercise provides some analytic understanding with regard to the behaviour of the effective $\hat{\alpha}$ value if either $k_{1}$ grows due to decreasing density or $k_{\max }$ decreases due to decreasing metallicity (cf. also Sect. 5).

If we calculate the optically thick line-force from (B1) by using a lower integration limit of $k_{1}$ as in (11), we obtain

$\hat{\alpha}\left(k^{\prime}\right) \approx \frac{g_{\mathrm{rad}}^{\mathrm{thick}}}{g_{\mathrm{rad}}^{\mathrm{tot}}}=$

$\frac{\left(1-\mathrm{e}^{-1}\right) \mathrm{e}^{-k^{\prime}}+\left(1+k^{\prime}\right)^{1-a} \Gamma\left(a, 1+k^{\prime}\right)-k^{1-a} \Gamma\left(a, k^{\prime}\right)}{\left(\left(1+k^{\prime}\right)^{1-a}-k^{\prime-a}\right) \Gamma(a)}$.

Note, that in this example $\hat{\alpha}$ depends only on the value of $k^{\prime}=k_{1} / k_{\max }$ and the power-law index $a$, however not on the individual variables $k_{1}$ and $k_{\max } . \Gamma(a, x)$ is here related to the incomplete Gamma function via $\Gamma(a, x)=$

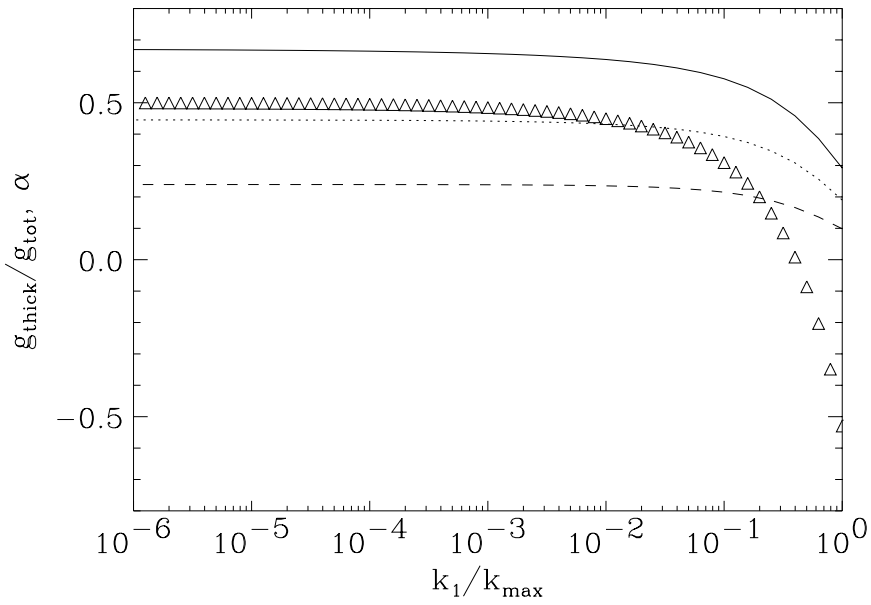

Fig. B1. Ratio of optically thick to total line-force $(\approx \hat{\alpha})$ as function of $k_{1} / k_{\max }$ (cf. Eq. B3) and $a=0.7,0.5,0.3$ (fully drawn, dotted, dashed), for the line-strength distribution function with exponential cutoff, Eq. (B1). The triangles display the local $\alpha$-values derived from the line-distribution function itself, corresponding to the case of $a=0.5$. Note, that $\alpha$ (local) $<\hat{\alpha}$ as soon as (A2) becomes invalid. The small deviations between acceleration ratio and $\hat{\alpha}$ for small $k_{1} / k_{\max }$ are due to our approximation leading to Eq. (28)

$\Gamma(a)-\gamma(a, x)$. In Fig. B1, we have plotted $\hat{\alpha}$ as function of $k^{\prime}$ for three values of $a=0.7,0.5$ and 0.3. Obviously, for $k_{1} \rightarrow k_{\max }$ (or vice versa) the effective $\hat{\alpha}$ becomes considerably smaller than the "input"-value, in agreement with our findings from Sect. 2.3.2. Note, however, that $\hat{\alpha}>0$ always and especially that it is much larger than the local $\alpha$ of the line-strength distribution function near $k_{\text {max }}$ !

\section{Appendix C: On the difference of $\bar{Q}$ and $Q_{\mathrm{o}}$}

In this appendix, we want to discuss the relation between $\bar{Q}$ and Gayley's cut-off parameter $Q_{\mathrm{o}}=v_{\mathrm{th}} / c k_{\max }$. In particular and related to the discussion in Sect. 3.3, the inequality $Q_{\mathrm{o}}>\bar{Q}$ which has been found for cooler atmospheres shall be inspected.

Using the definition of $\bar{Q}$ and the power-law linestrength distribution with exponential cutoff, Eq. (B1), $\bar{Q}$ can be expressed as

$$
\begin{aligned}
\bar{Q} & =\frac{v_{\mathrm{th}}}{c} \sum \frac{L_{\nu_{\mathrm{i}}} \nu_{\mathrm{i}}}{L} k_{\mathrm{L}}^{\mathrm{i}} \\
& \rightarrow N_{\mathrm{o}}\left\langle\frac{L_{\nu} \nu}{L}\right\rangle \frac{v_{\mathrm{th}}}{c} \int_{0}^{\infty} k_{\mathrm{L}}^{\alpha-1} \exp \left(-k_{\mathrm{L}} / k_{\max }\right) \mathrm{d} k_{\mathrm{L}}= \\
& =N_{\mathrm{o}}\left\langle\frac{L_{\nu} \nu}{L}\right\rangle \frac{v_{\mathrm{th}}}{c} \Gamma(\alpha) k_{\text {max }}^{\alpha}
\end{aligned}
$$

where $\left\langle\frac{L_{\nu} \nu}{L}\right\rangle$ is an appropriate average of the weight factor, e.g.,

$\left\langle\frac{L_{\nu} \nu}{L}\right\rangle=\frac{\sum \frac{L_{\nu_{\mathrm{i}}} \nu_{\mathrm{i}}}{L} k_{\mathrm{L}}^{\mathrm{i}}}{\sum k_{\mathrm{L}}^{\mathrm{i}}}$ 
Note, that both $\bar{Q}$ and the average weight factor depend essentially on the corresponding values for the strongest lines, due to the linear dependence on $k_{\mathrm{L}}$, a behaviour which was already addressed in Gayley's (1995) paper. In the previous expression, we have implicitely assumed that curvature effects concerning $\alpha$ are unimportant, i.e., that $\hat{\alpha} \approx \alpha$ everywhere except at highest line-strengths.

On the other hand, the flux (times frequency) weighted cumulative line number, evaluted at maximum line-strength, gives

$$
\begin{aligned}
& \left.\left\langle N\left(k_{\max }\right)\right\rangle \approx\left\langle\frac{L_{\nu} \nu}{L}\right\rangle\right|_{k_{\max }} \approx \\
& \left.\approx N_{\mathrm{o}}\left\langle\frac{L_{\nu} \nu}{L}\right\rangle\right|_{k_{\max }} \int_{k_{\max }}^{\infty} k_{\mathrm{L}}^{\alpha-2} \exp \left(-k_{\mathrm{L}} / k_{\max }\right) \mathrm{d} k_{\mathrm{L}}= \\
& =\left.N_{\mathrm{o}}\left\langle\frac{L_{\nu} \nu}{L}\right\rangle\right|_{k_{\max }} \Gamma(\alpha-1,1) k_{\max }^{\alpha-1}= \\
& =\left.N_{\mathrm{o}}\left\langle\frac{L_{\nu} \nu}{L}\right\rangle\right|_{k_{\max }} \Gamma(\alpha-1,1) k_{\max }^{\alpha}\left(\frac{c}{v_{\mathrm{th}}} Q_{\mathrm{o}}\right)^{-1}
\end{aligned}
$$

where in the rhs of the first line this number (without weight!) has been set to unity (i.e., $Q_{\mathrm{o}}$ shall be the linestrength of the single strongest line) and the subscript " $k_{\max }$ " accounts for the weighting process at the according frequency. By comparing the above two expressions, we obtain for the flux-weighted number of this single strongest line

$$
\begin{aligned}
\left\langle N\left(k_{\max }\right)\right\rangle & =\frac{\left.\left\langle\frac{L_{\nu} \nu}{L}\right\rangle\right|_{k_{\max }} \bar{Q} \Gamma(\alpha-1,1)}{\left\langle\frac{L_{\nu} \nu}{L}\right\rangle Q_{\mathrm{o}} \Gamma(\alpha)}= \\
& =\frac{\left.\left\langle\frac{L_{\nu} \nu}{L}\right\rangle\right|_{k_{\max }}}{\left\langle\frac{L_{\nu} \nu}{L}\right\rangle} \frac{\bar{Q}}{Q_{\mathrm{o}}} \frac{\left(\mathrm{e}^{-1}-\Gamma(\alpha, 1)\right)}{(1-\alpha) \Gamma(\alpha)},
\end{aligned}
$$

which can be inverted (again by requiring $N\left(k_{\max }\right)=1$ ) to yield the corresponding line-strength

$Q_{\mathrm{o}} \approx\left\langle\frac{L_{\nu} \nu}{L}\right\rangle^{-1} \bar{Q} f(\alpha)$.

The last factor in this equation $(=\Gamma(\alpha-1,1) / \Gamma(\alpha))$ has a value of $0.14,0.10$ and 0.077 for typical $\alpha$ 's $=0.66,0.5$ and 0.4 .

Thus, in cases where the frequential line-distribution is essentially independent on line-strength and is distributed according to CAK's assumption, $\mathrm{d} N \propto \mathrm{d} \nu / \nu$ and hence $\left\langle\frac{L_{\nu} \nu}{L}\right\rangle=1, Q_{\mathrm{o}}$ should be (slightly) smaller than $\bar{Q}$, in contrast to what is "observed" especially for cooler atmospheres. Vice versa, by accounting for the actual similarity of $\bar{Q}$ and $Q_{\text {o }}$ in hotter atmospheres (Table 2), one might argue that the "effective" number of lines with strength $Q_{\mathrm{o}}$ shall be of order $f(\alpha)$. Independently from these more "philosophical" questions (involving uncertainties of order one dex), the plain fact that the ratio $Q_{\mathrm{o}} / \bar{Q}$ is larger than unity and increasing for decreasing $T_{\text {eff }}$ inevitably leads to the conclusion that the average factor has to be significantly below unity and is decreasing in parallel with $T_{\text {eff }}$. Both by exploring the frequential line-distribution of the strongest lines (cf. Fig. 4 and the accompanying text) as well as by simply calculating the average weight factor (Eq. C2), it turns out that this is actually the case: for the same model atmospheres as in Sect. 3.2, we find $\left\langle\frac{L_{\nu} \nu}{L}\right\rangle=0.4$ for $T_{\text {eff }}=50000 \mathrm{~K}$ and $\left\langle\frac{L_{\nu} \nu}{L}\right\rangle=0.003$ for $T_{\text {eff }}=10000 \mathrm{~K}$. Inserting these values and using the derived $\alpha$-values from Table $2, Q_{\mathrm{o}} / \bar{Q}=0.35$ and 28 are predicted by means of (C5). The differences to the actual values of 1.16 and 16 , respectively, remain to be attributed to the deviations from a perfect power-law, especially at the predominantely contributing high $k_{\mathrm{L}}$-end of the distribution.

\section{Appendix D: LTE line-strength distribution for hydrogenic ions}

In LTE and using Kramer's formula, the hydrogen linestrength reads

$$
\begin{aligned}
& k_{\mathrm{L}}=\frac{\bar{\chi}_{\mathrm{i}} \lambda_{\mathrm{i}}}{\rho} \frac{1}{s_{\mathrm{E}} v_{\mathrm{th}}} \propto \frac{\lambda_{\mathrm{i}}}{\rho} g f\left(\frac{n_{l}}{g_{l}}-\frac{n_{u}}{g_{u}}\right) \\
& \propto \frac{n_{1}}{\rho}\left(\frac{1}{n^{\prime 2}}-\frac{1}{n^{2}}\right)^{-4}\left(\frac{1}{n^{3} n^{\prime 3}}\right)\left(\mathrm{e}^{-h \nu_{l} / k T_{\mathrm{e}}}-\mathrm{e}^{-h \nu_{u} / k T_{\mathrm{e}}}\right),
\end{aligned}
$$

where $n_{1}$ is the ground-state occupation number, $h \nu_{l}, h \nu_{u}$ are the excitation energies of the lower and upper level, and the dependence on the transition wavelength $\lambda_{\mathrm{i}}$ transforms into an additional power of the second factor. Realizing that the appropriate variable for a further expansion is given by $x=\left(n / n^{\prime}\right)^{3}<1$, and denoting

$\tilde{k}\left(n^{\prime}, T_{\mathrm{e}}\right)=\frac{k_{\mathrm{L}} \exp \left(\frac{h \nu_{1}}{k T_{\mathrm{e}}}\right)}{C^{\prime} n^{\prime 2}}$

with $h \nu_{1}$ the ground-state ionization energy and $C^{\prime}$ a factor absorbing all "constants" (most important: $n_{1} / \rho$ ) in the equation before, we can write

$$
\begin{aligned}
\tilde{k}\left(n, n^{\prime}, T_{\mathrm{e}}\right) & =\frac{x}{\left(1-x^{2 / 3}\right)^{4}}\left(K\left(T_{\mathrm{e}}, n^{\prime}\right)-K\left(T_{\mathrm{e}}, n^{\prime}\right)^{x^{2 / 3}}\right) \\
K\left(T_{\mathrm{e}}, n^{\prime}\right) & =\exp \left(\frac{h \nu_{1}}{k T_{\mathrm{e}} n^{\prime 2}}\right)
\end{aligned}
$$

This equation can be expanded in powers of $x$, inverted and solved for $n$ :

$$
\begin{aligned}
x\left(\tilde{k}, n^{\prime}, T_{\mathrm{e}}\right) & =\frac{\tilde{k}}{K-1}-\left(\frac{1}{K-1}\right)^{8 / 3} \times \\
& \times[4(K-1)-\ln (K)] \tilde{k}^{5 / 3}+O(\tilde{k})^{7 / 3} \\
n\left(k_{\mathrm{L}}, n^{\prime}, T_{\mathrm{e}}\right) & =n^{\prime} x^{-1 / 3}=n^{\prime}\left(\frac{K-1}{\tilde{k}}\right)^{\frac{1}{3}}+O(\tilde{k})^{1 / 3} .
\end{aligned}
$$

This expression is valid for not too large line-strengths $k_{\mathrm{L}}<C^{\prime} \exp \left(-h \nu_{1} / k T_{\mathrm{e}}\right)$. Summing up again the number of lines with line-strengths larger/equal than a given value, we finally obtain the result given in (50), 


$$
\begin{aligned}
& N\left(k_{\mathrm{L}}, T_{\mathrm{e}}\right)=\sum_{n^{\prime}=1}^{n_{\max }^{\prime}} n\left(k_{\mathrm{L}}, n^{\prime}, T_{\mathrm{e}}\right)-n^{\prime}= \\
= & k_{\mathrm{L}}^{-\frac{1}{3}}\left(C^{\prime} \mathrm{e}^{-\frac{h \nu_{1}}{k T_{\mathrm{e}}}}\right)^{\frac{1}{3}} f\left(T_{\mathrm{e}}\right)-\frac{n_{\max }^{\prime}\left(n_{\max }^{\prime}+1\right)}{2}, \\
& f\left(T_{\mathrm{e}}\right)=\sum_{n^{\prime}=1}^{n_{\max }^{\prime}} n^{\prime \frac{5}{3}}\left(\mathrm{e}^{\frac{h \nu_{1}}{k T_{\mathrm{e}} n^{\prime 2}}}-1\right)^{\frac{1}{3}} \text { and } k_{\mathrm{L}}<C^{\prime} \mathrm{e}^{-\frac{h \nu_{1}}{k T_{\mathrm{e}}}} .
\end{aligned}
$$

\section{Appendix E: Frequency-integration of the line intensity distribution function}

In this appendix, we give a brief derivation of the frequency integrated line intensity distribution function, Eq. (73). Similar to the frequency dependent one, Eq. (61), which is the starting point of our considerations, we have to account for different regimes, since the maximum possible (logarithmic) oscillator strength $\tilde{r}_{\max }$ depends both on line intensity and frequency. Furthermore, we assume that under certain circumstances only levels below a cutoff $x_{\max }$ shall contribute. In this case then, all lines with lower levels energetically higher than $x_{\max }$ are neglected. This generalization will turn out to be important if NLTE-effects are included into our approach (cf. Sect. 4.2.3).

Finally, we allow for an integration between $0<\nu<$ $\nu_{\max } \leq i_{\mathrm{e}}$, since for high ionization energies our line list may be incomplete (and useless, if one accounts for the vanishing flux) beyond a certain maximum frequency.

At first note, that the maximum possible oscillator strength is given by

$\tilde{r}_{\max }=\operatorname{Min}\left(\frac{i_{\mathrm{e}}-\nu}{t}-l,-l_{\min }\right)$,

in accordance with Eq. (64). Accounting additionally for a possible incompleteness of the level list, $x_{1} \leq x_{\max }$, and noting that the minimum value for $r$ is given by $r_{\text {min }}$, we have to extend this restriction further:

$\tilde{r}_{\max }=\operatorname{Max}\left(\operatorname{Min}\left(\frac{i_{\mathrm{e}}-\nu}{t}-l, \frac{x_{\max }}{t}-l,-l_{\min }\right), r_{\min }\right)$.

From this expression, we can derive the maximum frequency until which the integration has to be performed,

$\nu_{\text {up }}=\operatorname{Min}\left(\nu_{\max }, \nu_{r}=i_{\mathrm{e}}-t\left(l+r_{\min }\right)\right)$,

since for frequencies larger than $\nu_{r}$ we have $\tilde{r}_{\max }=r_{\min }$ and the according number of lines is zero (cf. Eq. 61). Furthermore, the allowed range of $l$ is given by

$l_{\min } \leq l \leq l_{\max }=\frac{x_{\max }}{t}-r_{\min }$,

where two subranges have to be defined in order to satisfy Eq. (E1):

At low line intensities, $l<x_{\max } / t+l_{\min }$, we have to account for the minimum $\operatorname{Min}\left(\left(i_{\mathrm{e}}-\nu\right) / t-l,-l_{\min }\right)$, which introduces a threshold frequency $\nu_{l}=i_{\mathrm{e}}-t\left(l-l_{\min }\right)<\nu_{r}$ :

$l<\frac{x_{\max }}{t}+l_{\min }$ : $\tilde{r}_{\max }=\left\{\begin{array}{ll}-l_{\min } & \text { for } \nu_{\max }<\nu_{l} \\ \frac{i_{\mathrm{e}}-\nu}{t}-l & \text { for } \nu_{\mathrm{up}}>\nu>\nu_{l} . \\ -l_{\min } & \text { for } \nu_{l}>\nu>0\end{array}\right.$.

In the second regime with $x_{\max } / t-l<-l_{\min }$, the maximum oscillator strength $-l_{\min }$ cannot be reached any longer, and the appropriate threshold frequency $\nu_{x}=$ $i_{\mathrm{e}}-x_{\max }$ introduces the following possibilities:

$\frac{x_{\max }}{t}+l_{\min }<l<l_{\max }$ :

$\tilde{r}_{\max }=\left\{\begin{array}{ll}\frac{x_{\max }}{t}-l & \text { for } \nu_{\max }<\nu_{x} \\ \frac{i_{\mathrm{e}}-\nu}{t}-l & \text { for } \nu_{\mathrm{up}}>\nu>\nu_{x} \\ \frac{x_{\max }}{t}-l & \text { for } \nu_{x}>\nu>0\end{array}\right.$,

since $\nu_{r}>\nu_{x}$ always. $\left(\nu_{r}=\nu_{x}\right.$ is reached only at $l=l_{\max }$.)

By integrating Eq. (61) over frequency and accounting for these different cases (four in total), we finally obtain the following expressions for the function $F$ defined in Eq. (73).

$$
\begin{aligned}
& F \quad\left(l, t, \sigma, i_{\mathrm{e}}, x_{\max }, \nu_{\max }, \gamma, l_{\min }, r_{\min }\right)= \\
&=\left(10^{-A l_{\min }}-10^{A r_{\min }}\right)\left(10^{\frac{\nu_{\max }}{\sigma}}-1\right), \\
&\left(l<\frac{x_{\max }}{t}+l_{\min }, \nu_{\max }<\nu_{l}\right) . \\
&= \frac{10\left(\frac{i_{\mathrm{e}}}{t}-l\right)}{1-\frac{A \sigma}{t}}\left(10^{\frac{\nu_{\mathrm{up}}}{\sigma}\left(1-\frac{A \sigma}{t}\right)}-10^{\left.\frac{\nu_{l}}{\sigma}\left(1-\frac{A \sigma}{t}\right)\right)+}\right. \\
&+ 10^{-A l_{\min }}\left(10^{\frac{\nu_{l}}{\sigma}}-1\right)-10^{A r_{\min }}\left(10^{\frac{\nu_{\mathrm{up}}}{\sigma}}-1\right), \\
&\left(l<\frac{x_{\max }}{t}+l_{\min }, \nu_{\max }>\nu_{l}\right) .
\end{aligned}
$$

$$
\begin{aligned}
= & \left(10^{A\left(\frac{x_{\max }}{t}-l\right)}-10^{A r_{\min }}\right)\left(10^{\frac{\nu_{\max }}{\sigma}}-1\right), \\
& \left(l<l_{\max }, \nu_{\max }<\nu_{x}\right) . \\
= & \frac{10\left(\frac{i_{\mathrm{e}}}{t}-l\right)}{1-\frac{A \sigma}{t}}\left(10^{\frac{\nu_{\mathrm{up}}}{\sigma}\left(1-\frac{A \sigma}{t}\right)}-10^{\left.\frac{\nu_{x}}{\sigma}\left(1-\frac{A \sigma}{t}\right)\right)}\right. \\
+ & A\left(\frac{x_{\max }}{t}-l\right)\left(10^{\frac{\nu_{x}}{\sigma}}-1\right)-10^{A r_{\min }}\left(10^{\frac{\nu_{\mathrm{up}}}{\sigma}}-1\right), \\
& \left(l<l_{\max }, \nu_{\max }>\nu_{x}\right) .
\end{aligned}
$$

For convenience, we have summarize the required threshold values below:

$$
\begin{aligned}
\nu_{\mathrm{up}} & =\operatorname{Min}\left(\nu_{\max }, \nu_{r}\right) \\
\nu_{r} & =i_{\mathrm{e}}-t\left(l+r_{\min }\right) \\
\nu_{l} & =i_{\mathrm{e}}-t\left(l-l_{\min }\right) \\
\nu_{x} & =i_{\mathrm{e}}-x_{\max } \\
l_{\max } & =\frac{x_{\max }}{t}-r_{\min }
\end{aligned}
$$


Note that due to the specific combinations of energies, frequencies and temperature derived above, the function $F$ depends not independently on any of the variables $\left(t, \sigma, i_{\mathrm{e}}, x_{\max }, \nu_{\max }\right)$, however only on certain ratios: The line intensity distribution function remains completely unchanged if the above variable set preserves its ratio with respect to either $t, \sigma$ or $i_{\mathrm{e}}$. In the following, we will mostly consider the normalization with respect to $\sigma$, i.e., understand $F$ as function of $\left(t / \sigma, i_{\mathrm{e}} / \sigma, x_{\max } / \sigma\right.$ and $\left.\nu_{\max } / \sigma\right)$.

The most important fact concerning the derived function is the following: Depending on line intensity and the specific value of $A$, the distribution can show three different slopes, namely $2 t / \sigma, t / \sigma$ and $\gamma-1$, where the first one and the last have been found already for the frequency dependent distribution function (cf. Sect. 4.2.1), whereas the second one is a new feature arising from the frequency integration.

Let us briefly consider under which condition which slope will show up. At first, assume that $x_{\max }=\nu_{\max }=i_{\mathrm{e}}$, i.e., both the level list and the line list shall be complete. In this case, Eqs. (E7) and (E9) have to be applied $\left(\nu_{x}=0\right)$.

For (very) low line intensities, $l$ is approximately $l_{\text {min }}$ and $\nu_{l} \approx i_{\mathrm{e}}=\nu_{\mathrm{up}}$. Thus, the second term in (E7) dominates and the result is similar to (E6), with maximum frequency $i_{\mathrm{e}}$. In consequence, $F$ is independent on $l$ and the apparent slope of the distribution is $2 t / \sigma$ (remember, that $\left.\Delta N(l) \propto 10^{2 l t / \sigma} F\right)$. In this situation, for (almost) all possible line frequencies the contributing oscillator strengths stretch from $r_{\min }$ to $r_{\max }=-l_{\min }$.

For larger $l$ then, $\nu_{l}$ decreases, whereas $\nu_{\mathrm{up}}=i_{\mathrm{e}}$ for $l$ being smaller than $-r_{\text {min }}$. We encounter the case that the maximum possible oscillator strength $-l_{\min }$ can no longer be reached for large frequencies (cf. Eq. (E4), middle panel), and the apparent slope is controlled by the sign of $A$. For negative and not too small $A$, i.e., $\gamma>\gamma_{\text {crit }}$,

$\gamma_{\text {crit }}=1+2 t / \sigma$

the lower end of the oscillator strength distribution dominates by far, and $F$ depends solely on the last term of (E7). In consequence, $\Delta N$ retains its slope of $2 t / \sigma$.

For positive $A$, i.e., $\gamma<\gamma_{\text {crit }}$, the situation is different. Now the first bracket of (E7) dominates, giving rise (via the combination of exponents $2 l t / \sigma+A\left(i_{\mathrm{e}} / t-l\right)+$ $\left.\nu_{l} / \sigma(1-A \sigma / t)\right)$ to an exact slope of $t / \sigma$, since the dependence on $\gamma$ cancels completely. This behaviour is finally reached also for negative $A$ and larger $l$ : For $l>-r_{\min }$, the upper frequential boundary $\nu_{\text {up }}$ is $\nu_{r}$, which has the same dependency on $l$ as $\nu_{l}$. Additionally, the impact of small oscillator strengths becomes smaller, simply because the last term in (E6) decreases with $\nu_{r}$ as function of $l$.

Nothing changes for negative $A$ and even larger $l$, when Eq. (E9) applies. The slope remains at its value $t / \sigma$. For positive $A$, however, there is a dramatic change for $l>i_{\mathrm{e}} / t+l_{\min }$. Due to the interrelation of line intensity and $\tilde{r}_{\max }$ (cf. the discussion in Sect. 4.2.1), the dependence on $t / \sigma$ cancels and only the $\gamma-1$ slope survives (the 2 nd

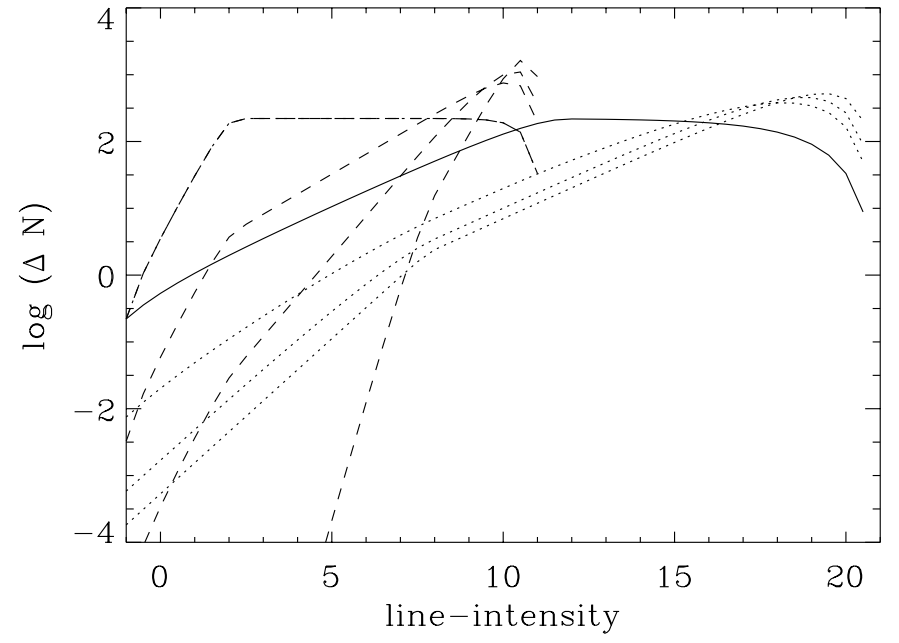

Fig. E1. Frequency integrated line intensity distribution function: variation with $\gamma$ and $t / \sigma$. Basic parameters similar to the case of NIV, $i_{\mathrm{e}} / \sigma=3.0, \nu_{\max }=x_{\max }=i_{\mathrm{e}}=620 \mathrm{kK}$, $l_{\min }=-1, r_{\min }=-8$ and $\log N\left(l_{\max }\right)=3.6$. Fully drawn: $\gamma=1.0, t / \sigma=0.23$ (corresp. to $T=30000 \mathrm{~K}$ ); dotted: $\gamma=1.3,1.6,3.0, t / \sigma=0.23$. Dashed-dotted: $\gamma=1.0, t / \sigma=0.93$ (corresponding to $T=120000 \mathrm{~K}$ ); dashed: $\gamma=1.3,1.6,3.0, t / \sigma=0.93$

term in (E9) being now the dominating one). Thus for $\gamma$ (well) below $\gamma_{\text {crit }}$ the apparent slope at high line intensities is coupled to the oscillator strength statistics. Finally if $\gamma \approx \gamma_{\text {crit }}$ (corresponding to the case $A=0$ which cannot be treated by the above formalism), it turns out that the slope at large $l$ smoothly changes from $(\gamma-1)$ to $t / \sigma$.

In summary, we have the following behaviour of $\Delta N$ if $x_{\max }=\nu_{\max }=i_{\mathrm{e}}$ :

$$
\begin{gathered}
\gamma>\gamma_{\text {crit }}: \quad \log \Delta N \propto \begin{cases}\frac{2 t}{\sigma} l & \text { for } l<-r_{\text {min }} \\
\frac{t}{\sigma} l & \text { for } l>-r_{\text {min }}\end{cases} \\
\gamma \lesssim \gamma_{\text {crit }}: \log \Delta N \propto\left\{\begin{array}{cl}
\frac{2 t}{\sigma} l & \text { for } l \approx l_{\text {min }} \\
\frac{t}{\sigma} l & \text { for } l<\frac{i_{\mathrm{e}}}{t}+l_{\text {min }} \\
\text { Min } & \left((\gamma-1), \frac{t}{\sigma}\right) l \\
& \text { for } l>\frac{i_{\mathrm{e}}}{t}+l_{\text {min }} .
\end{array}\right.
\end{gathered}
$$

Figure E1 illustrates the described behaviour by means of the line intensity distribution function of NIV, normalized to $\log N\left(l_{\max }\right)=3.6$. Note that the ordinate stretches to (unphysical) negative values, in order to display the changes of apparent slope as function of $l$.

We have considered two case, namely $t / \sigma=0.23$ and 0.93 , respectively, to demonstrate the dependence on $\gamma_{\text {crit }}$. In the first case then, $\gamma_{\text {crit }}=1.46$, and the fully drawn line $(\gamma=1.0)$ and the dotted ones $\gamma=1.3,1.6,3.0$ display the resulting distribution functions. As predicted by Eq. (E12), the curve for $\gamma=1.0$ displays two effective slopes, namely $t / \sigma$ and $(\gamma-1)$, where the dividing line intensity is given by $i_{\mathrm{e}} / t+l_{\text {min }}=12$. For $\gamma=1.3$, only one slope $(t / \sigma)$ is present due to the third condition in 


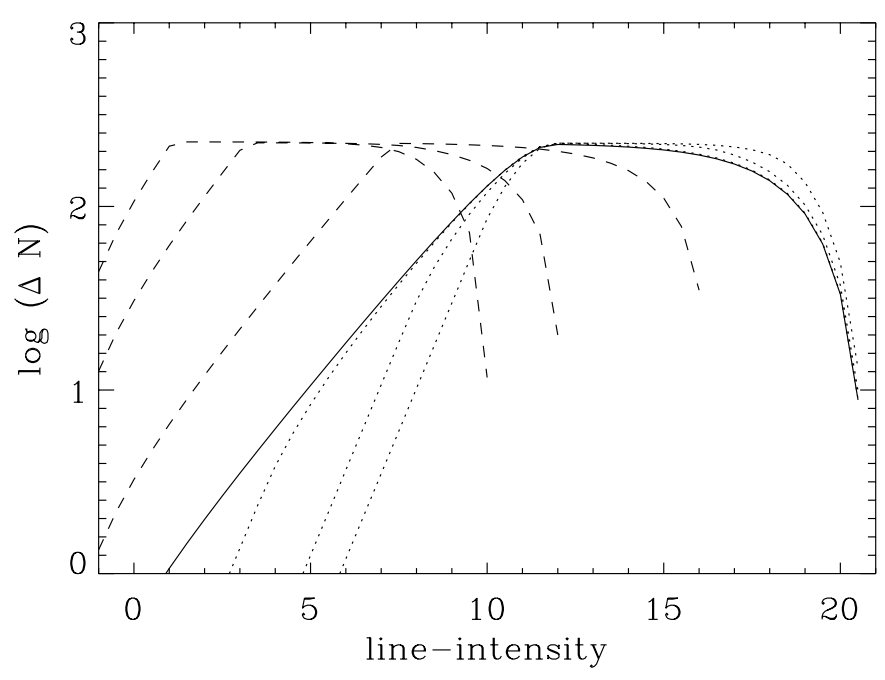

Fig. E2. Frequency integrated line intensity distribution function: variation with $\nu_{\max }$ and $x_{\max }$. Basic parameters as in Fig. E1 with $T=30000 \mathrm{~K}$ and $\gamma=1.0$. Fully drawn: $x_{\max }=\nu_{\max }=i_{\mathrm{e}} ;$ dotted: $x_{\max }=i_{\mathrm{e}}, \nu_{\max }=400,200,100 \mathrm{kK}$; dashed: $\nu_{\max }=i_{\mathrm{e}}, x_{\max }=400,200,100 \mathrm{kK}$

Eq. (E12). In contrast, the behaviour for $\gamma=1.6$ and 3 $\left(>\gamma_{\text {crit }}\right.$, Eq. $($ E11) $)$ depends on $2 t / \sigma$ and $t / \sigma$, with a corresponding boundary at $l=-r_{\min }=8$ in our example.

For the larger value of $t / \sigma$ with $\gamma_{\text {crit }}=2.86$, we see the transition of slope $t / \sigma$ to $\gamma-1$ at $l=i_{\mathrm{e}} / t+l_{\min }=2$ for the curves with $\gamma=1.0$ (dashed-dotted), $\gamma=1.3$ and 1.6 (dashed). Only the case with $\gamma=3>\gamma_{\text {crit }}$ reaches its asymptotic value of $t / \sigma \approx 1.9$, where also the steeper slope of $2 t / \sigma$ for $l<-r_{\min }$ is clearly visible.

In Fig. E2 we demonstrate the influence of either varying the highest energetic level $\left(x_{\max }\right)$ or the maximum line frequency $\left(\nu_{\max }\right)$, if all other parameters are kept constant. As long as $x_{\max }=i_{\mathrm{e}}$ (dotted curves), the only influence of decreasing $\nu_{\max }$ concerns the region with $l<i_{\mathrm{e}} / t+l_{\min }$, i.e., Eq. (E6) (partly) replaces Eq. (E7), where the largest influence is close to $l_{\min }$. Only for very small values of $\nu_{\max }$ the complete first interval is affected. In consequence, even for $\gamma<\gamma_{\text {crit }}$ the apparent slope becomes $2 t / \sigma$, since $F$ depends no longer on $l$.

In contrast, by diminishing $x_{\max }$ while keeping $\nu_{\max }=$ $i_{\mathrm{e}}$ (dashed curves), the slope of the first interval is barely affected, unless $x_{\max }$ becomes (very) small compared to $i_{\mathrm{e}}$ so that the transition value is close to $l_{\text {min }}$ (cf. E12, first panel). The major effect of decreasing $x_{\max }$, however, is by shifting the dividing line (which is now a linear function of $\left.x_{\max }\right)$ to smaller line intensities. Thus, a line-distribution with $x_{\max }$ different from $i_{\mathrm{e}}$ resembles the line-distribution of a similar ion, however with much smaller ionization energy. This behaviour turns out to be important if one considers the NLTE line-strength statistics. Finally, if $x_{\max }$ approaches zero, the line distribution becomes independent on any excitation effects. This limiting case, which corresponds to accounting for resonance lines only, leads

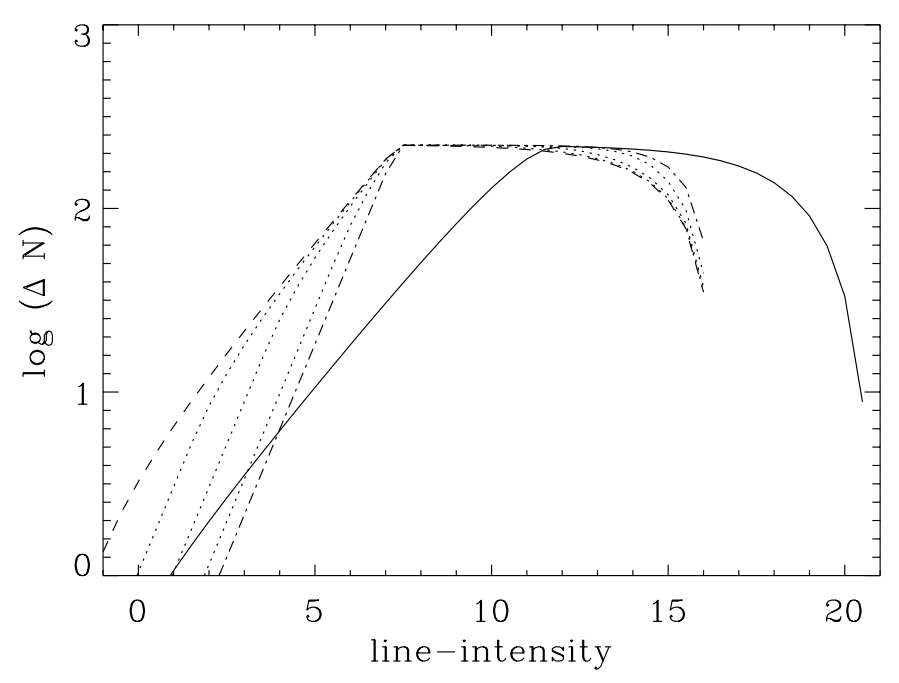

Fig. E3. Frequency integrated line intensity distribution function: saturation effect. Basic parameters as in Fig. E2. Fully drawn: $x_{\max }=\nu_{\max }=i_{\mathrm{e}}$; dashed: $x_{\max }=400 \mathrm{kK}, \nu_{\max }=i_{\mathrm{e}}$; dotted: $x_{\max }=400 \mathrm{kK}, \nu_{\max }=500,400,300 \mathrm{kK}$; dasheddotted: "saturation" limit for $\nu_{\max } \leq 220 \mathrm{kK}$

to a line-statistics influenced solely by the underlying $g f$-distribution.

Figure E3 illustrates the effect of diminishing both $x_{\text {max }}$ and $\nu_{\max }$. In principle, the effects are similar to the cases studied above, namely the transition value is changed via $x_{\max }$, and the slope of the first region increases to $2 t / \sigma$. However, there exists another interesting effect, displayed by the dashed dotted curve: If the maximum considered frequency $\nu_{\max }$ falls below the value of $\nu_{x}=i_{\mathrm{e}}-x_{\max }$, the distribution function becomes "saturated", i.e., does no longer change in shape (of course, the absolute value of $F$ and thus the total line number decreases with $\nu_{\max }$ ). The reason for this saturation is given by the fact that Eqs. (E6) and (E8) now control the behaviour of $F$, and that for $\nu_{\max }<\nu_{x}$ this function depends on $\nu_{\max }$ solely by a constant factor $\left(10^{\nu_{\max } / \sigma}-1\right)$ for all $l$.

Note, that in those cases when $x_{\max }$ is small compared to $i_{\mathrm{e}}$ (as is typical under NLTE-conditions, see Sect. 4.2.3), this condition applies for fairly large $\nu_{\max }$. In other words: The shape of the function is the same for all cutoff frequencies smaller than $i_{\mathrm{e}}-x_{\max }$ : At maximum two slopes are present, namely either $2 t / \sigma$ and $\gamma-1$ for $\gamma<\gamma_{\text {crit }}$ or $2 t / \sigma$ for $\gamma>\gamma_{\text {crit }}$. This fact is essential for flux-weighted line-strength distribution function (Sect. 4.2.8), since the maximum frequency which has to be considered for this function is fairly small due to the decreasing flux at high energies.

Acknowledgements. We like to thank Ken Gayley, Stan Owocki and Achim Feldmeier for useful comments and suggestions. This project has been supported in part by the Deutsche Forschungsgemeinschaft under DFG grants $\mathrm{Pu} 117-1 / 2$ and $\mathrm{Pa}$ $477-1 / 2$. 


\section{References}

Abbott D.C., 1980, ApJ 242, 1183

Abbott D.C., 1982, ApJ 259, 282

Abbott D.C., Lucy L.B., 1985, ApJ 288, 679

Allen C.W., 1966, MNRAS 133, 21

Allen C.W., 1974, MNRAS 168, 121

Arav N., Li Z., 1994, ApJ 427, 700

Arav N., Li Z., Begelman M.C., 1994, ApJ 432, 62

Bjorkman J.E., Cassinelli J.P., 1993, ApJ 409, 429

Castor J.I., Abbott D.C., Klein R.I., 1975, ApJ 195, 157 (CAK)

Feldmeier A., Norman C., Pauldrach A., et al., 1997, in: Mass ejection from AGNs, PASP Conf. Ser., Arav N., Shlosman I. \& Weyman R. (eds.) (in press)

Friend D.B., Abbott D.C., 1986, ApJ 311, 701

Garmany C.D., Conti P.S., 1984, ApJ 284, 705

Gayley K., 1995, ApJ 454, 410

Haser S.M., Lennon D.J., Kudritzki R.P., et al., 1994, Sp. Sci. Rev. 66, 179

Haser S.M., 1995, Thesis, Ludwig-Maximilians-Universität München

Howarth J.D., Prinja R.K., 1989, ApJS 69, 527

Kudritzki R.P., 1997, in: Aparicio A., Herrero, A. (eds.) 8th Canary Winter School, Stellar Astrophysics for the Local Group. MPA-Sonderdruck 1023, Garching, p. 1

Kudritzki R.P., Pauldrach A., Puls J., 1987, A\&A 173, 293

Kudritzki R.P., Pauldrach A., Puls J., Abbott D.C., 1989, A\&A 219, 205

Kudritzki R.P., Lennon D.J., Puls J., 1995, in: Science with the Very Large Telescope, Walsh J.R. \& Danziger I.J. (eds.). ESO Astrophysics Symposia, Springer, Heidelberg, p. 246

Kudritzki R.P., Springmann U., Puls J., Pauldrach A.W.A., Lennon M., 1998, PASPC 131, 299

Kudritzki R.P., Puls J., Lennon D.J., et al., 1999, A\&A (in press)

Kurucz R.L., 1995, PASPC 78, 205

Learner R.C.M., 1982, J. Phys. B: At. Mol. Phys. 15, L891

Lucy L.B., Solomon P., 1970, ApJ 159, 879

Lucy L.B., Abbott D.C., 1993, ApJ 405, 738

McCarthy J.K, Lennon D.J., Venn K.A., et al., 1995, ApJL 455, L135

Mihalas D., 1978, Stellar Atmospheres. 2nd Edition. Freeman,
San Francisco

Owocki S.P., Rybicki G.B., 1984, ApJ 284, 337

Owocki S.P., Rybicki G.B., 1985, ApJ 299, 265

Owocki S.P., Castor J.I., Rybicki G.B., 1988, ApJ 335, 914

Owocki S.P., Puls J., 1996, ApJ 462, 894

Owocki S.P., Cranmer S.R., Blondin J.M., 1994, ApJ 424, 887

Owocki S.P., Cranmer S.R., Gayley K.G., 1996, ApJ 472, L1150

Owocki S.P., Puls J., 1996, ApJ 510, 355

Owocki S.P., Gayley K.G., 1999, Proc. IAU Symp. 193, van der Hucht K.A., Koenigsberger G., Eeenens P.R.J. (eds.) (in press)

Pauldrach A.W.A., Puls J., Kudritzki R.P., 1986, A\&A 164, 86

Pauldrach A.W.A., 1987, A\&A 183, 295

Pauldrach A.W.A., Kudritzki R.P., Puls J., Butler K., 1990, A\&A 228, 125

Pauldrach A.W.A., Kudritzki R.P., Puls J., Butler K., Hunsinger J., 1994, A\&A 283, 525

Pauldrach A.W.A., Lennon M., Hoffmann T.L., et al., 1998, PASPC 131, 258

Petrenz P., 1999, Thesis, Ludwig-Maximilians-Universität München

Puls J., 1987, A\&A 184, 227

Puls J., Kudritzki R.P., Herrero A., et al., 1996, A\&A 305, 171

Schaerer D., Schmutz W., 1997, A\&A 321, 268

Schmutz W., 1991, in: Stellar Atmospheres: Beyond Classical Models, Crivellari L., Hubeny I., Hummer D.G. (eds.), NATO ASI Ser. C 341. Kluwer, Dordrecht, p. 191

Schmutz W., 1997, A\&A 288, 231

Seaton M.J., et al., 1995, The Opacity Project, Compiled by the Opacity Project Team. IUP, Bristol

Springmann U., 1997, Thesis, Ludwig-Maximilians-Universität München

Springmann U., Puls J., 1998, PASPC 131, 286

Vink J.S., de Koter A., Lamers H.J.G.L.M., 1999, A\&A (submitted)

Walborn N.R., Lennon D.J., Haser S.M., Kudritzki R.P., Voels S.A., 1995, PASP 107, 104

Wehrse R., von Waldenfels W., Baschek B., 1998, JQRST 60, 963 